\title{
Scope of the Asymmetric Intramolecular Stetter Reaction Catalyzed by Chiral Nucleophilic Triazolinylidene Carbenes
}

\author{
Javier Read de Alaniz, Mark S. Kerr, Jennifer L. Moore and Tomislav Rovis* \\ Department of Chemistry, Colorado State University \\ Fort Collins, Colorado 80523 \\ rovis@lamar.colostate.edu
}

\section{Supporting Information}

$\begin{array}{ll}\text { Table of Contents } & \text { S-1 }\end{array}$

$\begin{array}{ll}\text { General Considerations } & \text { S-2 }\end{array}$

Experimental Procedures for the Asymmetric Stetter Reaction $\quad$ S-2

$\begin{array}{ll}\text { NMR Spectra } & \text { S-22 }\end{array}$

$\begin{array}{lr}\text { Reference } & \text { S-53 }\end{array}$ 
General Methods. All reactions were carried out under atmosphere of argon in flamedried glassware with magnetic stirring. Visualization was accomplished with UV light, $\mathrm{KMnO}_{4}$, and aqueous ceric ammonium molybdate dips followed by heating.

Data for NMR are reported as follows: chemical shift in parts per million $(\delta \mathrm{ppm})$ from an internal standard [tetramethylsilane (TMS) or chloroform $\left(\mathrm{CHCl}_{3}\right)$ ], multiplicity $(\mathrm{s}=$ singlet, $\mathrm{d}=$ doublet, $\mathrm{t}=$ triplet, $\mathrm{q}=$ quartet, and $\mathrm{m}=$ multiplet $)$, integration, and coupling constant $(\mathrm{Hz}),{ }^{13} \mathrm{C}$ NMR chemical shifts are reported in ppm from $\mathrm{CDCl}_{3}$ (taken as $77.0 \mathrm{ppm}$ ). Analytical high performance liquid chromatography (HPLC) was performed using a Chiralcel OD-H, OB-H, AD-H, OJ, AS $(0.46 \mathrm{~cm} \mathrm{X} 25 \mathrm{~cm})$ chiral column. Gas Chromatography was performed using a Chiraldex B-DM and a CP Wax $52 \mathrm{CB}$ column.

Toluene was degassed with argon and passed through one column of neutral alumina and one column of Q5 reactant.

Starting material was prepared according to literature precedent. ${ }^{1}$ All spectral data was matched with literature

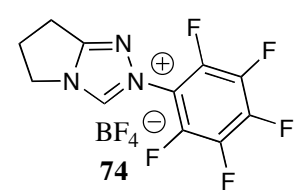
description. All racemic products were prepared analogously to the asymmetric reaction utilizing achiral catalyst 74. Chiral triazolium salts 4, 5, 6, 7, 45 and 74 were prepared according to our previously reported procedure. ${ }^{2}$ Compounds $9,11,15,17,19,21,23,29$ and $\mathbf{3 1}$ have been previously characterized. ${ }^{3,4,5}$

General procedure for the asymmetric intramolecular Stetter reaction: A flame dried round bottom flask was charged with triazolium salt $(0.2 \mathrm{eq})$ and $5 \mathrm{~mL}$ of toluene. To this solution was added KHMDS ( $0.5 \mathrm{M}$ in toluene prepared prior to use from $0.05 \mathrm{~g}$ of KHMDS in $0.5 \mathrm{~mL}$ of toluene) (0.2 eq) via syringe and the solution was stirred at 
ambient temperature for 5 minutes. A solution of the substrate ( $1 \mathrm{eq}, 0.12 \mathrm{mmol})$ in $2 \mathrm{~mL}$ of toluene was added. The resulting solution was allowed to stir at ambient temperature and monitored by TLC. The reaction mixture was placed directly onto a silica gel column and purified. The desired product was purified by flash column chromatography and eluted with a suitable solution of hexane and ethyl acetate (typically 4:1). Evaporation of solvent afforded analytically pure product.

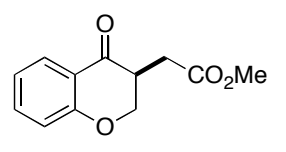

(S)-methyl 2-(4-oxochroman-3-yl)acetate (3): According to the general procedure, $2.5 \mathrm{mg}(0.006 \mathrm{mmol})$ of $\mathbf{4 5} \mathrm{in} 2 \mathrm{~mL}$ of toluene was treated with $0.012 \mathrm{~mL}(0.006 \mathrm{mmol})$ of KHMDS and allowed to stir at ambient temperature for $15 \mathrm{~min} .16 .0 \mathrm{mg}(0.06 \mathrm{mmol})$ of $\mathbf{5 0}$ in $1 \mathrm{~mL}$ toluene was added via syringe. Upon work-up $12.8 \mathrm{mg}(80 \%)$ of desired ester was isolated as a colorless oil.

${ }^{1} \mathrm{H}$ NMR $\left(300 \mathrm{MHz}, \mathrm{CDCl}_{3}\right) \delta 7.88(\mathrm{dd}, 1 \mathrm{H}, J=0.4,6.9 \mathrm{~Hz}), 7.48(\mathrm{dt}, 1 \mathrm{H}, J=1.5,6.9$ $\mathrm{Hz}), 7.02(\mathrm{t}, 1 \mathrm{H}, J=7.6 \mathrm{~Hz}), 6.97(\mathrm{~d}, 1 \mathrm{H}, J=8.4 \mathrm{~Hz}), 4.59(\mathrm{dd}, 1 \mathrm{H}, J=5.3,11.2 \mathrm{~Hz})$, $4.29(\mathrm{t}, 1 \mathrm{H}, J=11.6 \mathrm{~Hz}), 3.73(\mathrm{~s}, 3 \mathrm{H}), 3.34(\mathrm{dddd}, 1 \mathrm{H}, J=5.1,5.1,8.1,12.7 \mathrm{~Hz}), 2.94$ $(\mathrm{dd}, 1 \mathrm{H}, J=4.9,17.0 \mathrm{~Hz}), 2.43(\mathrm{dd}, 1 \mathrm{H}, J=8.1,17.0 \mathrm{~Hz}) ;{ }^{13} \mathrm{C}$ NMR $\left(100 \mathrm{MHz}, \mathrm{CDCl}_{3}\right)$ $\delta 192.8,178.8,172.1,161.9,136.3,127.6,121.6,120.7,118.1,70.4,52.3,42.7,30.3$; Spectral data matched literature description. ${ }^{1}$

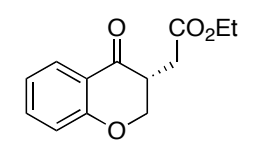

(R)-(4-Oxo-chroman-3-yl)-acetic acid ethyl ester (9): According to the general procedure, $5.0 \mathrm{mg}(0.012 \mathrm{mmol})$ of $4 \mathrm{in} 2 \mathrm{~mL}$ of toluene was treated with $0.024 \mathrm{~mL}(0.012 \mathrm{mmol})$ of KHMDS and allowed to stir at ambient temperature for $15 \mathrm{~min} . \quad 14.0 \mathrm{mg}(0.06 \mathrm{mmol})$ of $8 \mathrm{in} 1 \mathrm{~mL}$ toluene was added via 
syringe. Upon work-up $13.2 \mathrm{mg}$ (94\%) of desired ester was isolated as a colorless oil. Spectral data matched literature description. ${ }^{1}[\alpha]_{\mathrm{D}}{ }^{23}=-10^{\circ}\left(\mathrm{CH}_{2} \mathrm{Cl}_{2}, \mathrm{c}=10 \mathrm{mg} / \mathrm{mL}\right)$; HPLC analysis - Chiralcel AD column, 97:3 hexanes to isopropanol $0.5 \mathrm{ml} / \mathrm{min}$. Major: 30.2 minutes, minor: 40.2 minutes; ${ }^{1} \mathrm{H}$ NMR $\left(300 \mathrm{MHz}, \mathrm{CDCl}_{3}\right) \delta 7.87(\mathrm{dd}, 1 \mathrm{H}, J=1.6$, $7.9 \mathrm{~Hz}), 7.47(\mathrm{dt}, 1 \mathrm{H}, J=1.7,6.9 \mathrm{~Hz}), 7.01(\mathrm{t}, 1 \mathrm{H}, J=7.3 \mathrm{~Hz}), 6.96(\mathrm{~d}, 1 \mathrm{H}, J=8.4 \mathrm{~Hz})$, $4.59(\mathrm{dd}, 1 \mathrm{H}, J=5.3,11.2 \mathrm{~Hz}), 4.29(\mathrm{t}, 1 \mathrm{H}, J=11.8 \mathrm{~Hz}), 4.17(\mathrm{q}, 2 \mathrm{H}, J=7.1 \mathrm{~Hz}), 3.33$ (dddd, $1 \mathrm{H}, J=5.1,5.1,8.1,13.1 \mathrm{~Hz}), 2.92(\mathrm{dd}, 1 \mathrm{H}, J=4.8,17.0 \mathrm{~Hz}), 2.41(\mathrm{dd}, 1 \mathrm{H}, J=$ 8.1, $17.0 \mathrm{~Hz}), 1.27$ (t, 3H, $J=7.1 \mathrm{~Hz}) ;{ }^{13} \mathrm{C} \mathrm{NMR}\left(100 \mathrm{MHz}, \mathrm{CDCl}_{3}\right) \delta$ 192.9, 171.6, $161.9,136.3,127.6,121.7,120.7,118.0,70.4,61.2,42.7,30.6,14.4$.

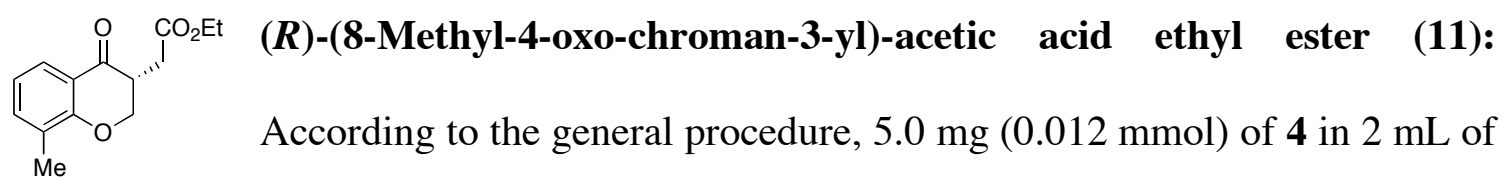
toluene was treated with $0.024 \mathrm{~mL}(0.012 \mathrm{mmol})$ of KHMDS and allowed to stir at ambient temperature for $15 \mathrm{~min} .15 .0 \mathrm{mg}(0.06 \mathrm{mmol})$ of $\mathbf{1 0}$ in $1 \mathrm{~mL}$ toluene was added via syringe. Upon work-up $13.5 \mathrm{mg}(90 \%)$ of desired ester was isolated as a colorless oil. Spectral data matched literature description. ${ }^{1}[\alpha]_{\mathrm{D}}{ }^{23}=-13^{\circ}\left(\mathrm{CH}_{2} \mathrm{Cl}_{2}, \mathrm{c}=11 \mathrm{mg} / \mathrm{mL}\right)$; HPLC analysis - Chiralcel AD column, 97:3 hexanes to isopropanol $0.5 \mathrm{ml} / \mathrm{min}$. Major: 19.4 minutes, minor: 25.1 minutes. ${ }^{1} \mathrm{H}$ NMR $\left(300 \mathrm{MHz}, \mathrm{CDCl}_{3}\right) \delta 7.72(\mathrm{~d}, 1 \mathrm{H}, J=7.8$ $\mathrm{Hz}), 7.32(\mathrm{~d}, 1 \mathrm{H}, J=7.2 \mathrm{~Hz}), 6.90(\mathrm{t}, 1 \mathrm{H}, J=7.6 \mathrm{~Hz}), 4.61(\mathrm{dd}, 1 \mathrm{H}, J=5.3,11.1 \mathrm{~Hz})$, $4.27(\mathrm{t}, 1 \mathrm{H}, J=11.6 \mathrm{~Hz}), 4.18(\mathrm{q}, 2 \mathrm{H}, J=7.1 \mathrm{~Hz}), 3.30(\mathrm{dddd}, 1 \mathrm{H}, J=5.0,5.0,8.4,12.9$ Hz), $2.92(\mathrm{dd}, 1 \mathrm{H}, J=4.8,16.9 \mathrm{~Hz}), 2.39(\mathrm{dd}, 1 \mathrm{H}, J=8.2,16.9 \mathrm{~Hz}), 2.22(\mathrm{~s}, 3 \mathrm{H}), 1.27(\mathrm{t}$, $3 \mathrm{H}, J=7.1 \mathrm{~Hz}) ;{ }^{13} \mathrm{C} \mathrm{NMR}\left(75 \mathrm{MHz}, \mathrm{CDCl}_{3}\right) \delta 193.2,171.6,160.2,137.1,127.3,125.2$, $121.1,120.3,70.3,61.2,42.6,30.6,15.8,14.4$. 


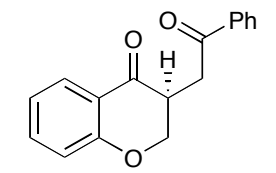

(2S)- 3-(2-Oxo-2-phenyl-ethyl)-chroman-4-one (13): According to the general procedure, $14.0 \mathrm{mg}(0.030 \mathrm{mmol})$ of $\mathbf{4 5}$ and $60.0 \mu \mathrm{L}(0.030$

mmol) of KHMDS and $40.0 \mathrm{mg}(0.150 \mathrm{mmol})$ of $\mathbf{1 2}$ were reacted for 24 hours. Work-up afforded $37.2 \mathrm{mg}(94 \%)$ of the desired product as colorless oil. $\mathrm{R}_{\mathrm{f}}$ (1:1 hexane to ethyl acetate $)=0.7 ;[\alpha]_{\mathrm{D}}^{23}=-36.2\left(\mathrm{CHCl}_{3}, \mathrm{c}=20 \mathrm{mg} / \mathrm{mL}\right) ; \mathrm{HPLC}$ analysis - Chiralcel OD-H column 90:10 hexanes to isopropanol $1.0 \mathrm{~mL} / \mathrm{min}$. Major enantiomer: 10.9 minutes, Minor enantiomer: 13.9 minutes; ${ }^{1} \mathrm{H}$ NMR $\left(400 \mathrm{MHz}, \mathrm{CDCl}_{3}\right) \delta$ 7.99-7.97 $(2 \mathrm{H}, \mathrm{m}), 7.88$ $(1 \mathrm{H}, \mathrm{m}), 7.56(1 \mathrm{H}, \mathrm{m}), 7.49-7.44(3 \mathrm{H}, \mathrm{m}), 7.01(1 \mathrm{H}, \mathrm{m}), 6.97(1 \mathrm{H}, \mathrm{m}), 4.62(1 \mathrm{H}, \mathrm{dd}, J=$ 5.3, $11.1 \mathrm{~Hz}), 4.29(1 \mathrm{H}, \mathrm{dd}, J=11.3,11.5 \mathrm{~Hz}), 3.69(1 \mathrm{H}, \mathrm{dd}, J=3.8,18.1 \mathrm{~Hz}), 3.58(1 \mathrm{H}$, dddd, $J=3.8,5.3,8.5,11.9 \mathrm{~Hz}), 3.00(1 \mathrm{H}, \mathrm{d}, J=8.3,17.9 \mathrm{~Hz}) ;{ }^{13} \mathrm{C} \mathrm{NMR}(100 \mathrm{MHz}$, $\left.\mathrm{CDCl}_{3}\right) \delta 197.2,193.6,162.0,136.6,136.2,133.6,128.9,128.3,127.6,121.6,120.8$, 118.0, 70.6, 42.0, 34.5; IR (NaCl, $\left.\mathrm{CH}_{2} \mathrm{Cl}_{2}\right) 1680,1607,1483 \mathrm{~cm}^{-1}$; HRMS (FAB+) calcd for $\mathrm{C}_{17} \mathrm{H}_{15} \mathrm{O}_{3}(\mathrm{M}+\mathrm{H})^{+}:$267.1021, Found 267.1028.

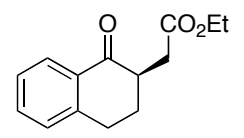

$(R)$-(1-Oxo-1,2,3,4-tetrahydro-naphthalen-2-yl)-acetic acid ethyl ester

(15): According to the general procedure, $5.0 \mathrm{mg}(1.2 \mathrm{mmol})$ of 6 in $2 \mathrm{~mL}$ of toluene was treated with $0.024 \mathrm{~mL}(0.012 \mathrm{mmol})$ of KHMDS and allowed to stir at ambient temperature for $15 \mathrm{~min} .14 .0 \mathrm{mg}(0.06 \mathrm{mmol})$ of $\mathbf{1 4} \mathrm{in} 1 \mathrm{~mL}$ toluene was added via syringe. Upon work-up $12.5 \mathrm{mg}(90 \%)$ of desired ester was isolated as a colorless oil; $\mathrm{R}_{\mathrm{f}}(3: 1$ hexanes to ethyl acetate $)=0.47 ;[\alpha]_{\mathrm{D}}^{23}=+15.5^{\circ}\left(\mathrm{CH}_{2} \mathrm{Cl}_{2}, \mathrm{c}=9 \mathrm{mg} / \mathrm{mL}\right) ;$ HPLC analysis - Chiralcel AD column, 97:3 hexanes to isopropanol $0.5 \mathrm{ml} / \mathrm{min}$. Minor: 21.6 minutes, major: 23.7 minutes; ${ }^{1} \mathrm{H}$ NMR $\left(300 \mathrm{MHz}, \mathrm{CDCl}_{3}\right) \delta 8.02(\mathrm{dd}, 1 \mathrm{H}, J=8.1,1.2$ 
$\mathrm{Hz}), 7.46(\mathrm{ddd}, 1 \mathrm{H}, J=7.5,7.5,1.5 \mathrm{~Hz}), 7.21-7.33(\mathrm{~m}, 2 \mathrm{H}), 4.18(\mathrm{q}, 2 \mathrm{H}, J=6.5 \mathrm{~Hz})$, $2.93-3.19(\mathrm{~m}, 4 \mathrm{H}), 2.42(\mathrm{dd}, 1 \mathrm{H}, J=18.3,8.8 \mathrm{~Hz}), 2.25(\mathrm{dddd}, 1 \mathrm{H}, J=3.0,4.4,4.4$, $12.6 \mathrm{~Hz}), 1.97(\mathrm{~m}, 1 \mathrm{H}), 1.28(\mathrm{t}, 3 \mathrm{H}, J=7.3 \mathrm{~Hz}) ;{ }^{13} \mathrm{C} \mathrm{NMR}\left(75 \mathrm{MHz}, \mathrm{CDCl}_{3}\right) \delta$ 198.4, 172.7, 133.6, 129.0, 129.0, 128.9, 127.8, 126.8, 60.9, 45.2, 45.1, 35.5, 29.6, 14.6; IR (NaCl) 2983, 1730, 1678, 1599, 1161, $762 \mathrm{~cm}^{-1}$; HRMS (FAB+) calcd for $\mathrm{C}_{14} \mathrm{H}_{16} \mathrm{O}_{3}$, 233.1178. Found 233.1167.

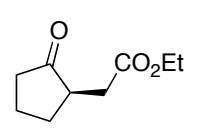

(R)-(2-Oxo-cyclopentyl)-acetic acid ethyl ester (17): ${ }^{5}$ According to the general procedure, $5 \mathrm{mg}(0.013 \mathrm{mmol})$ of 6 in $2 \mathrm{~mL}$ of toluene was treated with $0.026 \mathrm{~mL}(0.013 \mathrm{mmol})$ of KHMDS and allowed to stir at ambient temperature for $15 \mathrm{~min} .10 .0 \mathrm{mg}(0.065 \mathrm{mmol})$ of $\mathbf{1 6} \mathrm{in} 1 \mathrm{~mL}$ toluene was added via syringe. Upon work-up $4.0 \mathrm{mg}(81 \%)$ of desired ester was isolated as a colorless oil: $[\alpha]_{\mathrm{D}}^{23}=-65^{\circ}$ $\left(\mathrm{CH}_{2} \mathrm{Cl}_{2}, \mathrm{c}=7 \mathrm{mg} / \mathrm{mL}\right)$; Gas chromatography analysis - B-DM column with constant 110 ${ }^{\circ} \mathrm{C}$ oven temperature. Major: 19.6 minutes, minor: $20.5 \mathrm{~min} .{ }^{1} \mathrm{H}$ NMR (400 MHz, $\left.\mathrm{CDCl}_{3}\right) \delta 4.11(\mathrm{q}, 2 \mathrm{H}, J=7.1 \mathrm{~Hz}), 2.72-2.68(\mathrm{~m}, 1 \mathrm{H}), 2.46-2.24(\mathrm{~m}, 4 \mathrm{H}), 2.20-2.10(\mathrm{~m}$, 1H), 2.07- $1.99(\mathrm{~m}, 1 \mathrm{H}), 1.84-1.72(\mathrm{~m}, 1 \mathrm{H}), 1.64-1.54(\mathrm{~m}, 1 \mathrm{H}), 1.22(\mathrm{t}, 3 \mathrm{H}, J=7.1 \mathrm{~Hz})$; ${ }^{13} \mathrm{C}$ NMR $\left(100 \mathrm{MHz}, \mathrm{CDCl}_{3}\right) \delta$ 219.5, 172.3, 60.8, 45.9, 37.7, 34.2, 29.5, 20.8, 14.4.

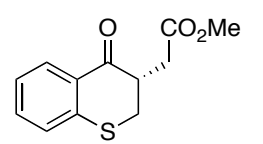

(S)-(4-Oxo-thiochroman-3-yl)-acetic acid methyl ester (19): According to the general procedure, $5.0 \mathrm{mg}(0.012 \mathrm{mmol})$ of 4 in $2 \mathrm{~mL}$ of toluene was treated with $0.024 \mathrm{~mL}(0.012 \mathrm{mmol})$ of KHMDS $(0.5 \mathrm{M}$ in toluene) and allowed to stir at ambient temperature for $15 \mathrm{~min}$, to which was added $14.0 \mathrm{mg}(0.06$ mmol) of 18 in $1 \mathrm{~mL}$ toluene via syringe. Upon work-up $9.0 \mathrm{mg}(63 \%)$ of desired ester was isolated as a colorless oil: $R_{\mathrm{f}}(3: 1$ hexanes to ethyl acetate $)=0.42 ;[\alpha]_{\mathrm{D}}{ }^{23}=-107.5^{\circ}$ 
$\left(\mathrm{CH}_{2} \mathrm{Cl}_{2}, \mathrm{c}=9 \mathrm{mg} / \mathrm{mL}\right) ; \quad$ HPLC analysis - Chiralcel AD column, 97:3 hexanes to isopropanol $0.5 \mathrm{ml} / \mathrm{min}$. Major: 28.9 minutes, minor: 33.5 minutes; ${ }^{1} \mathrm{H}$ NMR $(300 \mathrm{MHz}$, $\left.\mathrm{CDCl}_{3}\right) \delta 8.08(\mathrm{dd}, 1 \mathrm{H}, J=8.0,1.4 \mathrm{~Hz}), 7.83(\mathrm{ddd}, 1 \mathrm{H}, J=8.1,7.1,1.8 \mathrm{~Hz}), 7.14-7.27$ $(\mathrm{m}, 2 \mathrm{H}), 3.73(\mathrm{~s}, 3 \mathrm{H}), 3.37(\mathrm{ddd}, 1 \mathrm{H}, J=9.0,4.8,2.0 \mathrm{~Hz}), 3.34(\mathrm{dd}, 1 \mathrm{H}, J=12.8,12.8$ $\mathrm{Hz}), 3.11(\mathrm{~m}, 1 \mathrm{H}), 2.99(\mathrm{dd}, 1 \mathrm{H}, J=16.8,5.2 \mathrm{~Hz}), 2.59(\mathrm{dd}, 1 \mathrm{H}, J=16.6,6.8 \mathrm{~Hz}) ;{ }^{13} \mathrm{C}$ NMR $\left(75 \mathrm{MHz}, \mathrm{CDCl}_{3}\right) \delta 194.3,172.0,141.6,133.2,130.4,129.6,127.3,124.9,52.0$, 44.6, 34.1, 31.0; IR (NaCl) 2916, 1732, 1674, 1587, 1435, $1167 \mathrm{~cm}^{-1}$; HRMS (FAB+) calcd for $\mathrm{C}_{12} \mathrm{H}_{12} \mathrm{O}_{3} \mathrm{~S}, 237.0585$. Found 237.0585.

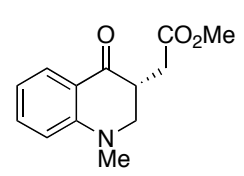

\section{(R)-(1-Methyl-4-oxo-1,2,3,4-tetrahydro-quinolin-3-yl)-acetic}

acid ethyl ester (21): According to the general procedure, $5.0 \mathrm{mg}(0.012$ $\mathrm{mmol})$ of 4 in $2 \mathrm{~mL}$ of toluene was treated with $0.024 \mathrm{~mL}(0.012 \mathrm{mmol})$ of KHMDS and allowed to stir at ambient temperature for $15 \mathrm{~min} .14 .0 \mathrm{mg}(0.06 \mathrm{mmol})$ of 20 in $1 \mathrm{~mL}$ toluene was added via syringe. Upon work-up $9.0 \mathrm{mg}(64 \%)$ of desired ester was isolated as a light yellow oil; $\operatorname{Rf}(3: 1$ hexanes to ethyl acetate $)=0.25 ;[\alpha]_{\mathrm{D}}^{23}=-46.6^{\circ}\left(\mathrm{CH}_{2} \mathrm{Cl}_{2}, \mathrm{c}=\right.$ $9 \mathrm{mg} / \mathrm{mL}$ ); HPLC analysis - Chiralcel OD-H column, 90:10 hexanes to isopropanol 0.5 ml/min. Major: 29.6 minutes, minor: 38.7 minutes; ${ }^{1} \mathrm{H}$ NMR $\left(300 \mathrm{MHz}, \mathrm{CDCl}_{3}\right) \delta 7.90$ (dd, 1H, $J=7.9,1.6 \mathrm{~Hz}), 7.41(\mathrm{ddd}, 1 \mathrm{H}, J=8.6,7.1,1.7 \mathrm{~Hz}), 6.69-6.79(\mathrm{~m}, 2 \mathrm{H}), 3.73(\mathrm{~s}$, $3 \mathrm{H}), 3.50(\mathrm{dd}, 1 \mathrm{H}, J=11.4,5.4 \mathrm{~Hz}), 3.33(\mathrm{dd}, 1 \mathrm{H}, J=12.2,12.2 \mathrm{~Hz}), 3.22(\mathrm{~m}, 1 \mathrm{H}), 3.03$ $(\mathrm{dd}, 1 \mathrm{H}, J=16.4,4.86 \mathrm{~Hz}), 3.00(\mathrm{~s}, 3 \mathrm{H}), 2.36(\mathrm{dd}, 1 \mathrm{H}, J=14.4,8.5 \mathrm{~Hz}) ;{ }^{13} \mathrm{C} \mathrm{NMR}(75$ $\left.\mathrm{MHz}, \mathrm{CDCl}_{3}\right) \delta 194.1,172.8,135.5,129.7,129.0,128.6,117.4,113.3,56.0,52.1,43.3$, 39.5, 31.8; IR $\left(\mathrm{NaCl}, \mathrm{CH}_{2} \mathrm{Cl}_{2}\right)$ 2953, 1738, 1674, 1605, 1498, 1342, $754 \mathrm{~cm}^{-1}$; HRMS (FAB+) calcd for $\mathrm{C}_{13} \mathrm{H}_{15} \mathrm{NO}_{3}, 234.1130$. Found 234.1123. 


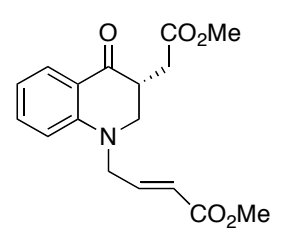

\section{(R)-4-(3-Methoxycarbonylmethyl-4-oxo-3,4-dihydro-2H-quinolin-1-} yl)-but-2-enoic acid methyl ester (23): According to the general

procedure, $5.0 \mathrm{mg}(0.012 \mathrm{mmol})$ of $4 \mathrm{in} 2 \mathrm{~mL}$ of toluene was treated with $0.024 \mathrm{~mL}(0.012 \mathrm{mmol})$ of KHMDS and allowed to stir at ambient temperature for $15 \mathrm{~min} .19 .0 \mathrm{mg}(0.06 \mathrm{mmol})$ of $\mathbf{2 2} \mathrm{in} 1 \mathrm{~mL}$ toluene was added via syringe. Upon workup $13.7 \mathrm{mg}(72 \%)$ of desired ester was isolated as a light yellow oil; $\mathrm{R}_{\mathrm{f}}(3: 1$ hexanes to ethyl acetate $)=0.12 ;[\alpha]_{\mathrm{D}}{ }^{23}=-20.8^{\circ}\left(\mathrm{CH}_{2} \mathrm{Cl}_{2}, \mathrm{c}=10 \mathrm{mg} / \mathrm{mL}\right) ; \mathrm{HPLC}$ analysis - Chiralcel AD column, 90:10 hexanes to isopropanol $0.5 \mathrm{ml} / \mathrm{min}$. Minor: 42.3 minutes, major: 45.7 minutes; ${ }^{1} \mathrm{H}$ NMR $\left(300 \mathrm{MHz}, \mathrm{CDCl}_{3}\right) \delta 7.91(\mathrm{dd}, 1 \mathrm{H}, J=8.2,1.6 \mathrm{~Hz}), 7.37$ (ddd, $1 \mathrm{H}, J=$ 8.5, 6.8, 1.5 Hz), $6.92(\mathrm{ddd}, 1 \mathrm{H}, J=15.8,15.8,4.9 \mathrm{~Hz}), 6.77(\mathrm{ddd}, 1 \mathrm{H}, J=8.1,8.1,0.7$ $\mathrm{Hz}), 6.60(\mathrm{~d}, 1 \mathrm{H}, J=8.7 \mathrm{~Hz}), 5.98(\mathrm{ddd}, 1 \mathrm{H}, J=15.7,15.7,1.86 \mathrm{~Hz}), 4.16(\mathrm{ddd}, 1 \mathrm{H}, J=$ $18.2,4.2,1.7 \mathrm{~Hz}), 4.14(\mathrm{ddd}, 1 \mathrm{H}, J=18.1,4.1,1.8 \mathrm{~Hz}), 3.72(\mathrm{~s}, 6 \mathrm{H}), 3.52(\mathrm{dd}, 1 \mathrm{H}, J=$ $11.8,6.5), 3.48(\mathrm{dd}, 1 \mathrm{H}, J=18.2,11.8 \mathrm{~Hz}), 3.23(\mathrm{~m} \mathrm{1H}), 3.02(\mathrm{dd}, 1 \mathrm{H}, J=16.8,4.8 \mathrm{~Hz})$, $2.36(\mathrm{dd}, 1 \mathrm{H}, J=16.9,8.1 \mathrm{~Hz}) ;{ }^{13} \mathrm{C} \mathrm{NMR}\left(100 \mathrm{MHz}, \mathrm{CDCl}_{3}\right) \delta 193.4,172.4,166.2$, $142.3,135.6,128.7,127.9,122.7,119.2,117.7,113.1,53.9,52.4,51.9,51.7,42.8,31.5$; IR (NaCl) 2953, 1728, 1674, 1605, 1497, 1173, $754 \mathrm{~cm}^{-1}$; HRMS (FAB+) calcd for $\mathrm{C}_{17} \mathrm{H}_{19} \mathrm{NO}_{5}, 318.1341$. Found 318.1340.

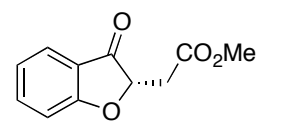

Methyl 2-(3-oxo-2,3-dihydrobenzofuran-2-yl)acetate (27). According to the general procedure, $2.5 \mathrm{mg}(0.006 \mathrm{mmol})$ of 4 in $2 \mathrm{~mL}$ of toluene was treated with $0.012 \mathrm{~mL}(0.006 \mathrm{mmol})$ of KHMDS and allowed to stir at ambient temperature for $15 \mathrm{~min} .16 .0 \mathrm{mg}(0.06 \mathrm{mmol})$ of $\mathbf{2 6}$ in $1 \mathrm{~mL}$ toluene was added via syringe. Upon work-up $14.4 \mathrm{mg}(90 \%)$ of desired ester was isolated as a colorless oil. ${ }^{1} \mathrm{H}$ 
NMR (300 MHz, $\left.\mathrm{CDCl}_{3}\right) \delta$ 7.70-7.59 (m, 2H), 7.15-7.08 (m, 2H), 4.90 (dd, 1H, $J=3.6$, $8.0 \mathrm{~Hz}), 3.72(\mathrm{~s}, 3 \mathrm{H}), 3.09(\mathrm{dd}, 1 \mathrm{H}, J=3.6,17.1 \mathrm{~Hz}), 2.80(\mathrm{dd}, 1 \mathrm{H}, J=8.1,17.0 \mathrm{~Hz}){ }^{13} \mathrm{C}$ NMR (75 MHz, $\left.\mathrm{CDCl}_{3}\right) \delta 200.4,172.6,170.1,138.3,124.5,122.4,120.9,113.7,81.2$, $52.4,36.0$.

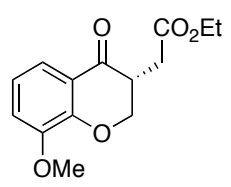

(R)-(8-Methoxy-4-oxo-chroman-3-yl)-acetic acid ethyl ester (29): According to the general procedure, $2.5 \mathrm{mg}(0.006 \mathrm{mmol})$ of $\mathbf{4}$ in $2 \mathrm{~mL}$ of toluene was treated with $0.012 \mathrm{~mL}(0.006 \mathrm{mmol})$ of KHMDS and allowed to stir at ambient temperature for $15 \mathrm{~min} .16 .0 \mathrm{mg}(0.06 \mathrm{mmol})$ of $\mathbf{2 8} \mathrm{in} 1 \mathrm{~mL}$ toluene was added via syringe. Upon work-up $15.0 \mathrm{mg}$ (95\%) of desired ester was isolated as a colorless oil. Spectral data matched literature description. ${ }^{1}[\alpha]_{\mathrm{D}}{ }^{23}=-15^{\circ}\left(\mathrm{CH}_{2} \mathrm{Cl}_{2}, \mathrm{c}=12 \mathrm{mg} / \mathrm{mL}\right)$; HPLC analysis - Chiralcel AD column, 97:3 hexanes to isopropanol $1.0 \mathrm{ml} / \mathrm{min}$. Major: 27.8 minutes, minor: 42.2 minutes. ${ }^{1} \mathrm{H}$ NMR $\left(300 \mathrm{MHz}, \mathrm{CDCl}_{3}\right) \delta 7.47(\mathrm{dd}, 1 \mathrm{H}, J=1.5$, $7.9 \mathrm{~Hz}), 7.04(\mathrm{dd}, 1 \mathrm{H}, J=1.5,7.9 \mathrm{~Hz}), 6.95(\mathrm{t}, 1 \mathrm{H}, J=7.9 \mathrm{~Hz}), 4.69$ (dd, $1 \mathrm{H}, J=5.2$, $11.2 \mathrm{~Hz}$ ), 4.36 (t, 1H, $J=11.6 \mathrm{~Hz}$ ), 4.17 (q, $2 \mathrm{H}, J=7.1 \mathrm{~Hz}), 3.89$ (s, 3H), 3.37 (dddd, $1 \mathrm{H}$, $J=5.1,5.1,8.1,13.1 \mathrm{~Hz}), 2.89(\mathrm{dd}, 1 \mathrm{H}, J=4.9,17.0 \mathrm{~Hz}), 4.43(\mathrm{dd}, 1 \mathrm{H}, J=8.1,17.0$ $\mathrm{Hz}), 1.26(\mathrm{t}, 3 \mathrm{H}, J=7.1 \mathrm{~Hz}) ;{ }^{13} \mathrm{C} \mathrm{NMR}\left(100 \mathrm{MHz}, \mathrm{CDCl}_{3}\right) \delta 192.8,171.4,170.5,151.8$, $148.9,121.2,118.7,116.8,70.9,61.2,56.4,42.6,30.5,14.4$.

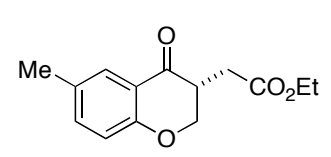

(6-Methyl-4-oxo-chroman-3-yl)-acetic acid ethyl ester (31):

According to the general procedure, $2.5 \mathrm{mg}(0.006 \mathrm{mmol})$ of 4 in 2 $\mathrm{mL}$ of toluene was treated with $0.012 \mathrm{~mL}(0.012 \mathrm{mmol})$ of KHMDS and allowed to stir at ambient temperature for $15 \mathrm{~min} .15 .0 \mathrm{mg}(0.06 \mathrm{mmol})$ of $\mathbf{3 0}$ in $1 \mathrm{~mL}$ toluene was added via syringe. Upon work-up $12 \mathrm{mg}(80 \%)$ of desired ester was isolated as a colorless oil. 
Spectral data matched literature description. ${ }^{1}[\alpha]_{\mathrm{D}}{ }^{23}=-11^{\circ}\left(\mathrm{CH}_{2} \mathrm{Cl}_{2}, \mathrm{c}=10 \mathrm{mg} / \mathrm{mL}\right)$; HPLC analysis - Chiralcel AD column, 97:3 hexanes to isopropanol $0.5 \mathrm{ml} / \mathrm{min}$. Major: 25.7 minutes, minor: 39.2 minutes. ${ }^{1} \mathrm{H}$ NMR $\left(300 \mathrm{MHz}, \mathrm{CDCl}_{3}\right) \delta 7.67(\mathrm{~d}, 1 \mathrm{H}, J=1.8$ $\mathrm{Hz}), 7.28(\mathrm{dd}, 1 \mathrm{H}, J=2.3,8.4 \mathrm{~Hz}), 6.87(\mathrm{~d}, 1 \mathrm{H}, J=8.4 \mathrm{~Hz}), 4.56(\mathrm{dd}, 1 \mathrm{H}, J=5.3,11.1$ $\mathrm{Hz}), 4.26(\mathrm{t}, 1 \mathrm{H}, J=11.6 \mathrm{~Hz}), 4.17$ (q, 2H, $J=7.1 \mathrm{~Hz}), 3.30$ (dddd, 1H, $J=5.0,5.0,8.2$, $10.0 \mathrm{~Hz}), 2.91(\mathrm{dd}, 1 \mathrm{H}, J=4.8,16.9 \mathrm{~Hz}), 2.40(\mathrm{dd}, 1 \mathrm{H}, J=8.2,16.9 \mathrm{~Hz}), 2.29(\mathrm{~s}, 3 \mathrm{H})$, $1.27(\mathrm{t}, 3 \mathrm{H}, J=7.1 \mathrm{~Hz}) ;{ }^{13} \mathrm{C} \mathrm{NMR}\left(100 \mathrm{MHz}, \mathrm{CDCl}_{3}\right) \delta 193.1,171.7,160.0,137.3,131.2$, $127.3,120.3,117.8,70.4,61.2,42.7,30.6,20.6,14.5$.

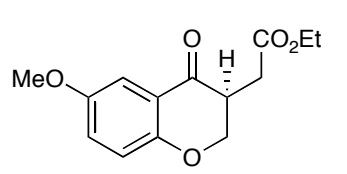

(2S)-(6-Methoxy-4-oxo-chroman-3-yl)-acetic acid ethyl ester (33).

According to the general procedure, $10.0 \mathrm{mg}(0.028 \mathrm{mmol})$ of $\mathbf{6}, 57.0$ $\mu \mathrm{L}(0.028 \mathrm{mmol})$ of KHMDS and $37.0 \mathrm{mg}(0.14 \mathrm{mmol})$ of $\mathbf{3 2}$ were reacted for 24 hours. Work-up afforded $35.0 \mathrm{mg}(94 \%)$ of the desired product as colorless oil. $\mathrm{R}_{\mathrm{f}}(1: 1$ hexane to ethyl acetate $)=0.6 ;[\alpha]_{\mathrm{D}}^{23}=+23.8\left(\mathrm{CHCl}_{3}, \mathrm{c}=10 \mathrm{mg} / \mathrm{mL}\right) ; \mathrm{HPLC}$ analysis - Chiralcel AD column 95:5 hexanes to isopropanol $1.0 \mathrm{~mL} / \mathrm{min}$. Minor enantiomer: 14.0 minutes. Major enantiomer: 19.0 minutes; ${ }^{1} \mathrm{H}$ NMR $\left(400 \mathrm{MHz}, \mathrm{CDCl}_{3}\right) \delta 7.29(1 \mathrm{H}, \mathrm{d}, J=3.3 \mathrm{~Hz})$, $7.07(1 \mathrm{H}, \mathrm{d}, J=3.2 \mathrm{~Hz}), 6.89(1 \mathrm{H}, \mathrm{d}, J=9.0 \mathrm{~Hz}), 4.54(1 \mathrm{H}, \mathrm{dd}, J=5.2,11.2 \mathrm{~Hz}), 4.25(1 \mathrm{H}$, dd, $J=11.2,11.7 \mathrm{~Hz}), 4.17(2 \mathrm{H}, \mathrm{q}, J=7.2 \mathrm{~Hz}), 3.78(3 \mathrm{H}, \mathrm{s}) 3.29(1 \mathrm{H}$, dddd, $J=5.1,8.1$, 11.0, 13.0 Hz), $2.90(1 \mathrm{H}, \mathrm{dd}, J=5.0,17.0 \mathrm{~Hz}), 2.40(1 \mathrm{H}, \mathrm{dd}, J=7.8,17.0 \mathrm{~Hz}), 1.27(3 \mathrm{H}, \mathrm{t}$, $J=7.2 \mathrm{~Hz}) ;{ }^{13} \mathrm{C}$ NMR $\left(100 \mathrm{MHz}, \mathrm{CDCl}_{3}, \mathrm{c}=10 \mathrm{mg} / \mathrm{mL}\right) \delta 192.5,171.2,153.9,125.1$, 119.0, 107.5, 70.3, 61.0, 55.8, 42.5, 30.5, 14.3; IR ( $\left.\mathrm{NaCl}, \mathrm{CH}_{2} \mathrm{Cl}_{2}\right)$ 1723, 1687, 1490, 1432, $1272 \mathrm{~cm}^{-1}$; HRMS (FAB+) calcd for $\mathrm{C}_{14} \mathrm{H}_{17} \mathrm{O}_{5}(\mathrm{M}+\mathrm{H})^{+}:$265.1076. Found 265.1068. 


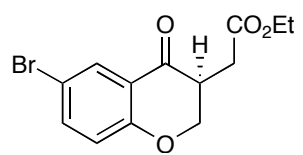

(2S)- (6-Bromo-4-oxo-chroman-3-yl)-acetic acid ethyl ester (35)

According to the general procedure, $11.6 \mathrm{mg}(0.032 \mathrm{mmol})$ of $\mathbf{6}$ and $64.0 \mu \mathrm{L}(0.032 \mathrm{mmol})$ of KHMDS and $50.0 \mathrm{mg}(0.160 \mathrm{mmol})$ of $\mathbf{3 4}$ were reacted for 20 minutes. Work-up afforded $47.5 \mathrm{mg}(95 \%)$ of the desired product as colorless oil. $\mathrm{R}_{\mathrm{f}}(1: 1$ hexane to ethyl acetate $)=0.6 ;[\alpha]_{\mathrm{D}}^{23}=+10.2\left(\mathrm{CHCl}_{3}, \mathrm{c}=10 \mathrm{mg} / \mathrm{mL}\right) ;$ HPLC analysis Chiralcel OD-H column 97:3 hexanes to isopropanol $0.5 \mathrm{~mL} / \mathrm{min}$. Major enantiomer: 24.6 minutes, Minor enantiomer: 26.3 minutes; ${ }^{1} \mathrm{H}$ NMR $\left(400 \mathrm{MHz}, \mathrm{CDCl}_{3}\right) \delta 7.95(1 \mathrm{H}$, d, $J=2.5 \mathrm{~Hz}), 7.51(1 \mathrm{H}, \mathrm{dd}, J=2.5,8.7 \mathrm{~Hz}), 6.85(1 \mathrm{H}, \mathrm{d}, J=8.9 \mathrm{~Hz}), 4.58(1 \mathrm{H}, \mathrm{dd}, J=5.3$, $11.3 \mathrm{~Hz}), 4.26(1 \mathrm{H}, \mathrm{dd}, J=11.1,11.5 \mathrm{~Hz}), 4.15(2 \mathrm{H}, \mathrm{q}, J=7.2 \mathrm{~Hz}), 3.28(1 \mathrm{H}, \mathrm{dddd}, J=5.1$, 8.7, 9.8, 13.0 Hz), $2.87(1 \mathrm{H}, \mathrm{dd}, J=4.6,17.0 \mathrm{~Hz}), 2.39(1 \mathrm{H}, \mathrm{dd}, J=7.8,17.0 \mathrm{~Hz}), 1.25$ $(3 \mathrm{H}, \mathrm{t}, J=7.2 \mathrm{~Hz}) ;{ }^{13} \mathrm{C}$ NMR $\left(100 \mathrm{MHz}, \mathrm{CDCl}_{3}\right) \delta 191.3,171.0,160.5,138.5,129.7$, 121.7, 119.9, 114.1, 70.2, 61.0, 42.2, 30.1, 14.1; IR $\left(\mathrm{NaCl}, \mathrm{CH}_{2} \mathrm{Cl}_{2}\right)$ 1723, 1687, 1600, $1469 \mathrm{~cm}^{-1}$; HRMS (FAB+) calcd for $\mathrm{C}_{13} \mathrm{H}_{14} \mathrm{BrO}_{4}(\mathrm{M}+\mathrm{H})^{+}: 313.0075$, Found 313.0059.

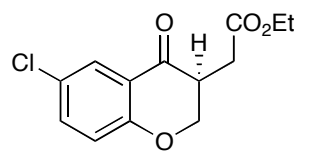

(2S)- (6-Chloro-4-oxo-chroman-3-yl)-acetic acid ethyl ester (37) According to the general procedure, $13.5 \mathrm{mg}(0.037 \mathrm{mmol})$ of $\mathbf{6}$ and $76.0 \mu \mathrm{L}(0.037 \mathrm{mmol})$ of KHMDS and $50.0 \mathrm{mg}(0.186 \mathrm{mmol})$ of $\mathbf{3 6}$ were reacted for 20 minutes. Work-up afforded $47.0 \mathrm{mg}(94 \%)$ of the desired product as colorless oil. $\mathbf{R}_{\mathrm{f}}(1: 1$ hexane to ethyl acetate $)=0.6 ;[\alpha]_{\mathrm{D}}^{23}=+1.4\left(\mathrm{CHCl}_{3}, \mathrm{c}=5 \mathrm{mg} / \mathrm{mL}\right)$; HPLC analysisChiralcel AD column 95:5 hexanes to isopropanol $1 \mathrm{~mL} / \mathrm{min}$. Major enantiomer: 10.9 minutes, Minor enantiomer: 15.0 minutes; ${ }^{1} \mathrm{H}$ NMR $\left(400 \mathrm{MHz}, \mathrm{CDCl}_{3}\right) \delta 7.80(1 \mathrm{H}, \mathrm{d}, J=$ $2.5 \mathrm{~Hz}), 7.38(1 \mathrm{H}, \mathrm{dd}, J=2.5,8.9 \mathrm{~Hz}), 6.90(1 \mathrm{H}, \mathrm{d}, J=8.9 \mathrm{~Hz}), 4.57(1 \mathrm{H}, \mathrm{dd}, J=5.3,11.1$ Hz), $4.26(1 \mathrm{H}, \mathrm{dd}, J=11.5,11.9 \mathrm{~Hz}), 4.15(2 \mathrm{H}, \mathrm{q}, J=7.2 \mathrm{~Hz}), 3.28(1 \mathrm{H}, \mathrm{dddd}, J=5.1,8.1$, 
$10.2,13.0 \mathrm{~Hz}), 2.88(1 \mathrm{H}, \mathrm{dd}, J=4.6,17.0 \mathrm{~Hz}), 2.39(1 \mathrm{H}, \mathrm{dd}, J=8.1,17.0 \mathrm{~Hz}), 1.25(3 \mathrm{H}, \mathrm{t}$, $J=7.2 \mathrm{~Hz}) ;{ }^{13} \mathrm{C} \mathrm{NMR}\left(100 \mathrm{MHz}, \mathrm{CDCl}_{3}\right) \delta 191.5,171.1,160.1,138.8,127.0,126.6$, 121.2, 119.6, 70.3, 61.0, 42.3, 30.2, 14.1; IR $\left(\mathrm{NaCl}, \mathrm{CH}_{2} \mathrm{Cl}_{2}\right)$ 1730, 1701, 1469, $1418 \mathrm{~cm}^{-}$ ${ }^{1}$; HRMS (FAB+) calcd for $\mathrm{C}_{13} \mathrm{H}_{14} \mathrm{ClO}_{4}(\mathrm{M}+\mathrm{H})^{+}$: 269.0581, Found 269.0580.

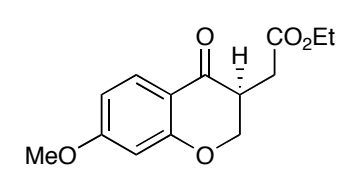

(2S)- (7-Methoxy-4-oxo-chroman-3-yl)-acetic acid ethyl ester

(39) According to the general procedure, $10.0 \mathrm{mg}(0.028 \mathrm{mmol})$ of 6 and $57.0 \mu \mathrm{L}(0.028 \mathrm{mmol})$ of KHMDS and $38.0 \mathrm{mg}(0.143 \mathrm{mmol})$ of $\mathbf{3 8}$ were reacted for 60 minutes. Work-up afforded $17.1 \mathrm{mg}(45 \%)$ of the desired product as colorless oil. $\mathrm{R}_{\mathrm{f}}$ $(1: 1$ hexane to ethyl acetate $)=0.7 ;[\alpha]_{\mathrm{D}}{ }^{23}=+8.0\left(\mathrm{CHCl}_{3}, \mathrm{c}=10 \mathrm{mg} / \mathrm{mL}\right) ; \mathrm{HPLC}$ analysis - Chiralcel AD-H column 95:5 hexanes to isopropanol 1.0 mL/min. Major enantiomers: 36.24 minutes: Minor Enantiomer: 27.61 minutes; ${ }^{1} \mathrm{H}$ NMR $\left(400 \mathrm{MHz}, \mathrm{CDCl}_{3}\right) \delta$ 7.89(1H, d, $J=8.7 \mathrm{~Hz}), 6.55(1 \mathrm{H}, \mathrm{dd}, J=2.3,8.7 \mathrm{~Hz}), 6.38(1 \mathrm{H}, \mathrm{d}, J=2.3 \mathrm{~Hz}), 4.55(1 \mathrm{H}$, dd, $J=5.3,11.0 \mathrm{~Hz}), 4.24(1 \mathrm{H}, \mathrm{dd}, J=11.2,11.2 \mathrm{~Hz}), 4.15(2 \mathrm{H}, \mathrm{q}, J=7.1 \mathrm{~Hz}), 3.80(3 \mathrm{H}$, s), $3.24(1 \mathrm{H}$, dddd, $J=4.9,8.3,9.8,11.7 \mathrm{~Hz}), 2.90(1 \mathrm{H}, \mathrm{dd}, J=4.7,17.0 \mathrm{~Hz}), 2.35(1 \mathrm{H}$, dd, $J=8.5,17.0 \mathrm{~Hz}), 1.25(3 \mathrm{H}, \mathrm{t}, J=7.2 \mathrm{~Hz}) ;{ }^{13} \mathrm{C} \mathrm{NMR}\left(100 \mathrm{MHz}, \mathrm{CDCl}_{3}\right) \delta 191.2,171.5$, 165.9, 163.6, 129.0, 114.3, 110.0, 100.6, 70.5, 60.9, 55.6, 42.0, 30.4, 14.1; IR (NaCl, $\left.\mathrm{CH}_{2} \mathrm{Cl}_{2}\right)$ 1730, 1680, 1600, $1265 \mathrm{~cm}^{-1}$; HRMS (FAB+) calcd for $\mathrm{C}_{14} \mathrm{H}_{17} \mathrm{O}_{5}(\mathrm{M}+\mathrm{H})^{+}$: 265.1076, Found 265.1062.

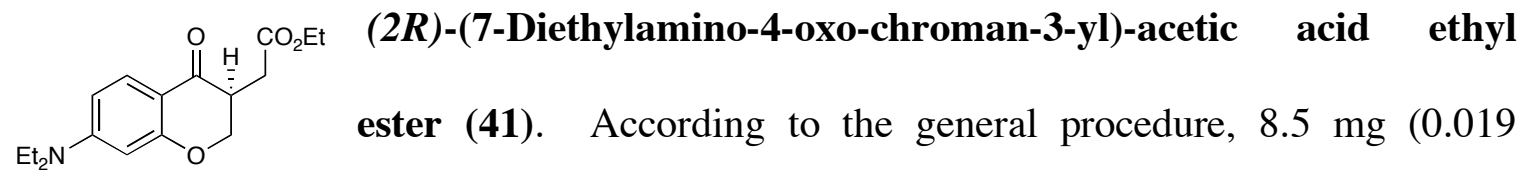
$\mathrm{mmol})$ of 7 and $40.0 \mu \mathrm{L}(0.019 \mathrm{mmol})$ of KHMDS and $30.0 \mathrm{mg}(0.098 \mathrm{mmol})$ of $\mathbf{4 0}$ were 
reacted for 24 hours. Work-up afforded $16.5 \mathrm{mg}(55 \%)$ of the desired product as colorless oil. $\mathrm{R}_{\mathrm{f}}(1: 1$ hexane to ethyl acetate $)=0.3 ;[\alpha]_{\mathrm{D}}{ }^{23}=-1.8\left(\mathrm{CHCl}_{3}, \mathrm{c}=7 \mathrm{mg} / \mathrm{mL}\right)$; HPLC analysis - Chiralcel AD-H column 90:10 hexanes to isopropanol $1.0 \mathrm{~mL} / \mathrm{min}$. Major enantiomer: 17.3 minutes, Minor enantiomer: 15.9 minutes; ${ }^{1} \mathrm{H}$ NMR (400 MHz), $\delta 7.72$ $(1 \mathrm{H}, \mathrm{d}, J=8.9 \mathrm{~Hz}), 6.35(1 \mathrm{H}, \mathrm{d}, J=8.5 \mathrm{~Hz}), 6.07(1 \mathrm{H}, \mathrm{s}), 4.50(1 \mathrm{H}, \mathrm{dd}, J=5.0,10.9 \mathrm{~Hz})$, $4.2(1 \mathrm{H}, \mathrm{dd}, J=11.0,11.0 \mathrm{~Hz}), 4.15(2 \mathrm{H}, \mathrm{q}, J=7.2 \mathrm{~Hz}), 3.36(4 \mathrm{H}, \mathrm{q}, J=7.2 \mathrm{~Hz}), 3.18(1 \mathrm{H}$, m), $2.91(1 \mathrm{H}, \mathrm{dd}, J=4.5,16.8 \mathrm{~Hz}), 2.33(1 \mathrm{H}, \mathrm{dd}, J=4.5,16.8 \mathrm{~Hz}), 1.25(3 \mathrm{H}, \mathrm{t}, J=7.2 \mathrm{~Hz})$, $1.17(6 \mathrm{H}, \mathrm{t}, J=7.2 \mathrm{~Hz}) ;{ }^{13} \mathrm{C} \mathrm{NMR}\left(100 \mathrm{MHz}, \mathrm{CDCl}_{3}\right) \delta 190.1,171.8,153.2,163.5,129.2$, 108.3, 106.7, 97.2, 70.3, 60.7, 45.0, 41.9, 30.7, 14.1, 12.4; IR (NaCl, $\left.\mathrm{CH}_{2} \mathrm{Cl}_{2}\right)$ 1730, 1665, 1600; HRMS (FAB+) calcd for $\mathrm{C}_{17} \mathrm{H}_{24} \mathrm{NO}_{4}(\mathrm{M}+\mathrm{H})^{+}: 306.1705$. Found 306.1694 .

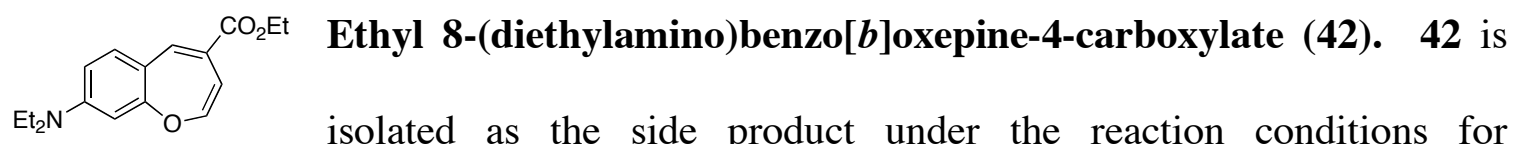

compound 41. ${ }^{1} \mathrm{H}$ NMR $(400 \mathrm{MHz}), \delta 7.46(1 \mathrm{H}, \mathrm{d}, J=15.5 \mathrm{~Hz}), 7.35(1 \mathrm{H}, \mathrm{d}, J=8.5 \mathrm{~Hz})$, $6.79(1 \mathrm{H}, \mathrm{s}), 6.68(1 \mathrm{H}, \mathrm{s}), 6.67(1 \mathrm{H}, \mathrm{d}, J=8.2 \mathrm{~Hz}), 6.37(1 \mathrm{H}, \mathrm{d}, J=15.5 \mathrm{~Hz}), 4.25(2 \mathrm{H}, \mathrm{q}$, $J=7.1 \mathrm{~Hz}), 3.42(4 \mathrm{H}, \mathrm{q}, J=7.1 \mathrm{~Hz}), 1.33(3 \mathrm{H}, \mathrm{t}, J=7.1 \mathrm{~Hz}), 1.20(6 \mathrm{H}, \mathrm{t}, J=7.1 \mathrm{~Hz}) ;{ }^{13} \mathrm{C}$ NMR $\left(100 \mathrm{MHz}, \mathrm{CDCl}_{3}\right) \delta 186.8,141.9,130.9,122.5,105.1,100.1,93.7,66.9,60.9$, 45.2, 14.6, $12.9 \mathrm{~cm}^{-1}$; IR $\left(\mathrm{NaCl}, \mathrm{CH}_{2} \mathrm{Cl}_{2}\right)$ 1702, 1614, 1112; HRMS (FAB+) calcd for $\mathrm{C}_{17} \mathrm{H}_{21} \mathrm{NO}_{3}(\mathrm{M}+\mathrm{H})^{+}:$288.1521. Found 288.1630.

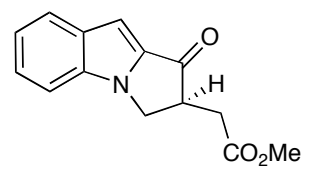

(S)-(1-Oxo-2,3-dihydro-1H-3a-aza-cyclopenta $[a]$ inden-2-yl)acetic acid methyl ester (44). According to the general procedure, $14.0 \mathrm{mg}(0.038 \mathrm{mmol})$ of 6 and $77.0 \mu \mathrm{L}(0.038 \mathrm{mmol})$ of KHMDS and $47.0 \mathrm{mg}(0.192$ 
mmol) of $\mathbf{4 3}$ were reacted for 24 hours. Work-up afforded $37.5 \mathrm{mg}(80 \%)$ of the desired product as colorless oil. $\mathrm{R}_{\mathrm{f}}(1: 1$ hexane to ethyl acetate $)=0.4 ;[\alpha]_{\mathrm{D}}{ }^{23}=-148.7\left(\mathrm{CHCl}_{3}, \mathrm{c}\right.$ $=8 \mathrm{mg} / \mathrm{mL}$ ); HPLC analysis - Chiralcel AD-H 90:10 hexanes to isopropanol $0.5 \mathrm{~mL} / \mathrm{min}$. Minor enantiomer: 28.3 minutes, Major enantiomer: 33.2 minutes; ${ }^{1} \mathrm{H}$ NMR (400 MHz),

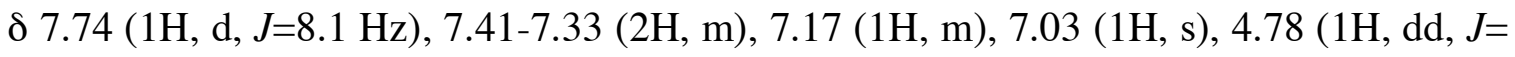
8.3, $11.1 \mathrm{~Hz}), 4.10(1 \mathrm{H}, \mathrm{dd}, J=5.3,11.1 \mathrm{~Hz}), 3.70(3 \mathrm{H}, \mathrm{s}), 3.57(1 \mathrm{H}, \mathrm{m}), 3.11(1 \mathrm{H}, \mathrm{dd}, J=$ 3.6, $17.4 \mathrm{~Hz}), 2.73(1 \mathrm{H}, \mathrm{dd}, J=9.8,17.4 \mathrm{~Hz}) ;{ }^{13} \mathrm{C}\left(100 \mathrm{MHz}, \mathrm{CDCl}_{3}\right) \delta$ 193.2, 171.9, 135.2, 134.7, 132.0, 125.3, 124.2, 121.5, 110.5, 99.6, 52.1, 46.8, 34.3. IR $\left(\mathrm{NaCl}, \mathrm{CH}_{2} \mathrm{Cl}_{2}\right)$ 1731, 1704, 1539, 1315; HRMS (FAB+) calcd for $\mathrm{C}_{14} \mathrm{H}_{14} \mathrm{NO}_{3}(\mathrm{M}+\mathrm{H})^{+}:$244.0974. Found 244.0973.

$(2 \mathrm{R})-(4-O x o-c h r o m a n-3-y l)-a c e t i c$
According to the general procedure, $11.3 \mathrm{mg}(0.024 \mathrm{mmol})$ of ent45 and $48.0 \mu \mathrm{L}(0.024 \mathrm{mmol})$ of KHMDS and $30.0 \mathrm{mg}(0.122 \mathrm{mmol})$ of $\mathbf{4 6}$ were reacted for 60 minutes. Work-up afforded $28.2 \mathrm{mg}$ (94\%) of the desired product as colorless oil. $\mathrm{R}_{\mathrm{f}}(4: 1$ hexane to ethyl acetate $)=0.4 ;[\alpha]_{\mathrm{D}}^{23}=-4.0\left(\mathrm{CHCl}_{3}, \mathrm{c}=10 \mathrm{mg} / \mathrm{mL}\right) ; \mathrm{HPLC}$ analysis - Chiralcel AD-H column 90:10 hexanes to isopropanol $1.0 \mathrm{~mL} / \mathrm{min}$. Major enantiomer: 8.8 minutes, Minor enantiomer: 11.7 minutes; ${ }^{1} \mathrm{H}$ NMR $\left(400 \mathrm{MHz}, \mathrm{CDCl}_{3}\right) \delta$ $7.86(1 \mathrm{H}, \mathrm{dd}, J=1.6,7.9 \mathrm{~Hz}), 7.45(1 \mathrm{H}, \mathrm{m}), 6.99(1 \mathrm{H}, \mathrm{dd}, J=7.4,7.4 \mathrm{~Hz}), 6.94(1 \mathrm{H}, \mathrm{d}, J=$ $8.4 \mathrm{~Hz}), 5.91(1 \mathrm{H}, \mathrm{ddt}, J=5.8,11.0,16.4 \mathrm{~Hz}), 5.32(1 \mathrm{H}, \mathrm{dd}, J=1.3,17.2 \mathrm{~Hz}), 5.23(1 \mathrm{H}$, dd, $J=1.1,11.2 \mathrm{~Hz}), 4.61(2 \mathrm{H}, \mathrm{dd}, J=1.1,6.4 \mathrm{~Hz}), 4.57(1 \mathrm{H}, \mathrm{dd}, J=5.3,11.2 \mathrm{~Hz}), 4.27$ $(1 \mathrm{H}, \mathrm{dd}, J=11.6,11.6 \mathrm{~Hz}), 3.32(1 \mathrm{H}, \mathrm{dddd}, J=5.1,5.1,8.1,13.0 \mathrm{~Hz}), 2.95(1 \mathrm{H}, \mathrm{dd}, J=$ 4.9, $17.0 \mathrm{~Hz}), 2.43(1 \mathrm{H}, \mathrm{dd}, J=8.1,17.0 \mathrm{~Hz}) ;{ }^{13} \mathrm{C} \mathrm{NMR}\left(100 \mathrm{MHz}, \mathrm{CDCl}_{3}\right) \delta 192.5$, 
171.0, 161.7, 136.0, 131.8, 127.3, 121.5, 120.4, 118.6, 117.8, 70.1, 65.6, 42.4, 30.2; IR $\left(\mathrm{NaCl}, \mathrm{CH}_{2} \mathrm{Cl}_{2}\right) 1738,1690,1612,1474 \mathrm{~cm}^{-1}$; HRMS (FAB+) calcd for $\mathrm{C}_{14} \mathrm{H}_{15} \mathrm{O}_{4}(\mathrm{M}+\mathrm{H})^{+}$: 247.0892, Found 247.0975.

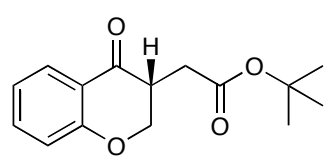

(2R)-(4-Oxo-chroman-3-yl)-acetic acid tert-butyl ester (49)

According to the general procedure, $9.0 \mathrm{mg}(0.022 \mathrm{mmol})$ of ent $\mathbf{4 5}$ and $45.0 \mu \mathrm{L}(0.022 \mathrm{mmol})$ of KHMDS and $30.0 \mathrm{mg}(0.114 \mathrm{mmol})$ of $\mathbf{4 8}$ were reacted for 60 minutes. Work-up afforded $28.2 \mathrm{mg}(94 \%)$ of the desired product as colorless oil. $\mathbf{R}_{\mathrm{f}}$ $(4: 1$ hexane to ethyl acetate $)=0.4 ;[\alpha]_{\mathrm{D}}^{23}=-2.5\left(\mathrm{CHCl}_{3}, c=20 \mathrm{mg} / \mathrm{mL}\right) ; \mathrm{HPLC}$ analysis - Chiralcel AD-H column 90:10 hexanes to isopropanol $1.0 \mathrm{~mL} / \mathrm{min}$. major enantiomer 5.64 minutes, minor enantiomer 5.96 minutes; ${ }^{1} \mathrm{H}$ NMR $\left(400 \mathrm{MHz}, \mathrm{CDCl}_{3}\right) \delta 7.86(1 \mathrm{H}, \mathrm{d}$, $J=7.9 \mathrm{~Hz}), 7.44(1 \mathrm{H}, \mathrm{m}), 6.99(1 \mathrm{H}, \mathrm{dd}, J=7.9,7.9 \mathrm{~Hz}), 6.94(1 \mathrm{H}, \mathrm{d}, J=8.3 \mathrm{~Hz}), 4.54(1 \mathrm{H}$, dd, $J=5.3,11.3 \mathrm{~Hz}), 4.26(1 \mathrm{H}, \mathrm{dd}, J=11.5,11.5 \mathrm{~Hz}), 3.25(1 \mathrm{H}, \mathrm{dddd}, J=4.9,12.8,17.0$, $17.0 \mathrm{~Hz}), 2.82(1 \mathrm{H}, \mathrm{dd}, J=4.7,17.0 \mathrm{~Hz}), 2.35(1 \mathrm{H}, \mathrm{dd}, J=8.5,17.0 \mathrm{~Hz}), 1.44(9 \mathrm{H}, \mathrm{s}) ;{ }^{13} \mathrm{C}$ NMR (100 MHz, $\left.\mathrm{CDCl}_{3}\right) \delta 192.7,170.5,161.6,135.9,127.3,121.4,120.5,117.7,81.2$, 70.2, 42.6, 31.5, 28.0; IR ( $\left.\mathrm{NaCl}, \mathrm{CH}_{2} \mathrm{Cl}_{2}\right)$ 1730, 1694, 1607, $1476 \mathrm{~cm}^{-1}$; HRMS (FAB+) calcd for $\mathrm{C}_{15} \mathrm{H}_{19} \mathrm{O}_{4}(\mathrm{M}+\mathrm{H})^{+}$: 263.1283, Found 263.1287.

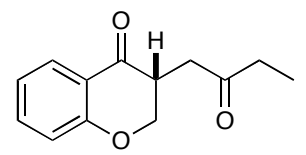

(2R)-(3-(2-Oxo-pentyl)-chroman-4-one (53) According to the general procedure, $9.0 \mathrm{mg}(0.019 \mathrm{mmol})$ of ent $-\mathbf{4 5}$ and $37.0 \mu \mathrm{L}(0.019$ mmol) of KHMDS and $20.0 \mathrm{mg}(0.0916 \mathrm{mmol})$ of $\mathbf{5 2}$ were reacted for 60 minutes. Workup afforded $19.0 \mathrm{mg}(94 \%)$ of the desired product as colorless oil. $\mathrm{R}_{\mathrm{f}}(4: 1$ hexane to ethyl acetate $)=0.4 ;[\alpha]_{D}^{23}=-6.3\left(\mathrm{CHCl}_{3}, \mathrm{c}=8 \mathrm{mg} / \mathrm{mL}\right) ;$ HPLC analysis - Chiralcel AD-H 
column 90:10 hexanes to isopropanol $0.5 \mathrm{~mL} / \mathrm{min}$. Minor enantiomer: 18.62 minutes, Major enantiomer: 19.41 minutes; ${ }^{1} \mathrm{H}$ NMR $\left(400 \mathrm{MHz}, \mathrm{CDCl}_{3}\right) \delta 7.84(1 \mathrm{H}, \mathrm{d}, J=7.6 \mathrm{~Hz})$, $7.45(1 \mathrm{H}, \mathrm{m}), 6.99(1 \mathrm{H}, \mathrm{dd}, J=7.5,7.6 \mathrm{~Hz}), 6.94(1 \mathrm{H}, \mathrm{d}, J=8.3 \mathrm{~Hz}), 4.51(1 \mathrm{H}, \mathrm{dd}, J=5.3$, $11.0 \mathrm{~Hz}), 4.21(1 \mathrm{H}, \mathrm{dd}, J=11.5,11.5 \mathrm{~Hz}), 3.40(1 \mathrm{H}, \mathrm{dddd}, J=4.9,12.8,17.0,17.0 \mathrm{~Hz})$, $2.99(1 \mathrm{H}, \mathrm{dd}, J=4.6,17.9 \mathrm{~Hz}), 2.61-2.41(3 \mathrm{H}, \mathrm{m}), 1.08(3 \mathrm{H}, \mathrm{t}, J=7.2 \mathrm{~Hz}) ;{ }^{13} \mathrm{C}$ NMR $(100$ $\left.\mathrm{MHz}, \mathrm{CDCl}_{3}\right) \delta 208.3,193.4,161.7,135.9,127.2,121.4,120.5,117.8,70.2,41.7,37.6$, 36.2, 7.7; IR ( $\left.\mathrm{NaCl}, \mathrm{CH}_{2} \mathrm{Cl}_{2}\right) 1715,1687,1605,1437 \mathrm{~cm}^{-1}$; HRMS (FAB+) calcd for $\mathrm{C}_{13} \mathrm{H}_{15} \mathrm{O}_{3}(\mathrm{M}+\mathrm{H})^{+}:$219.1021, Found 219.1026.

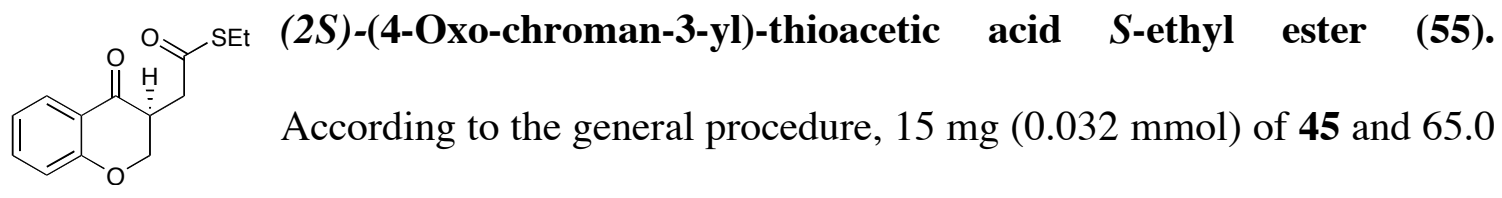
$\mu \mathrm{L}(0.032 \mathrm{mmol})$ of KHMDS and $30.0 \mathrm{mg}(0.159 \mathrm{mmol})$ of $\mathbf{5 4}$ were reacted for 2 hours. Work-up afforded $27.0 \mathrm{mg}(90 \%)$ of the desired product as colorless oil. $\mathrm{R}_{\mathrm{f}}(1: 1$ hexane to ethyl acetate $)=0.7 ;[\alpha]_{\mathrm{D}}^{23}=+1.25\left(\mathrm{CHCl}_{3}, 10 \mathrm{mg} / \mathrm{mL}\right) ;$ HPLC analysis-Chiralcel AD-H column 90:10 hexanes to isopropanol $1.0 \mathrm{~mL} / \mathrm{min}$. Minor enantiomer: 8.1 minutes, Major enantiomer: 9.0 minutes; ${ }^{1} \mathrm{H}$ NMR $\left(400 \mathrm{MHz}, \mathrm{CDCl}_{3}\right) \delta 7.88(1 \mathrm{H}, \mathrm{m}), 7.47(1 \mathrm{H}$, m), 7.04-6.95 (2H, m), $4.58(1 \mathrm{H}, \mathrm{dd}, J=5.1,11.3 \mathrm{~Hz}), 4.25(1 \mathrm{H}, \mathrm{dd}, J=11.3,11.3 \mathrm{~Hz})$, $3.38(1 \mathrm{H}, \mathrm{m}), 3.21(1 \mathrm{H}, \mathrm{dd}, J=4.4,16.5 \mathrm{~Hz}), 2.92(2 \mathrm{H}, \mathrm{q}, J=7.3 \mathrm{~Hz}), 2.64(1 \mathrm{H}, \mathrm{dd}, J=$ 8.8, $16.1 \mathrm{~Hz}), 1.27(3 \mathrm{H}, \mathrm{t}, J=7.3 \mathrm{~Hz}) ;{ }^{13} \mathrm{C} \mathrm{NMR}\left(100 \mathrm{MHz}, \mathrm{CDCl}_{3}\right) \delta 196.8,192.0,161.5$, 135.9, 127.3, 121.4, 120.3, 117.7, 70.0, 42.7, 39.4, 23.6, 14.7; IR $\left(\mathrm{NaCl}, \mathrm{CH}_{2} \mathrm{Cl}_{2}\right)$ 1680, 1607, $1476 \mathrm{~cm}^{-1}$; HRMS $(\mathrm{FAB}+)$ calcd for $\mathrm{C}_{13} \mathrm{H}_{15} \mathrm{O}_{3} \mathrm{~S}(\mathrm{M}+\mathrm{H})^{+}$: 251.0742, Found 251.0730. 


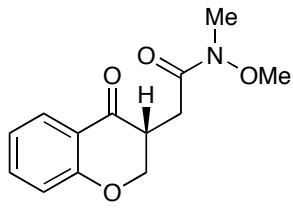

(57). According to the general procedure, $18.7 \mathrm{mg}(0.040 \mathrm{mmol})$ of ent-45 and $80.0 \mu \mathrm{L}(0.040 \mathrm{mmol})$ of KHMDS and $50.0 \mathrm{mg}(0.200$ mmol) of 56 were reacted for 8 hours. Work-up afforded $47.0 \mathrm{mg}(94 \%)$ of the desired product as colorless oil. $\mathrm{R}_{\mathrm{f}}(1: 1$ hexane to ethyl acetate $)=0.3 ;[\alpha]_{\mathrm{D}}^{23}=-12.9^{\circ}\left(\mathrm{CHCl}_{3}, \mathrm{c}\right.$ $=10 \mathrm{mg} / \mathrm{mL}$ ); GC analysis-Chiraldex AD-H column 90:10 hexanes to isopropanol 1.0 $\mathrm{mL} / \mathrm{min}$. Major enantiomer: 16.0 minutes, Minor enantiomer: 19.8 minutes; ${ }^{1} \mathrm{H}$ NMR $\left(400 \mathrm{MHz}, \mathrm{CDCl}_{3}\right) \delta 7.85(1 \mathrm{H}, \mathrm{dd}, J=1.42,7.9 \mathrm{~Hz}), 7.44(1 \mathrm{H}, \mathrm{m}), 6.98(1 \mathrm{H}, \mathrm{dd}, J=7.6$, $7.6 \mathrm{~Hz}), 6.93(1 \mathrm{H}, \mathrm{d}, J=8.4 \mathrm{~Hz}), 4.58(1 \mathrm{H}, \mathrm{dd}, J=5.3,11.3 \mathrm{~Hz}), 4.28(1 \mathrm{H}, \mathrm{dd}, J=11.3$, $11.3 \mathrm{~Hz}), 3.70(3 \mathrm{H}, \mathrm{s}), 3.39(1 \mathrm{H}, \mathrm{dddd}, J=4.7,4.7,9.0,12.8 \mathrm{~Hz}), 3.18(3 \mathrm{H}, \mathrm{s}), 3.06(1 \mathrm{H}$, $\mathrm{dd}, J=4.0,17.2 \mathrm{~Hz}), 2.50(1 \mathrm{H}, \mathrm{dd}, J=8.3,17.2 \mathrm{~Hz}) ;{ }^{13} \mathrm{C} \mathrm{NMR}\left(100 \mathrm{MHz}, \mathrm{CDCl}_{3}\right) \delta$ $193.5,161.7,135.8,127.2,121.3,120.6,117.7,70.5,61.2,42.0,32.2,27.9 ; \mathrm{IR}(\mathrm{NaCl}$, $\left.\mathrm{CH}_{2} \mathrm{Cl}_{2}\right)$ 1694, 1658, 1600, $1476 \mathrm{~cm}^{-1}$. HRMS (FAB+) calcd for $\mathrm{C}_{13} \mathrm{H}_{16} \mathrm{NO}_{4}(\mathrm{M}+\mathrm{H})^{+}$: 250.1079, Found 250.1067.

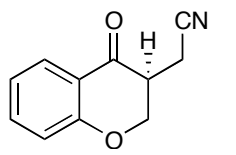

(2S)-(4-Oxo-chroman-3-yl)-acetonitrile (59). According to the general procedure, $15.0 \mathrm{mg}(0.032 \mathrm{mmol})$ of 45 and $64.0 \mu \mathrm{L}(0.032 \mathrm{mmol})$ of KHMDS and $30.0 \mathrm{mg}(0.160 \mathrm{mmol})$ of $\mathbf{5 8}$ were reacted for 24 hours. Work-up afforded $24.0 \mathrm{mg}(88 \%)$ of the desired product as colorless oil. $\mathrm{R}_{\mathrm{f}}(1: 1$ hexane to ethyl acetate $)=$ $0.7 ;[\alpha]_{\mathrm{D}}^{23}=+7.0\left(\mathrm{CHCl}_{3}, \mathrm{c}=10 \mathrm{mg} / \mathrm{mL}\right) ;$ HPLC analysis-Chiralcel AD-H column 90:10 hexanes to isopropanol $1.0 \mathrm{~mL} / \mathrm{min}$. Minor enantiomer: 14.8 minutes, Major enantiomer: 15.6 minutes; ${ }^{1} \mathrm{H}$ NMR $\left(400 \mathrm{MHz} \mathrm{CDCl}_{3}\right) \delta 7.87(1 \mathrm{H}, \mathrm{m}), 7.50(1 \mathrm{H}, \mathrm{m})$, $7.04(1 \mathrm{H}, \mathrm{m}), 6.98(1 \mathrm{H}, \mathrm{m}), 4.72(1 \mathrm{H}, \mathrm{dd}, J=5.1,11.5 \mathrm{~Hz}), 4.32(1 \mathrm{H}, \mathrm{dd}, J=11.5,11.7$ 
Hz), $3.17(1 \mathrm{H}$, dddd, $J=4.9,9.4,13.8,13.8 \mathrm{~Hz}), 2.97(1 \mathrm{H}, \mathrm{dd}, J=4.7,17.5 \mathrm{~Hz}), 2.58(1 \mathrm{H}$, $\mathrm{dd}, J=9.2,17.5 \mathrm{~Hz}) ;{ }^{13} \mathrm{C} \mathrm{NMR}\left(100 \mathrm{MHz}, \mathrm{CDCl}_{3}\right) \delta 190.0,161.6,136.7,127.4,122.0$, 119.8, 118.0, 117.0, 69.4, 42.0, 14.1; IR $\left(\mathrm{NaCl}, \mathrm{CH}_{2} \mathrm{Cl}_{2}\right)$ 2247, 1687, 1607, $1287 \mathrm{~cm}^{-1}$; HRMS (FAB+) calcd for $\mathrm{C}_{11} \mathrm{H}_{10} \mathrm{NO}_{2}(\mathrm{M}+\mathrm{H})^{+}$: 188.0712, Found 188.0719.

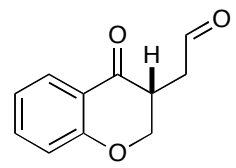

(2R)-(4-Oxo-chroman-3-yl)-acetaldehyde (62). According to the general procedure, $20.0 \mathrm{mg}(0.042 \mathrm{mmol})$ of $\mathbf{4 5}$ and $84.0 \mu \mathrm{L}(0.042$ $\mathrm{mmol})$ of KHMDS and $40.0 \mathrm{mg}(0.210 \mathrm{mmol})$ of $\mathbf{6 1}$ were reacted for 24 hours. Work-up afforded $20.0 \mathrm{mg}(50 \%)$ of the desired product as colorless oil. $\mathrm{R}_{\mathrm{f}}(1: 1$ hexane to ethyl acetate $)=0.7 ;[\alpha]_{\mathrm{D}}^{23}=-12.9\left(\mathrm{CHCl}_{3}, \mathrm{c}=10 \mathrm{mg} / \mathrm{mL}\right) ; \mathrm{HPLC}$ analysis - Chiralcel AD-H column 90:10 hexanes to isopropanol $1.0 \mathrm{~mL} / \mathrm{min}$. Major enantiomer: 12.0 minutes. Minor enantiomer: 12.7 minutes; ${ }^{1} \mathrm{H}$ NMR $\left(400 \mathrm{MHz}, \mathrm{CDCl}_{3}\right) \delta 9.86(1 \mathrm{H}, \mathrm{s}), 7.86(1 \mathrm{H}$, m), $7.46(1 \mathrm{H}, \mathrm{m}), 7.10(1 \mathrm{H}, \mathrm{m}), 6.95(1 \mathrm{H}, \mathrm{m}), 4.53(1 \mathrm{H}, \mathrm{dd}, J=5.3,11.3 \mathrm{~Hz}), 4.22(1 \mathrm{H}$, dd, $J=11.5,11.7 \mathrm{~Hz}), 3.43(1 \mathrm{H}, \mathrm{dddd}, J=5.1,7.0,12.4,12.4 \mathrm{~Hz}), 3.06(1 \mathrm{H}, \mathrm{dd}, J=5.1$, $18.5 \mathrm{~Hz}), 2.53(1 \mathrm{H}, \mathrm{dd}, J=7.2,18.5 \mathrm{~Hz}) ;{ }^{13} \mathrm{C} \mathrm{NMR}\left(100 \mathrm{MHz}, \mathrm{CDCl}_{3}\right) \delta, 199.1,192.8$, 161.9, 136.5, 127.4, 121.7, 120.5, 118.1, 70.2, 40.7, 39.4; IR (NaCl, $\left.\mathrm{CH}_{2} \mathrm{Cl}_{2}\right)$ 1726, 1690, $1600,1474 \mathrm{~cm}^{-1}$. Compound unstable to mass spectroscopy.

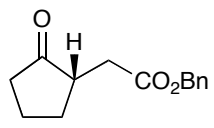

(2S)-(2-Oxo-cyclopentyl)-acetic acid benzyl ester (64). According to the general procedure, $15.6 \mathrm{mg}(0.043 \mathrm{mmol})$ of 6 and $86.0 \mu \mathrm{L}(0.043 \mathrm{mmol})$ of KHMDS and $50.0 \mathrm{mg}(0.215 \mathrm{mmol})$ of $\mathbf{6 3}$ were reacted for 24 hours. Work-up afforded $42.2 \mathrm{mg}(85 \%)$ of the desired product as colorless oil. $\mathrm{R}_{\mathrm{f}}$ (1:1 hexane to ethyl acetate $)=0.7 ;[\alpha]_{\mathrm{D}}^{23}=-96.0\left(\mathrm{CHCl}_{3}, \mathrm{c}=10 \mathrm{mg} / \mathrm{mL}\right) ;$ HPLC analysis-Chiralcel AD-H 
column 90:10 hexanes to isopropanol $0.5 \mathrm{~mL} / \mathrm{min}$. Major enantiomer: 16.1 minutes. Minor enantiomer: 18.1 minutes; ${ }^{1} \mathrm{H}$ NMR (400 MHz, $\left.\mathrm{CDCl}_{3}\right) \delta$ 7.36-7.23 (5H, m), 5.09 (2H, s), $2.76(1 \mathrm{H}, \mathrm{ddd}, J=7.6,19.6,22.1 \mathrm{~Hz}), 2.48-2.42(2 \mathrm{H}, \mathrm{m}), 2.35-2.22(2 \mathrm{H}, \mathrm{m}), 2.12$ $(1 \mathrm{H}, \mathrm{m}), 2.01(1 \mathrm{H}, \mathrm{m}), 1.76(1 \mathrm{H}, \mathrm{m}), 1.58(1 \mathrm{H}, \mathrm{m}) ;{ }^{13} \mathrm{C} \mathrm{NMR}\left(100 \mathrm{MHz}, \mathrm{CDCl}_{3}\right) \delta 219.1$, $171.9,135.6,128.5,128.2,128.1,66.4,45.5,37.3,33.8,29.2,20.5 ; \mathrm{IR}\left(\mathrm{NaCl}, \mathrm{CH}_{2} \mathrm{Cl}_{2}\right)$ 1730, 1454, $1265 \mathrm{~cm}^{-1}$; HRMS $(\mathrm{FAB}+)$ calcd for $\mathrm{C}_{14} \mathrm{H}_{17} \mathrm{O}_{3}(\mathrm{M}+\mathrm{H})^{+}:$233.1178, Found 233.1178 .

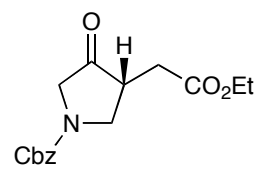

(2R)-(3-Ethoxycarbonylmethyl-4-oxo-pyrrolidine-1-carboxylic acid

benzyl ester (66). According to the general procedure, $12.0 \mathrm{mg}(0.032$ $\mathrm{mmol})$ of 6 and $65.0 \mu \mathrm{L}(0.032 \mathrm{mmol})$ of KHMDS and $50.0 \mathrm{mg}(0.164 \mathrm{mmol})$ of $\mathbf{6 5}$ were reacted for 24 hours. Work-up afforded $40.0 \mathrm{mg}(80 \%)$ of the desired product as colorless oil. $\mathrm{R}_{\mathrm{f}}(1: 1$ hexane to ethyl acetate $\left.)=0.5 ;[\alpha]_{\mathrm{D}}^{23}=+23.1 \mathrm{CHCl}_{3}, \mathrm{c}=10 \mathrm{mg} / \mathrm{mL}\right) ; \mathrm{GC}$ analysis -B-DM column $80{ }^{\circ} \mathrm{C}$ for 120 minutes then ramp to $120{ }^{\circ} \mathrm{C}$ over 10 minutes. Minor enantiomer 144.0 minutes, Major enantiomer 148.7 minutes; ${ }^{1} \mathrm{H}$ NMR $(300 \mathrm{MHz}$, $\left.\mathrm{C}_{6} \mathrm{D}_{5} \mathrm{CD}_{3}\left(80{ }^{\circ} \mathrm{C}\right)\right) \delta$ 7.24-7.10 (5H, m), $5.09(2 \mathrm{H}, \mathrm{s}), 3.92-3.80(4 \mathrm{H}, \mathrm{m}), 3.58(1 \mathrm{H}, \mathrm{dd}, J=$ 18.9, $18.9 \mathrm{~Hz}), 3.42(1 \mathrm{H}, \mathrm{dd}, J=18.8,18.8 \mathrm{~Hz}), 2.98(1 \mathrm{H}, \mathrm{dd}, J=10.5,9 \mathrm{~Hz}), 2.34-2.13$ $(2 \mathrm{H}, \mathrm{m}), 0.98(3 \mathrm{H}, \mathrm{t}, 7.2 \mathrm{~Hz}) ;{ }^{13} \mathrm{C} \mathrm{NMR}\left(100 \mathrm{MHz}, \mathrm{CDCl}_{3}\right) \delta 211.1,171.1,169.0,136.5$, $128.8,128.4,128.3,67.5,61.4,52.7,48.3,44.0,33.0,14.3 ; \mathrm{IR}\left(\mathrm{NaCl}, \mathrm{CH}_{2} \mathrm{Cl}_{2}\right)$ 1760, 1709, $1418 \mathrm{~cm}^{-1}$; HRMS $(\mathrm{FAB}+)$ calcd for $\mathrm{C}_{16} \mathrm{H}_{20} \mathrm{NO}_{5}(\mathrm{M}+\mathrm{H})^{+}:$306.1341, Found 306.1333 . 


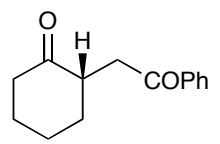

(2S)- 2-(2-Oxo-2-phenyl-ethyl)-cyclohexanone (70). According to the

general procedure, $17.0 \mathrm{mg}(0.046 \mathrm{mmol})$ of 6 and $92.0 \mu \mathrm{L}(0.046 \mathrm{mmol})$ of KHMDS and $50.0 \mathrm{mg}(0.231 \mathrm{mmol})$ of $\mathbf{6 9}$ were reacted for 24 hours. Work-up afforded $30.0 \mathrm{mg}(60 \%)$ of the desired product as colorless oil. $\mathrm{R}_{\mathrm{f}}$ (4:1 hexane to ethyl acetate $)=0.4 ;[\alpha]_{\mathrm{D}}^{23}=-24.3\left(\mathrm{CHCl}_{3}, 20 \mathrm{mg} / \mathrm{mL}\right) ;$ HPLC analysis-Chiralcel OD-H column 95:5 hexanes to isopropanol $0.5 \mathrm{ml} / \mathrm{min}$. Major enantiomer: 10.94 minutes, Minor enantiomer: 13.97 minutes; ${ }^{1} \mathrm{H}$ NMR $\left(400 \mathrm{MHz}, \mathrm{CDCl}_{3}\right) \delta 7.96(2 \mathrm{H}, \mathrm{m}), 7.53(1 \mathrm{H}$, m), $7.43(2 \mathrm{H}, \mathrm{m}), 3.75(1 \mathrm{H}, \mathrm{dd}, J=6.6,17.7 \mathrm{~Hz}), 3.14(1 \mathrm{H}$, dddd, $J=5.9,12.1,12.1,18.5$ $\mathrm{Hz}), 2.65(1 \mathrm{H}, \mathrm{dd}, J=5.8,17.7 \mathrm{~Hz}), 2.43-2.39(2 \mathrm{H}, \mathrm{m}), 2.20-2.08(2 \mathrm{H}, \mathrm{m}), 1.89-1.60(3 \mathrm{H}$, m), $1.42(1 \mathrm{H}, \mathrm{m}) ;{ }^{13} \mathrm{C}$ NMR $\left(100 \mathrm{MHz}, \mathrm{CDCl}_{3}\right) \delta$ 211.5, 198.6, 136.9, 132.9, 128.4, 128.0, 46.4, 41.9, 38.3, 34.2, 27.9, 25.3; IR $\left(\mathrm{NaCl}, \mathrm{CH}_{2} \mathrm{Cl}_{2}\right)$ 1712, 1684, $1441 \mathrm{~cm}^{-1}$; HRMS (FAB+) calcd for $\mathrm{C}_{14} \mathrm{H}_{17} \mathrm{O}_{2}(\mathrm{M}+\mathrm{H})^{+}:$217.1229, Found 217.1226.

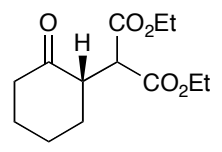

(2S)-2-(2-Oxo-cyclohexyl)-malonic acid diethyl ester (72). According to the general procedure, $14.1 \mathrm{mg}(0.039 \mathrm{mmol})$ of 6 and $78.0 \mu \mathrm{L}(0.039$ mmol) of KHMDS and $50.0 \mathrm{mg}(0.195 \mathrm{mmol})$ of $\mathbf{7 1}$ were reacted for 24 hours. Work-up afforded $49.0 \mathrm{mg}(97 \%)$ of the desired product as colorless oil. $\mathrm{R}_{\mathrm{f}}$ (4:1 hexane to ethyl acetate $)=0.4 ;[\alpha]_{\mathrm{D}}{ }^{23}=-41.8\left(\mathrm{CHCl}_{3}, \mathrm{c}=18 \mathrm{mg} / \mathrm{mL}\right) ; \mathrm{GC}$ analysis; Chiraldex GTA column $130{ }^{\circ} \mathrm{C} 3.0 \mathrm{~mL} / \mathrm{min}$. Major enantiomer: 37.7 minutes. Minor enantiomer: 39.7 minutes; ${ }^{1} \mathrm{H}$ NMR $\left(400 \mathrm{MHz}, \mathrm{CDCl}_{3}\right) \delta 4.16(2 \mathrm{H}, \mathrm{q}, J=7.2 \mathrm{~Hz}), 4.16(2 \mathrm{H}, \mathrm{q}, J=7.2 \mathrm{~Hz})$ $3.61(1 \mathrm{H}, \mathrm{d}, J=9.6 \mathrm{~Hz}), 3.16(1 \mathrm{H}, \mathrm{ddd}, J=5.5,9.6,13.2 \mathrm{~Hz}), 2.41-2.37(2 \mathrm{H}, \mathrm{m}), 2.11-1.99$ $(2 \mathrm{H}, \mathrm{m}), 1.88(1 \mathrm{H}, \mathrm{m}), 1.74-1.46(3 \mathrm{H}, \mathrm{m}), 1.23(3 \mathrm{H}, \mathrm{t}, J=7.2 \mathrm{~Hz}), 1.23(3 \mathrm{H}, \mathrm{t}, J=7.2$ $\mathrm{Hz}) ;{ }^{13} \mathrm{C}$ NMR $\left(100 \mathrm{MHz}, \mathrm{CDCl}_{3}\right) \delta$ 209.7, 168.5, 168.3, 61.4, 52.2, 50.2, 41.8, 31.1, 
27.7, 25.0, 14.0, 13.9; IR ( $\left.\mathrm{NaCl}, \mathrm{CH}_{2} \mathrm{Cl}_{2}\right)$ 1750, 1728, $1452 \mathrm{~cm}^{-1}$; HRMS (FAB+) calcd for $\mathrm{C}_{13} \mathrm{H}_{21} \mathrm{O}_{5}(\mathrm{M}+\mathrm{H})^{+}$: 257.1389, Found 257.1399. 


\section{Assignment of Absolute Stereochemistry:}

Absolute stereochemistry for compounds $\mathbf{3}$ and $\mathbf{1 7}$ formed with catalyst $\mathbf{6}$ were determined to be $S$ by comparison of the measured optical rotation value with the corresponding literature data. ${ }^{4,5}$ Absolute stereochemistry for compound $\mathbf{1 9}$ and 49 formed with catalyst $\mathbf{6}$ were determined to be $S$ by conversion to compound $\mathbf{3}$ and comparison of HPLC data (Scheme 1).

\section{Scheme 1.}

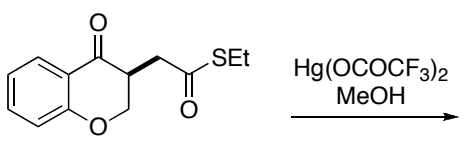

19

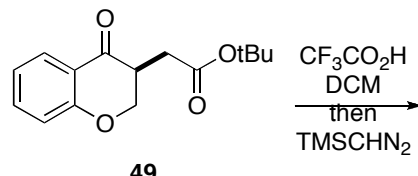

49
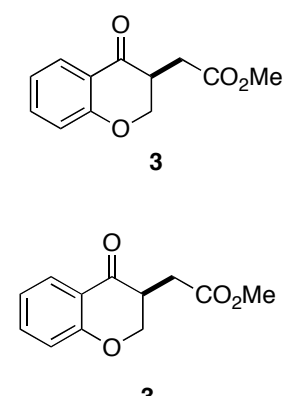

The following Scheme summarizes our assigned stereochemistry for other adducts by analogy and notes the similarity of absolute sign (optical rotation) as well as retention on HPLC using common columns. AD and AD-H columns are identical except for particle size. Sixteen structurally related compounds are self-consistent with regard to sign of optical rotation, retention on the chiral column and assigned major enantiomer in the asymmetric Stetter reaction. This is provided as corroborating evidence for our assignment by analogy.

\section{Scheme 2.}




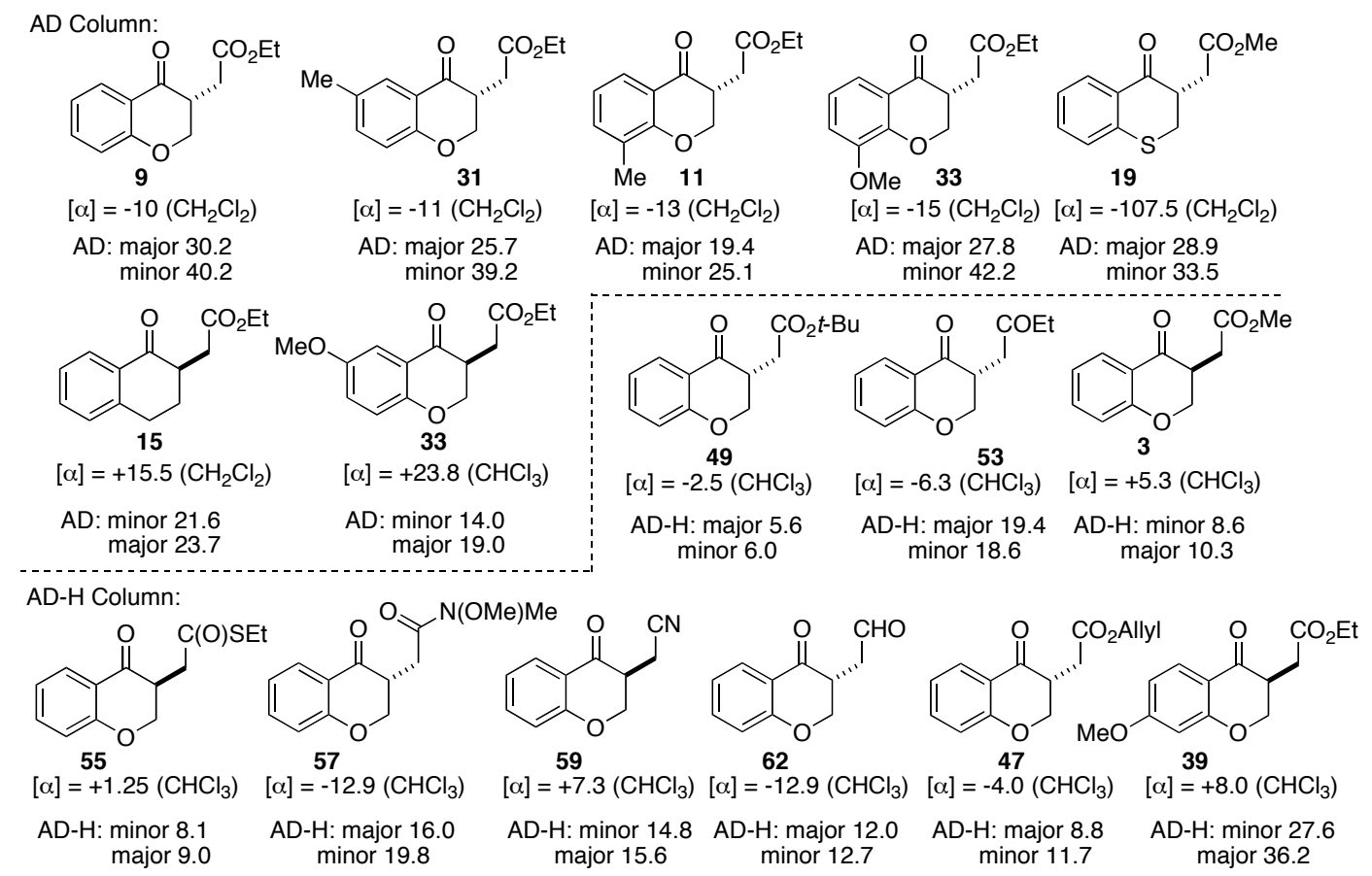

The following Scheme illustrates the six compounds which do not fit the above model in terms of both rotation sign and HPLC retention order for major enantiomer. We note that in a number of cases, either rotation sign $(\mathbf{2 1}, \mathbf{2 3}, \mathbf{3 5}, \mathbf{3 7})$ or HPLC retention order (41) are consistent with the structures above. Compounds 21, 35 and $\mathbf{1 3}$ form too small a subset for meaningful comparison (separation on OD-H was only observed for these three compounds). Compound $\mathbf{1 3}$ does not fit in terms of rotation sign and is part of the small subset of OD-H compounds; we note, however, that it seems highly unlikely that this compound would form the opposite enantiomer in the Stetter reaction when the phenyl ketone is sterically very similar to the Weinreb amide substrate, which we show above provides the major enantiomer consistent with our assignment.

\section{Scheme 3}



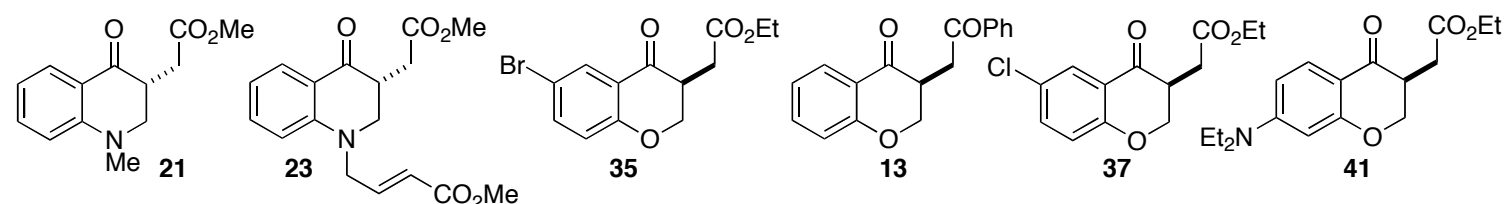

$[\alpha]=-46.6\left(\mathrm{CH}_{2} \mathrm{Cl}_{2}\right)[\alpha]=-20.8\left(\mathrm{CH}_{2} \mathrm{Cl}_{2}\right)$

$[\alpha]=+10.2\left(\mathrm{CHCl}_{3}\right)[\alpha]=-36.2\left(\mathrm{CHCl}_{3}\right)$

$[\alpha]=+1.4\left(\mathrm{CHCl}_{3}\right)$

$[\alpha]=-1.8\left(\mathrm{CHCl}_{3}\right)$

OD-H: major $29.6 \quad$ AD: minor 42.3

OD-H: major 24.6 OD-H: major 10.9

minor 26.3

minor 13.9

AD: major 10.9

AD-H: minor 15.9 major 17.3 
(3)

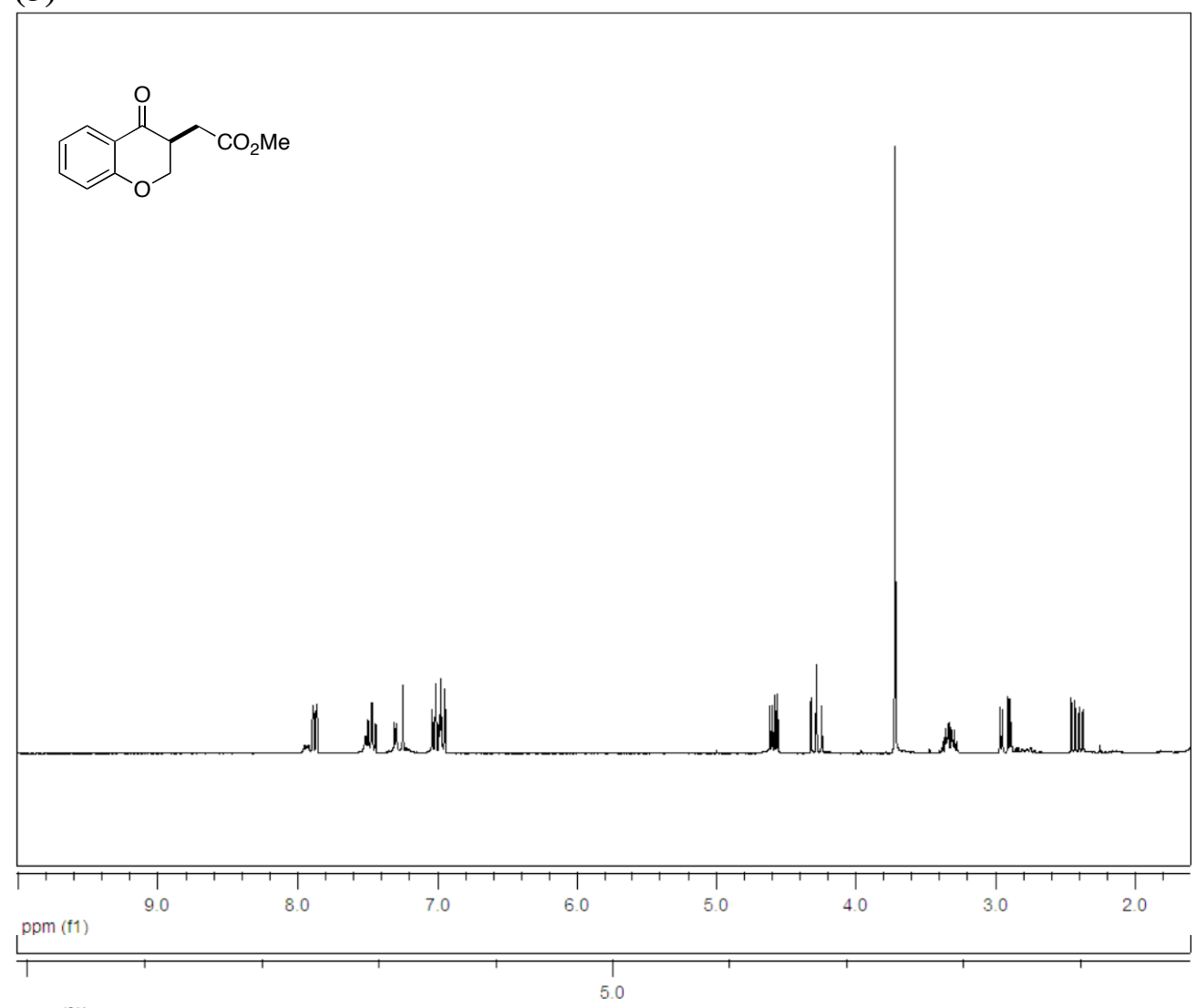

(9)

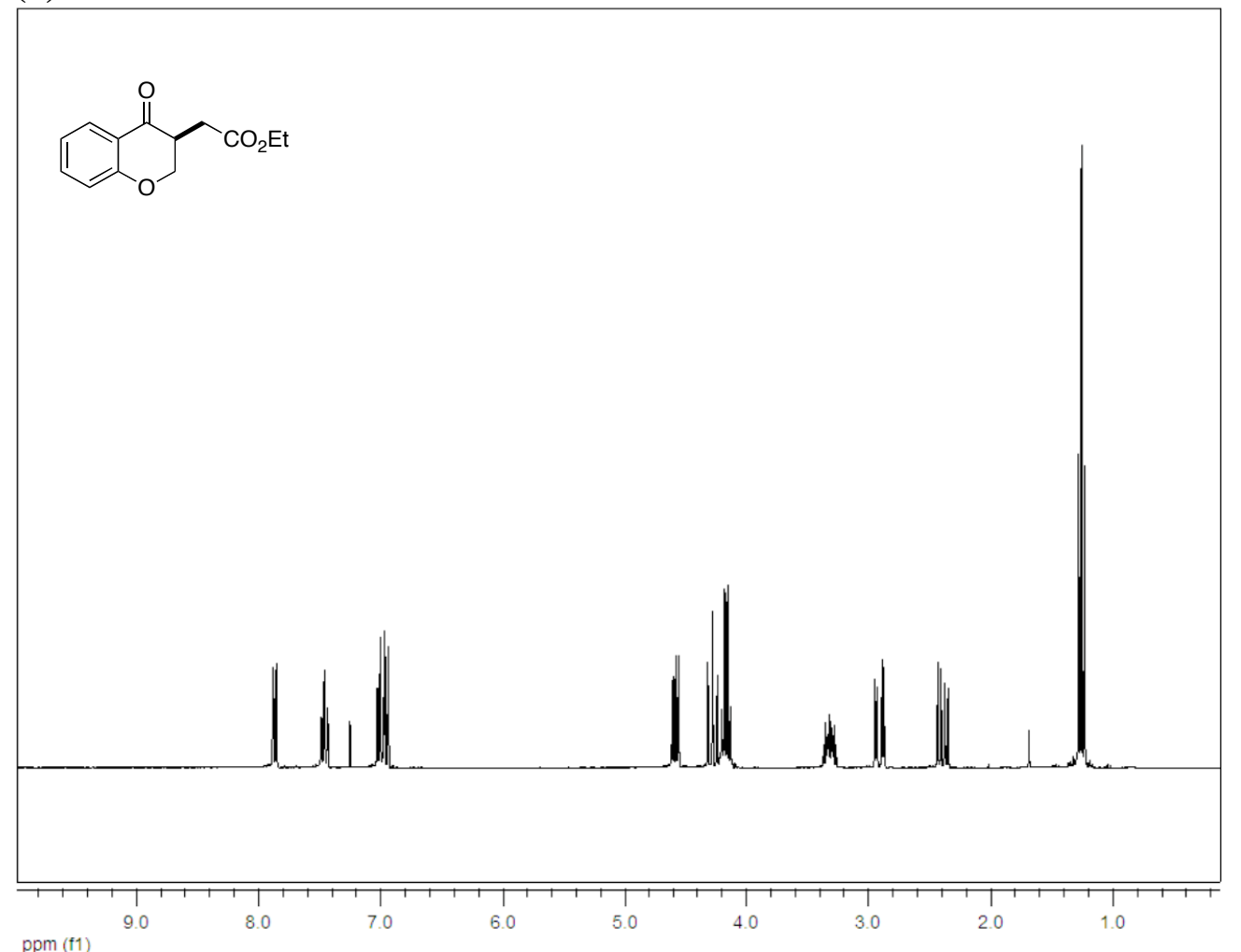


(11)

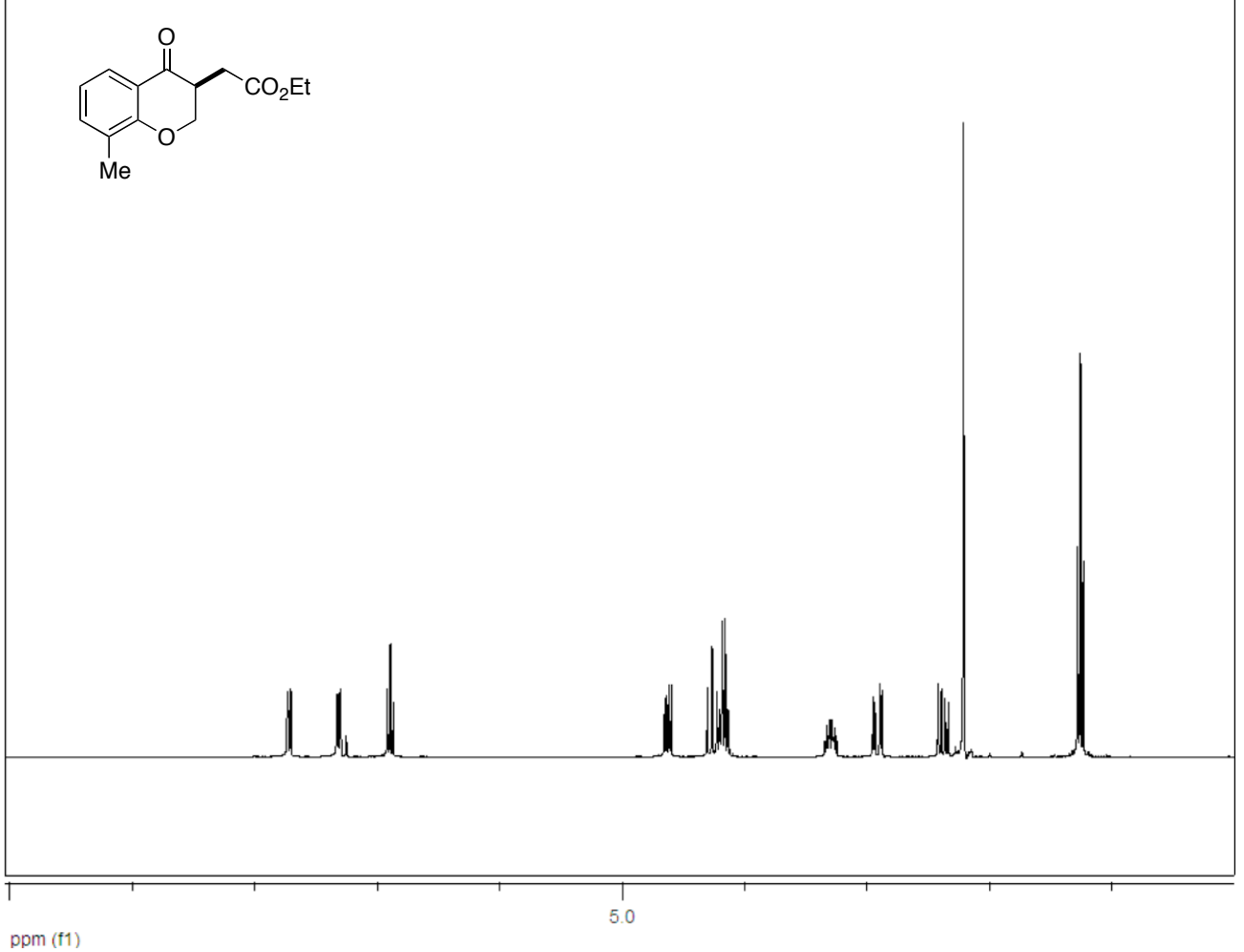


(13)<smiles>O=C(CC1(CC(=O)c2ccccc2)COc2ccccc2C1=O)c1ccccc1</smiles>
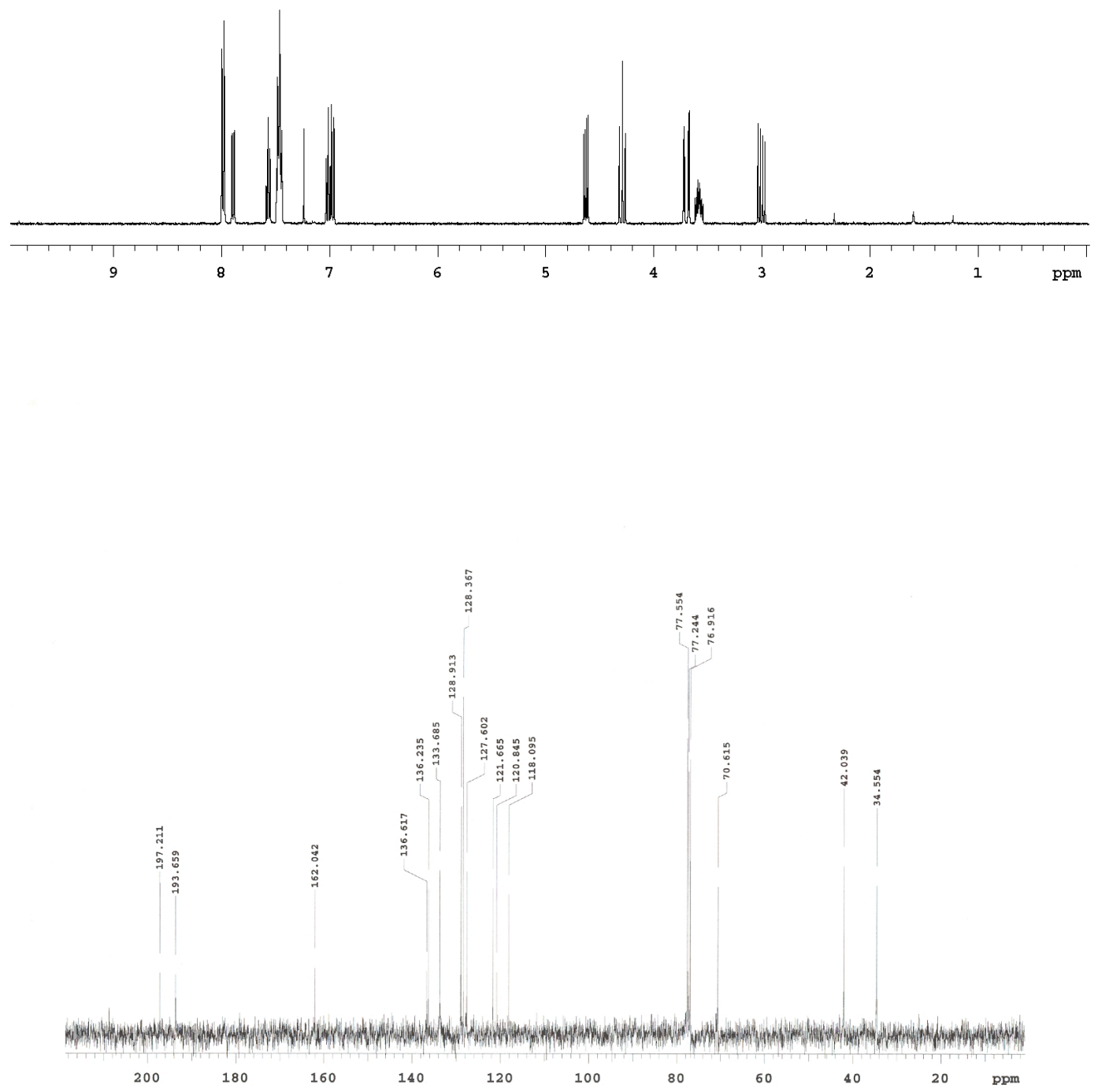
(15)

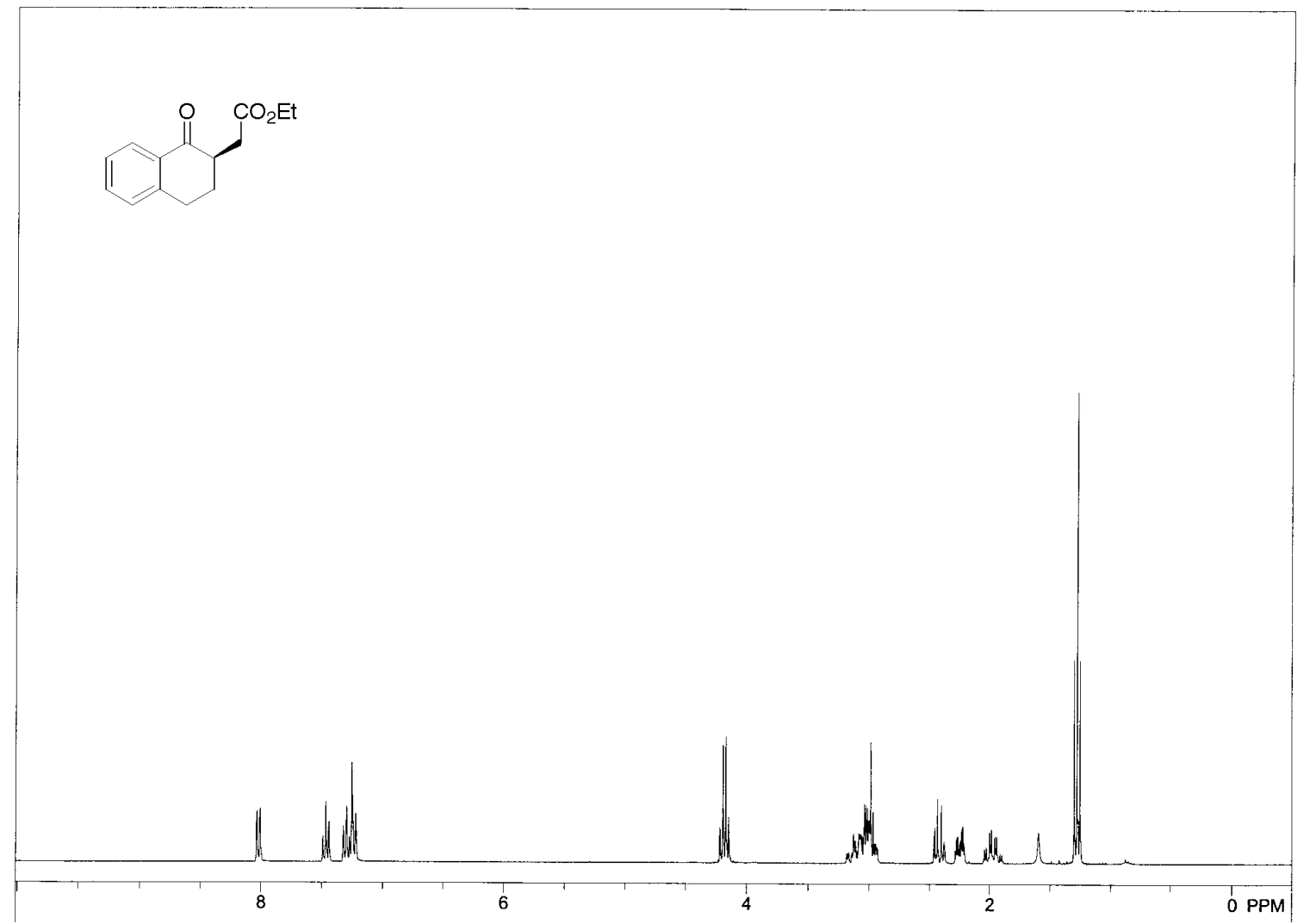


(17)

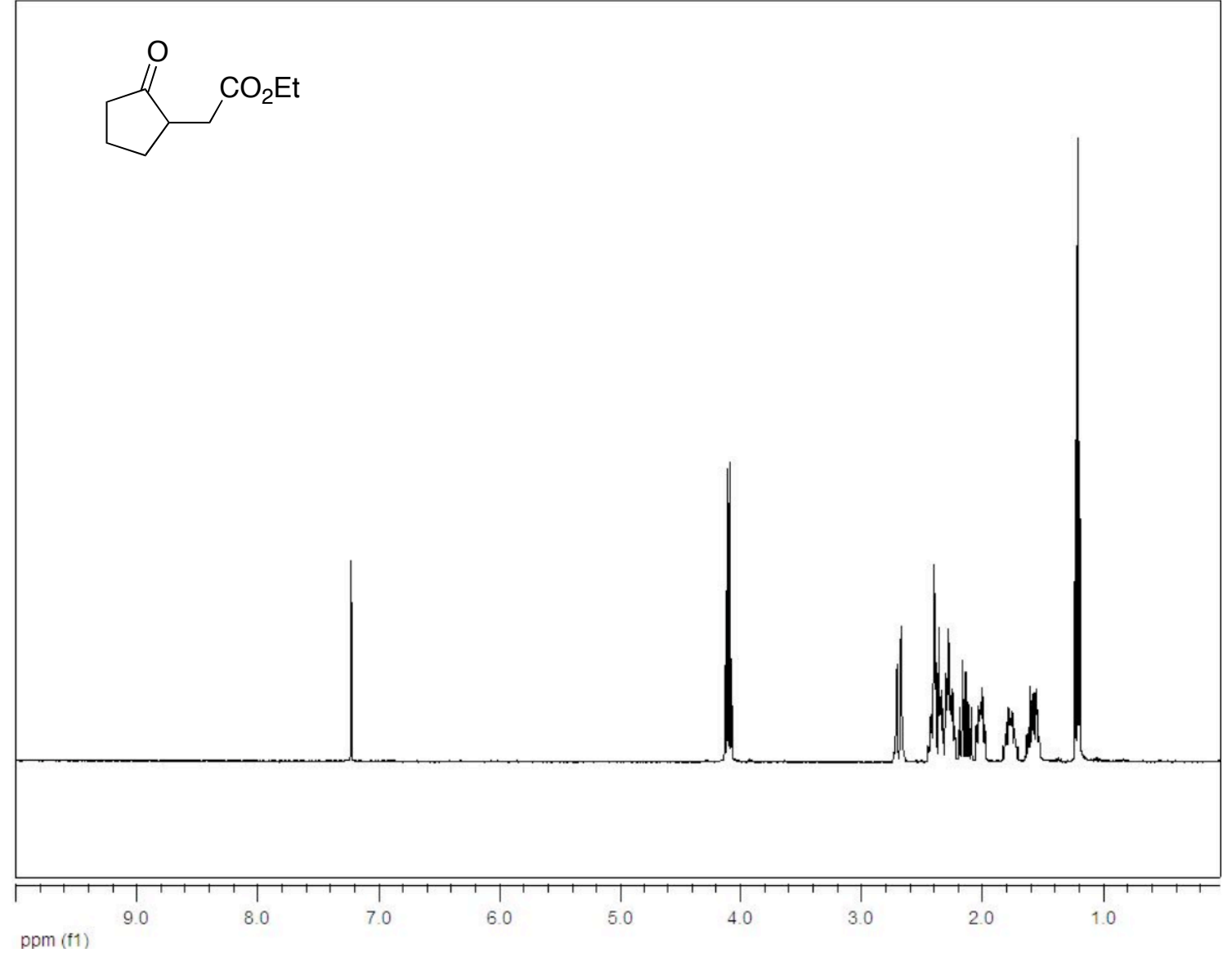


(19)
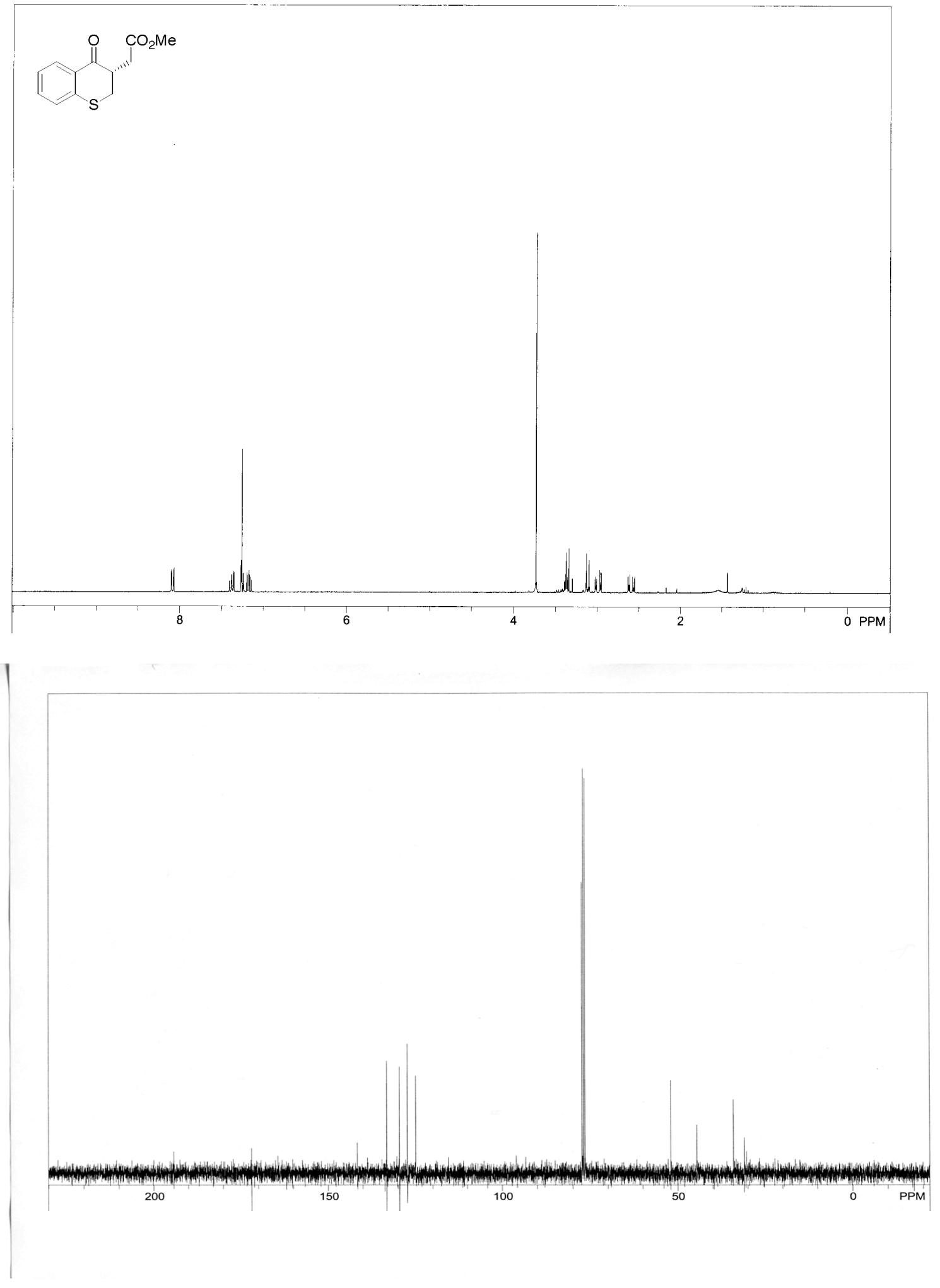

S-30 
(21)

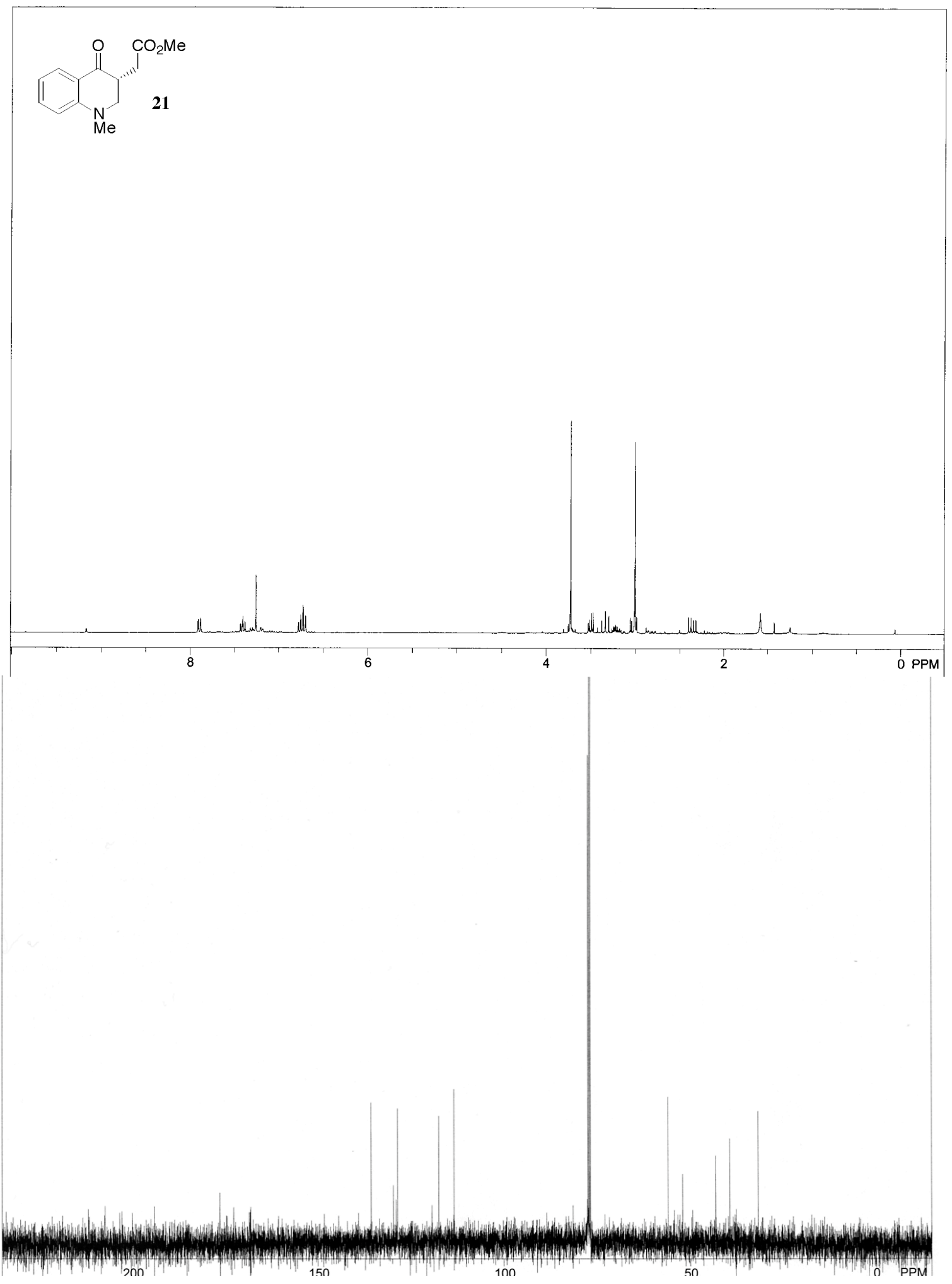


(23)
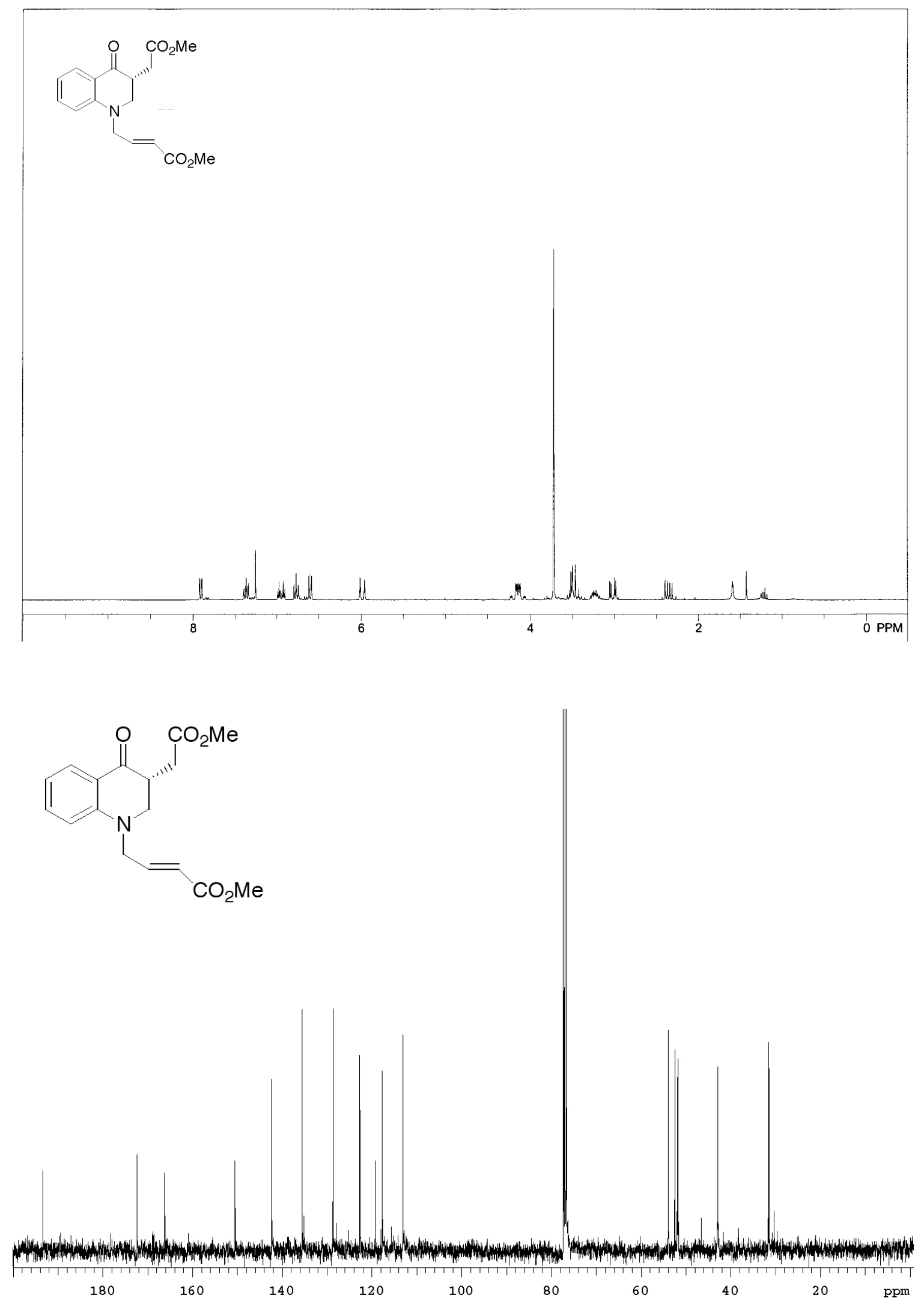


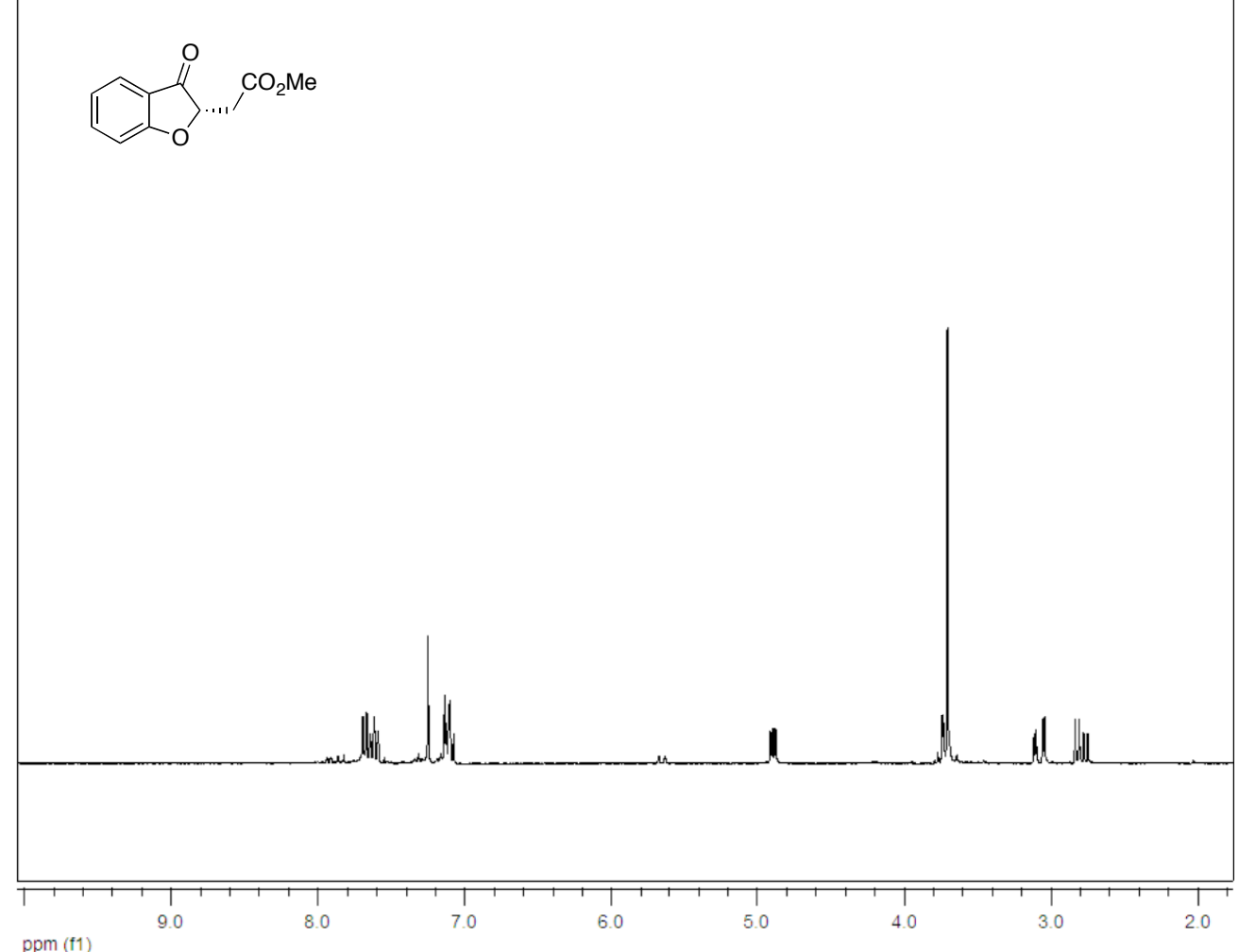

(29)

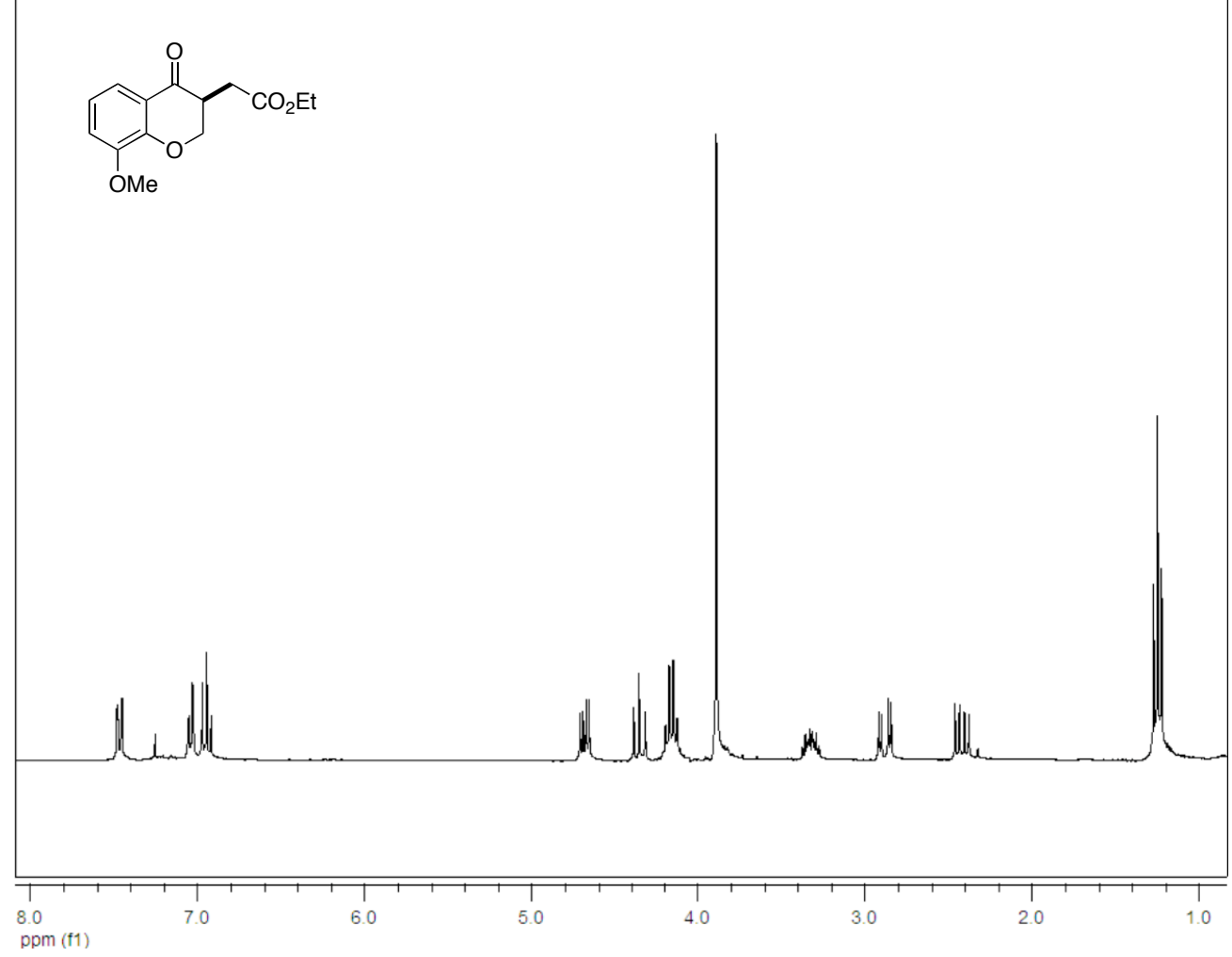


(31)

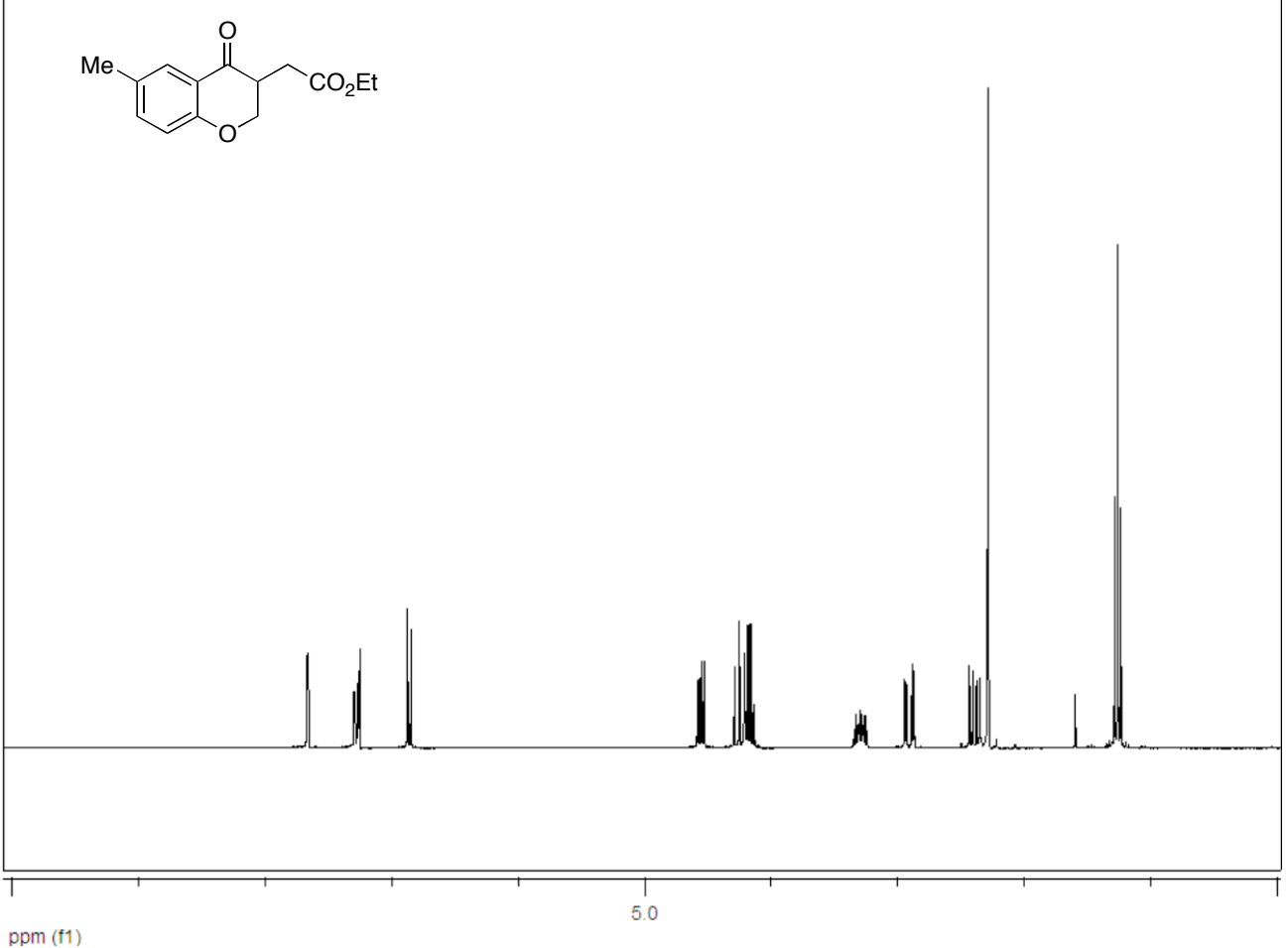


(33)
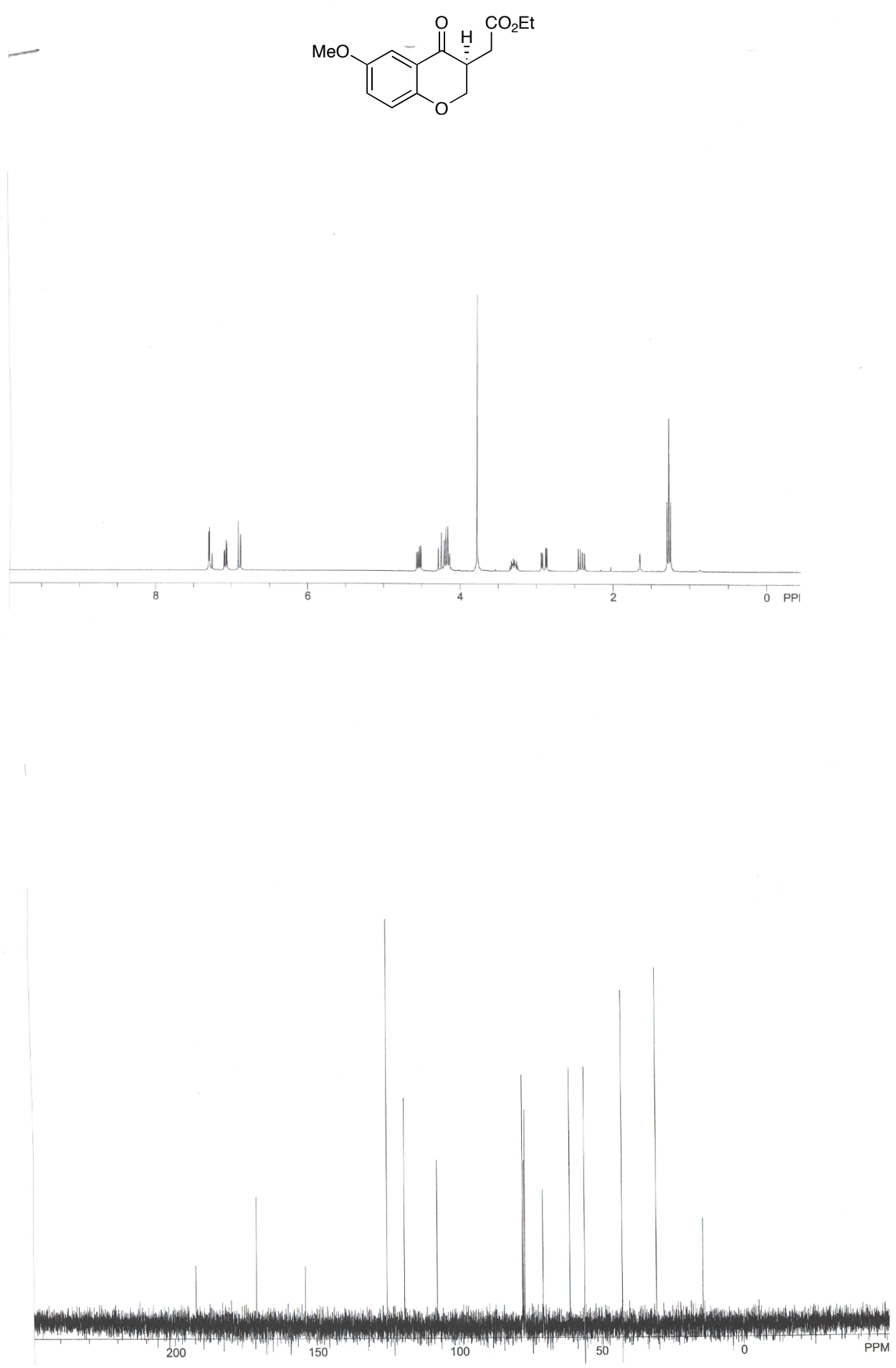
(35)
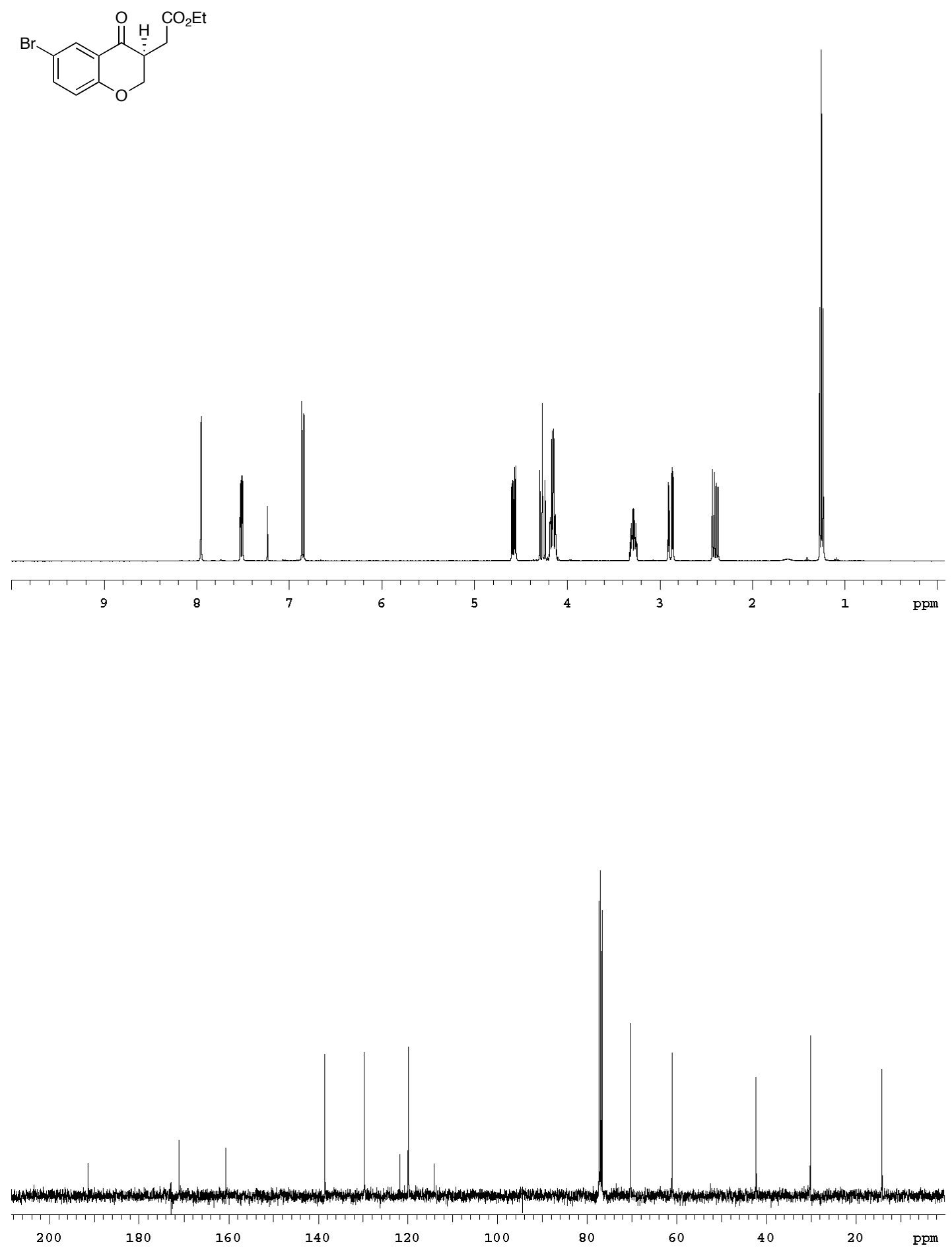

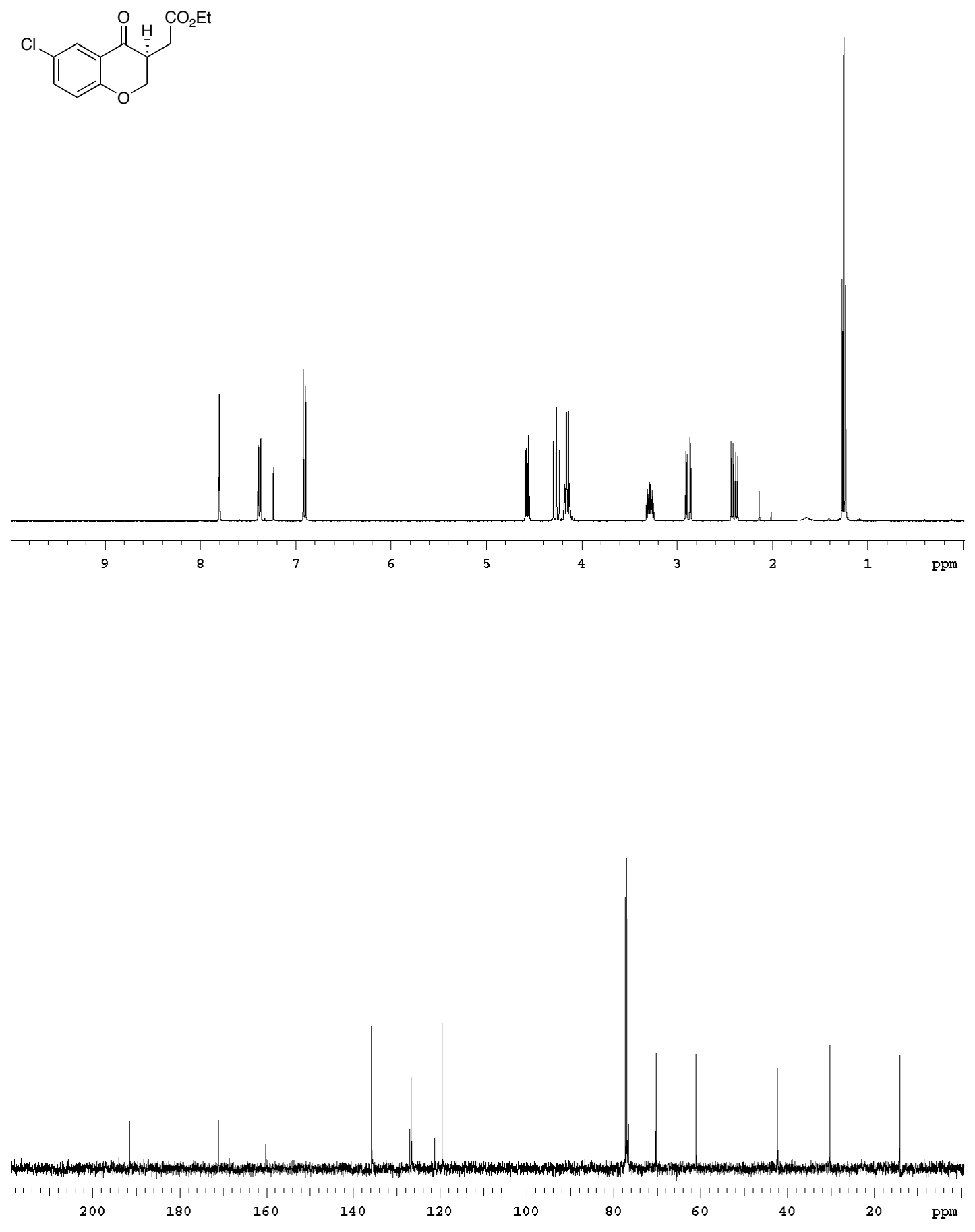

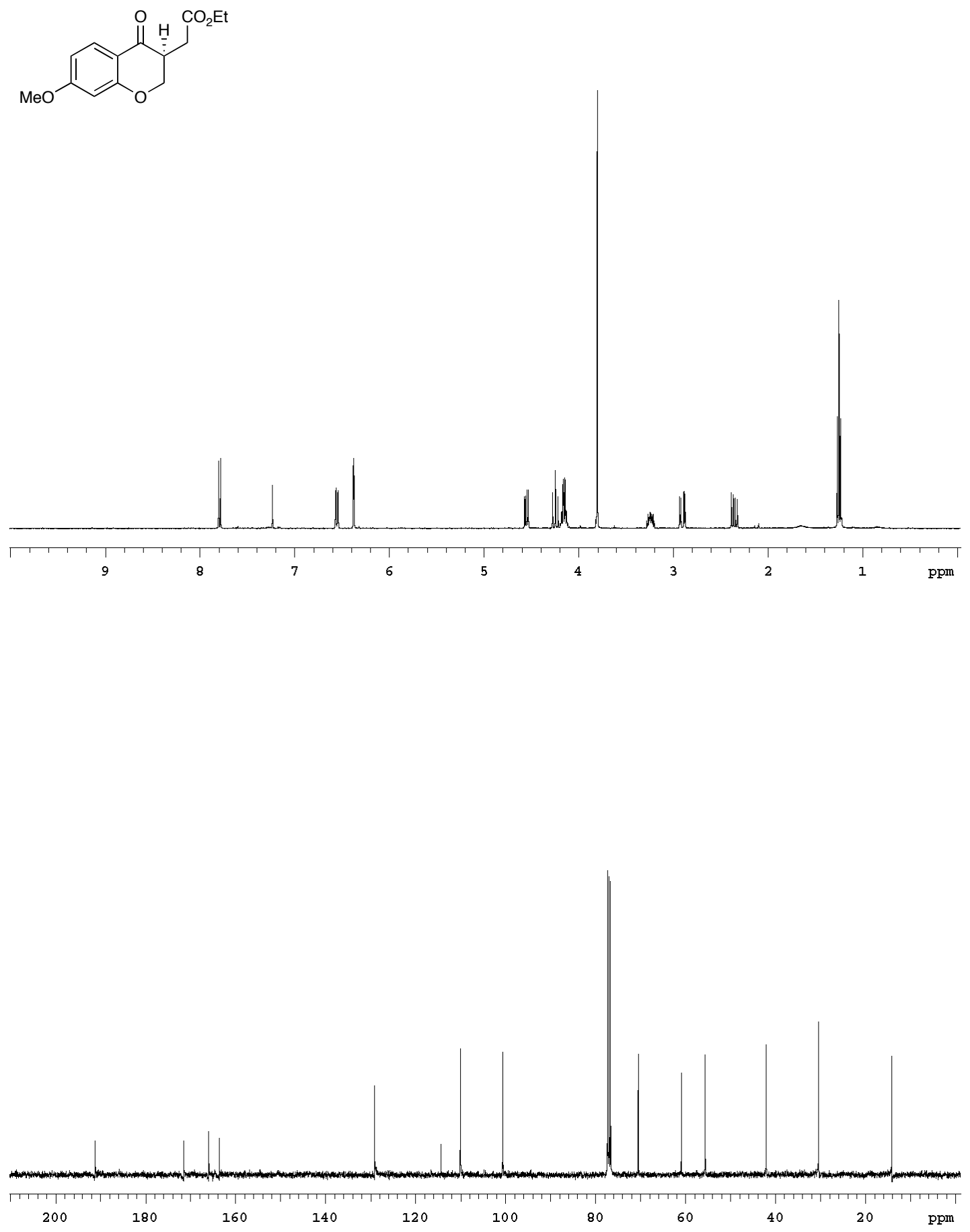
(41)<smiles>CCNc1ccc2c(c1)OCC(CC(=O)OCC)C2=O</smiles>
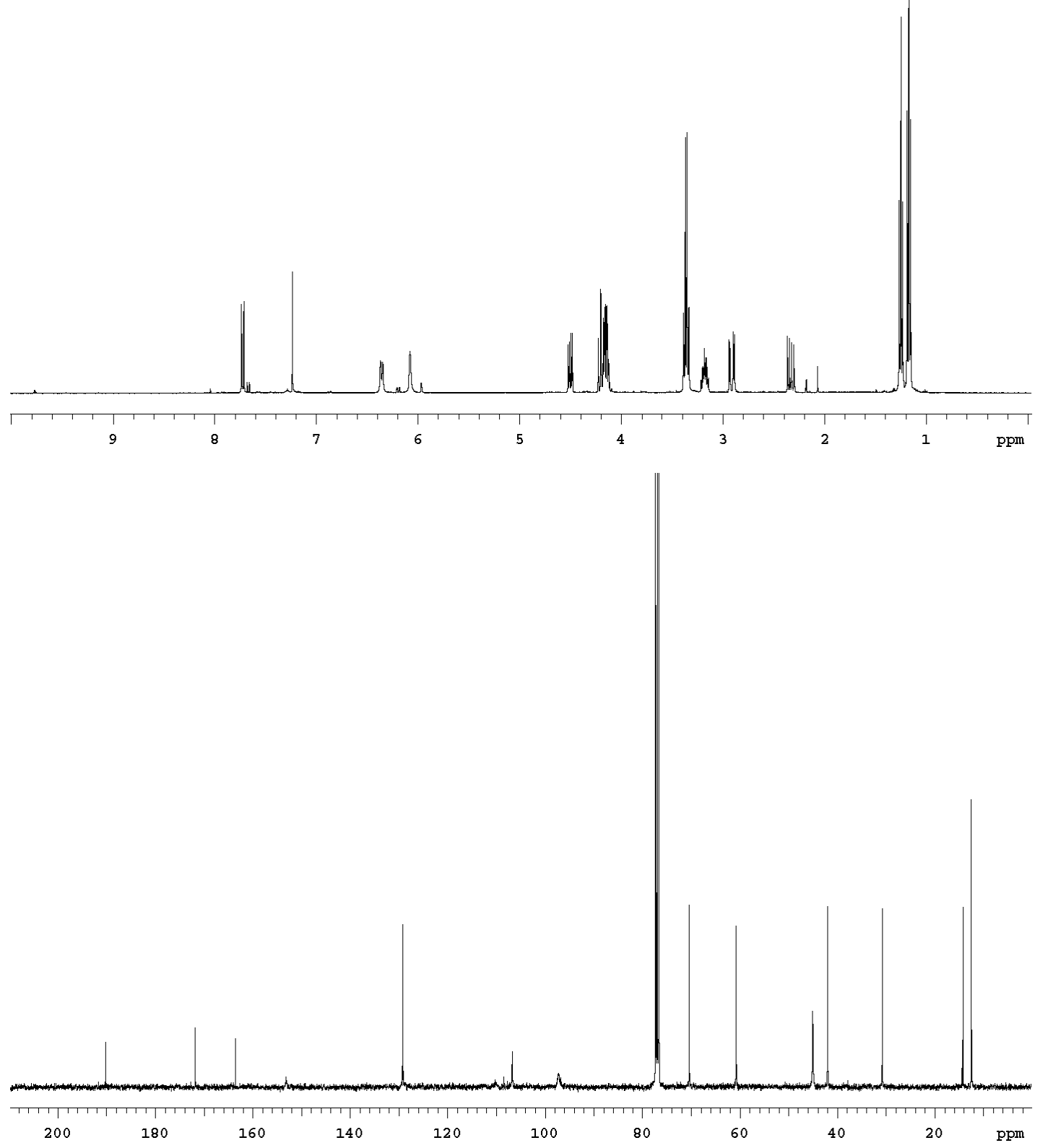
(42)

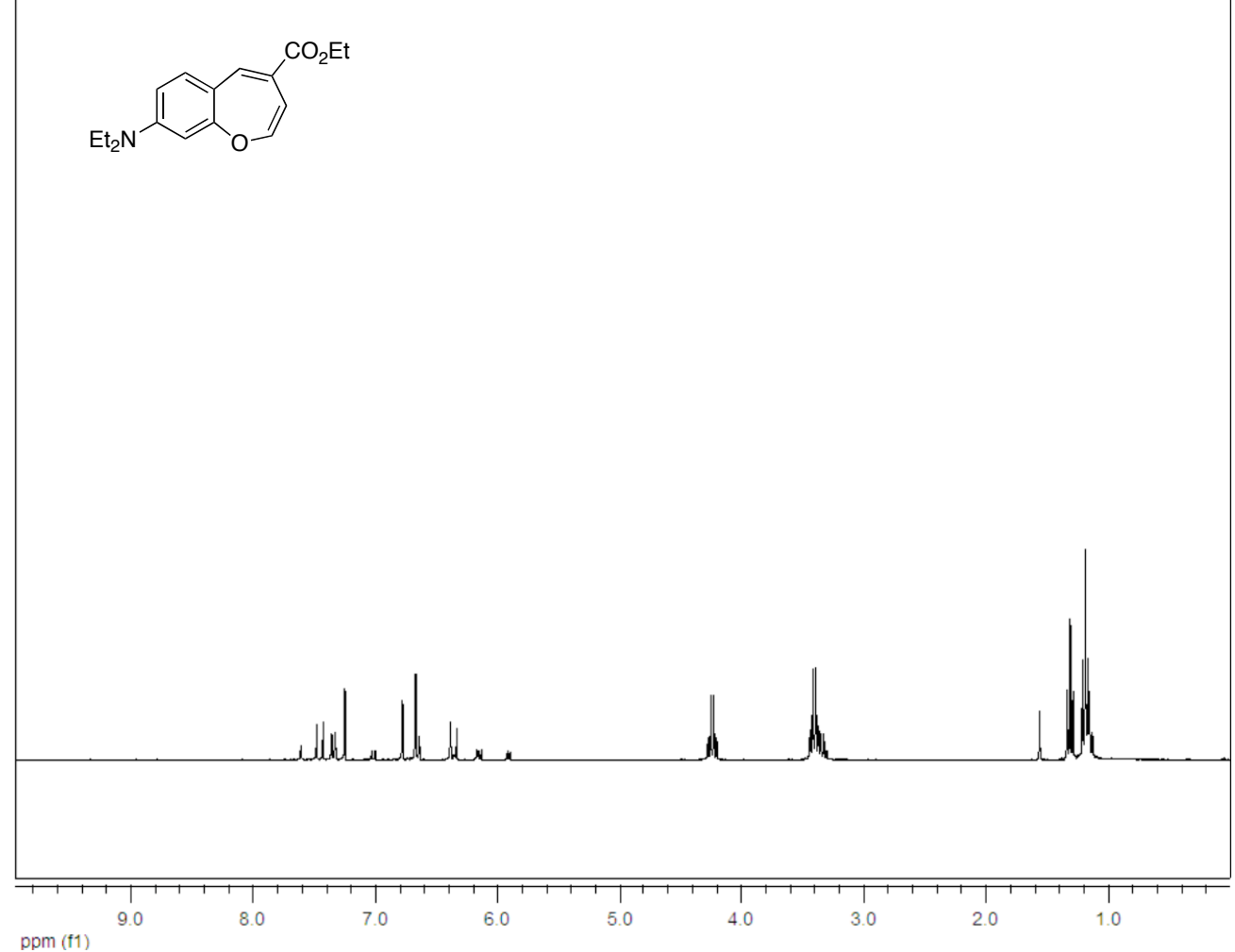


(44)
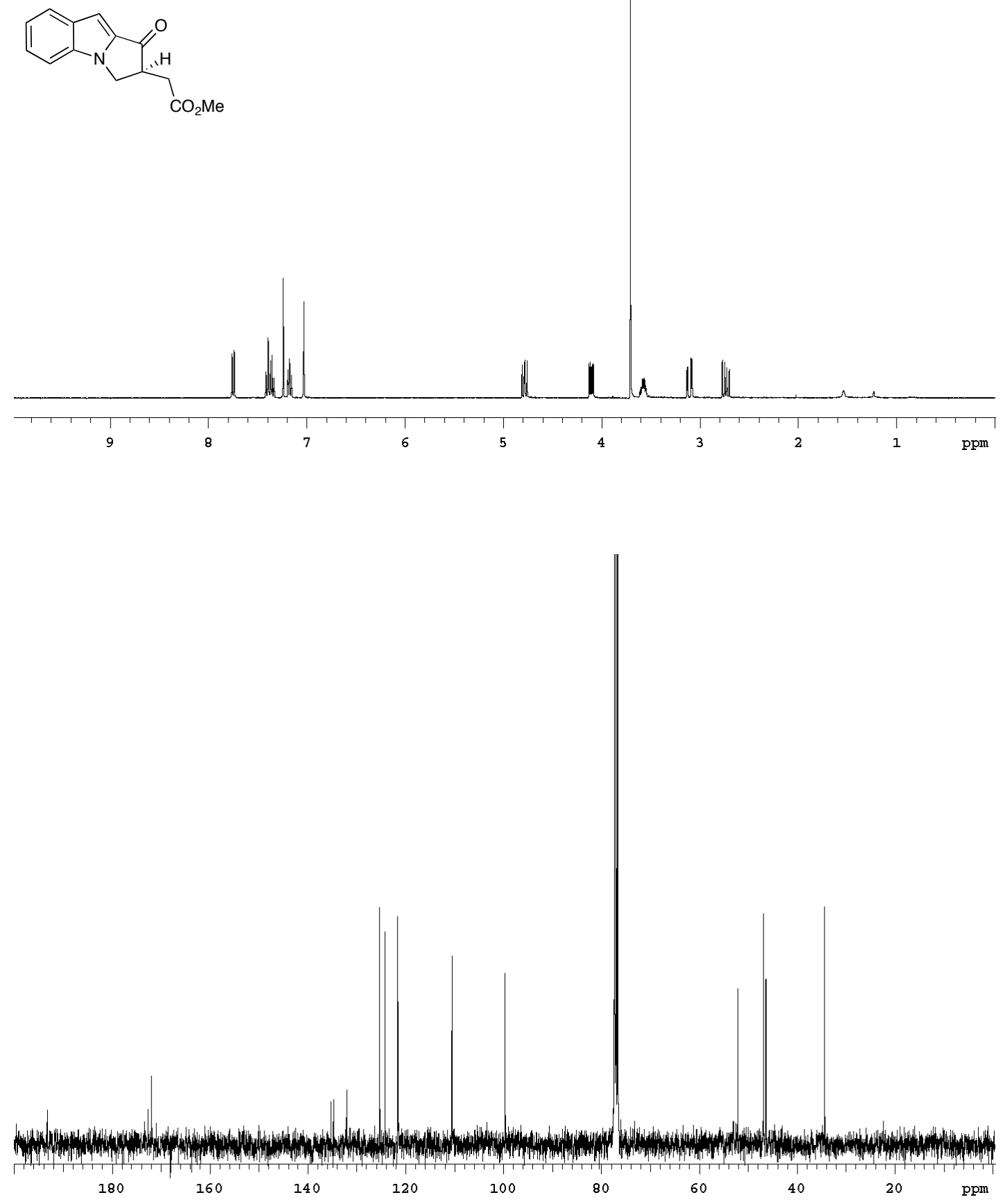

S-41 
<smiles>C=CCOC(=O)C[C@H]1COc2ccccc2C1=O</smiles>
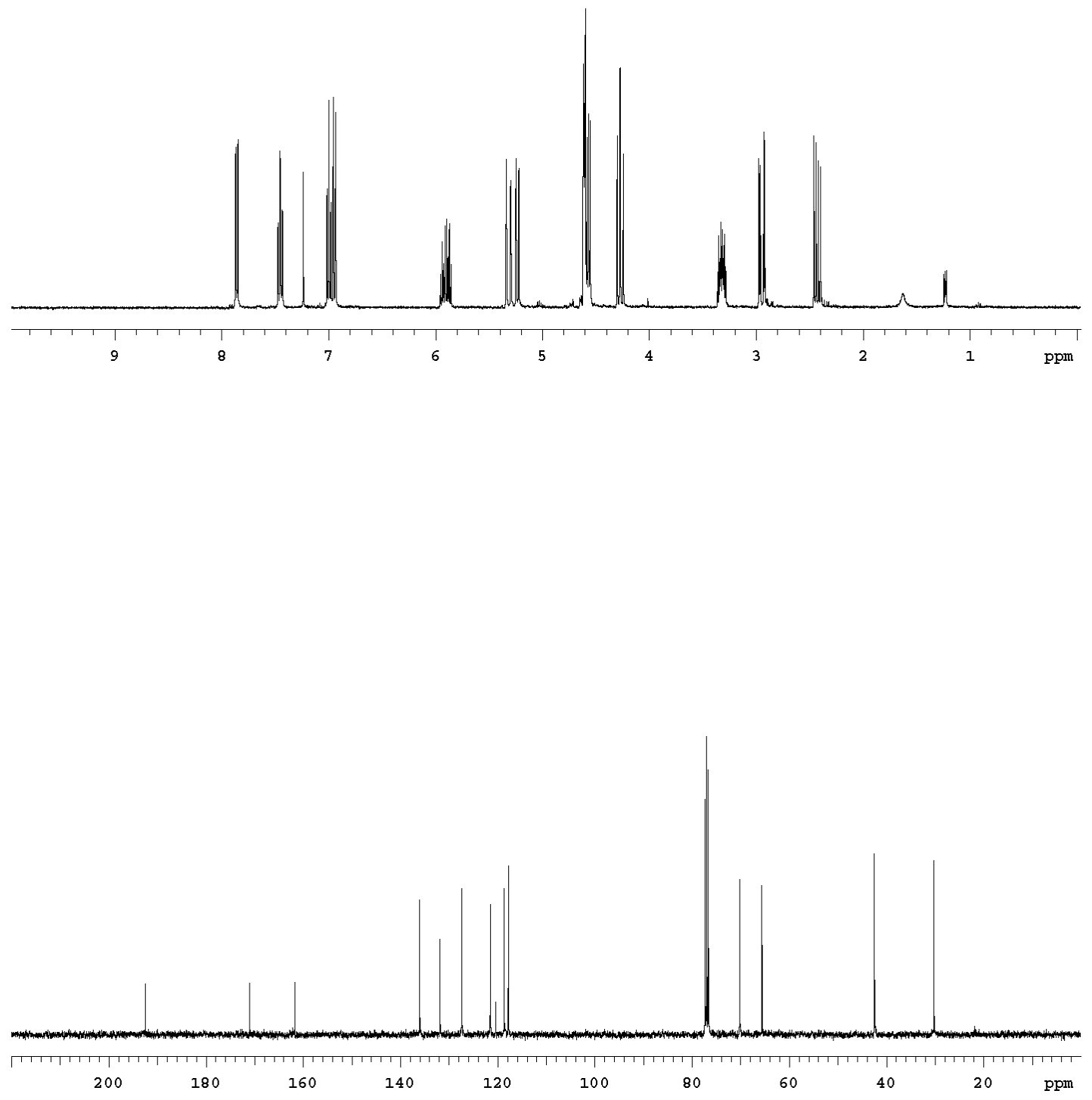
(49)
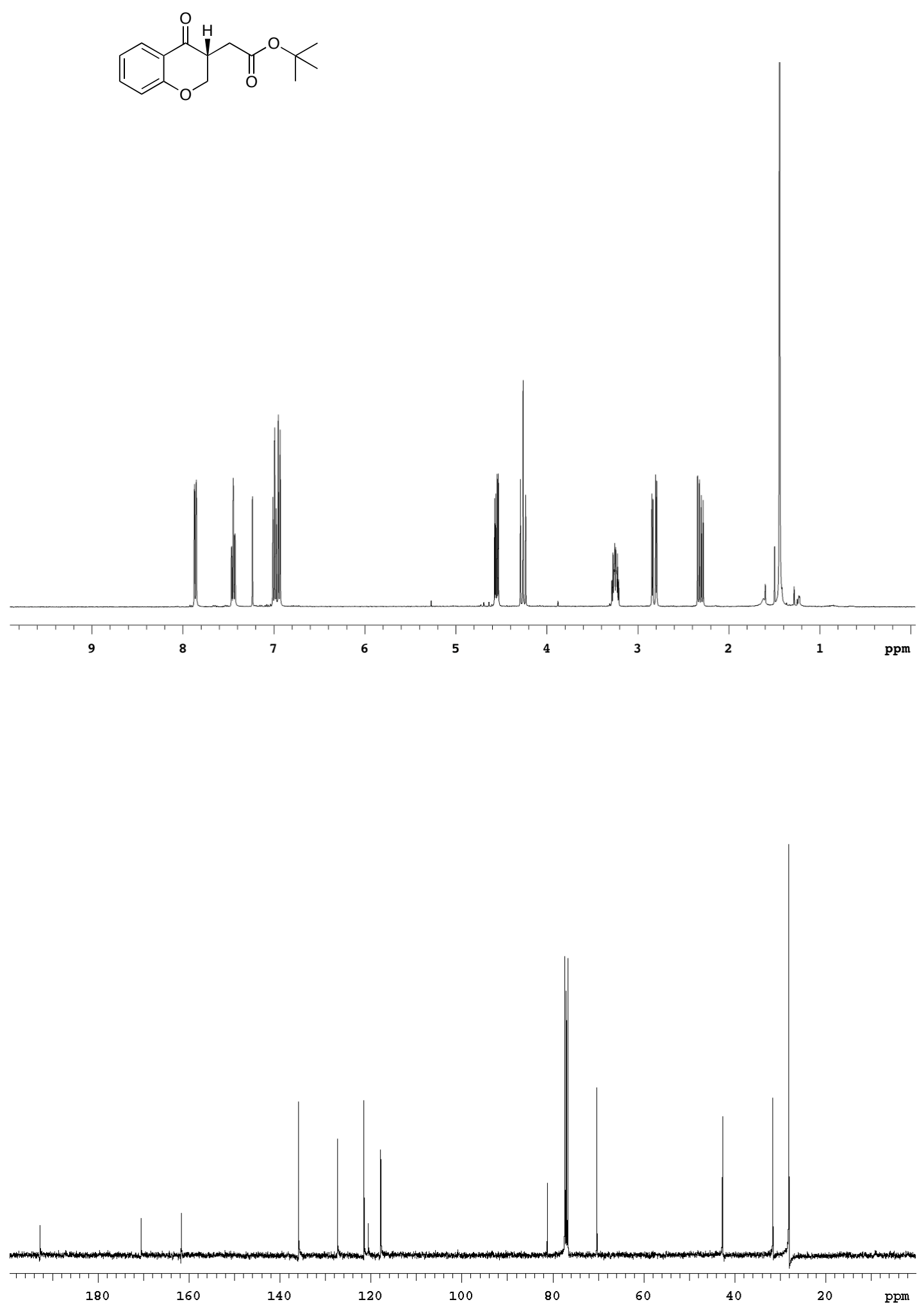
(53)<smiles>CCC(=O)C[C@H]1COc2ccccc2C1=O</smiles>
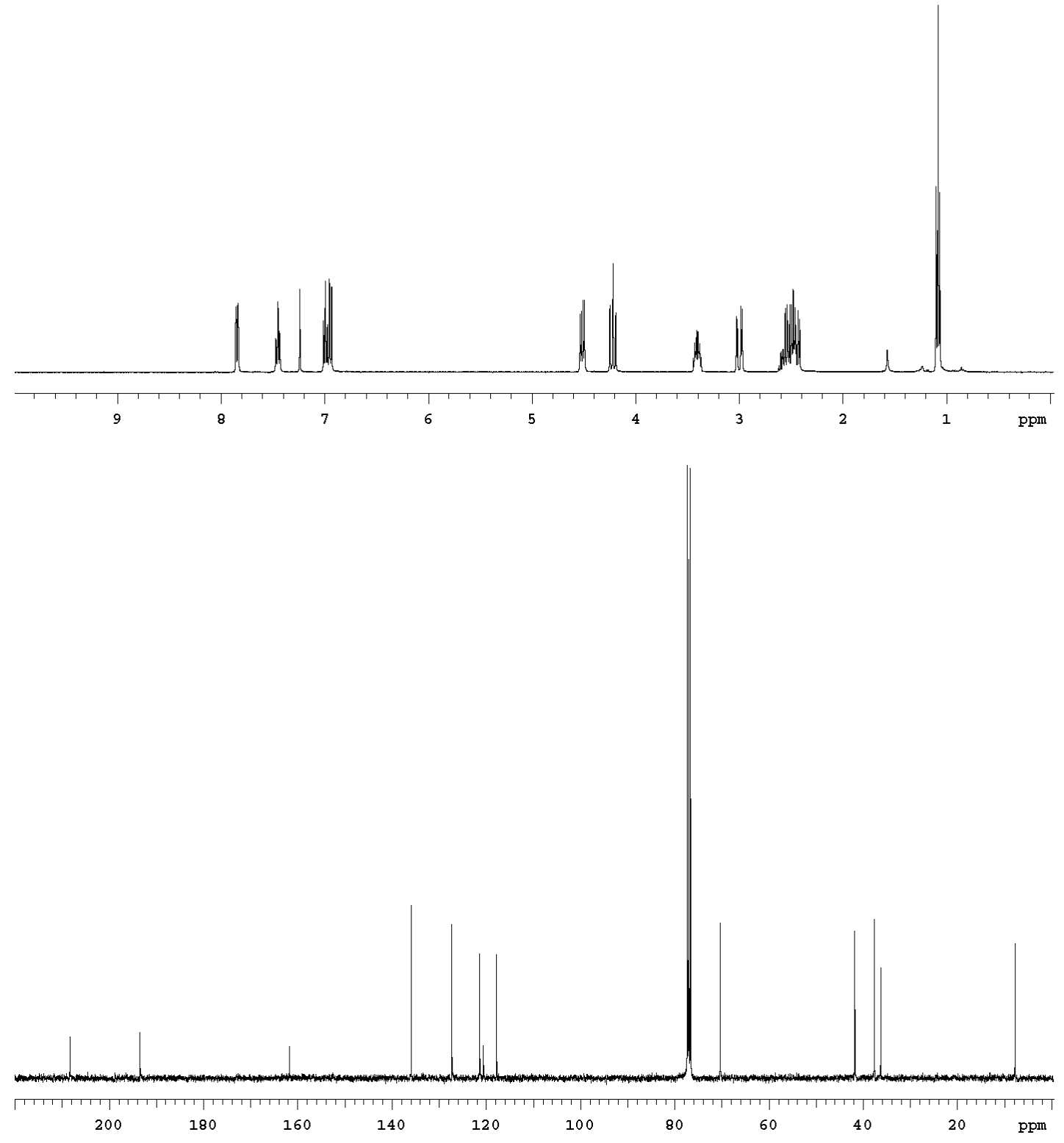
<smiles>CCOC(=O)CC1COc2ccccc2C1=O</smiles>
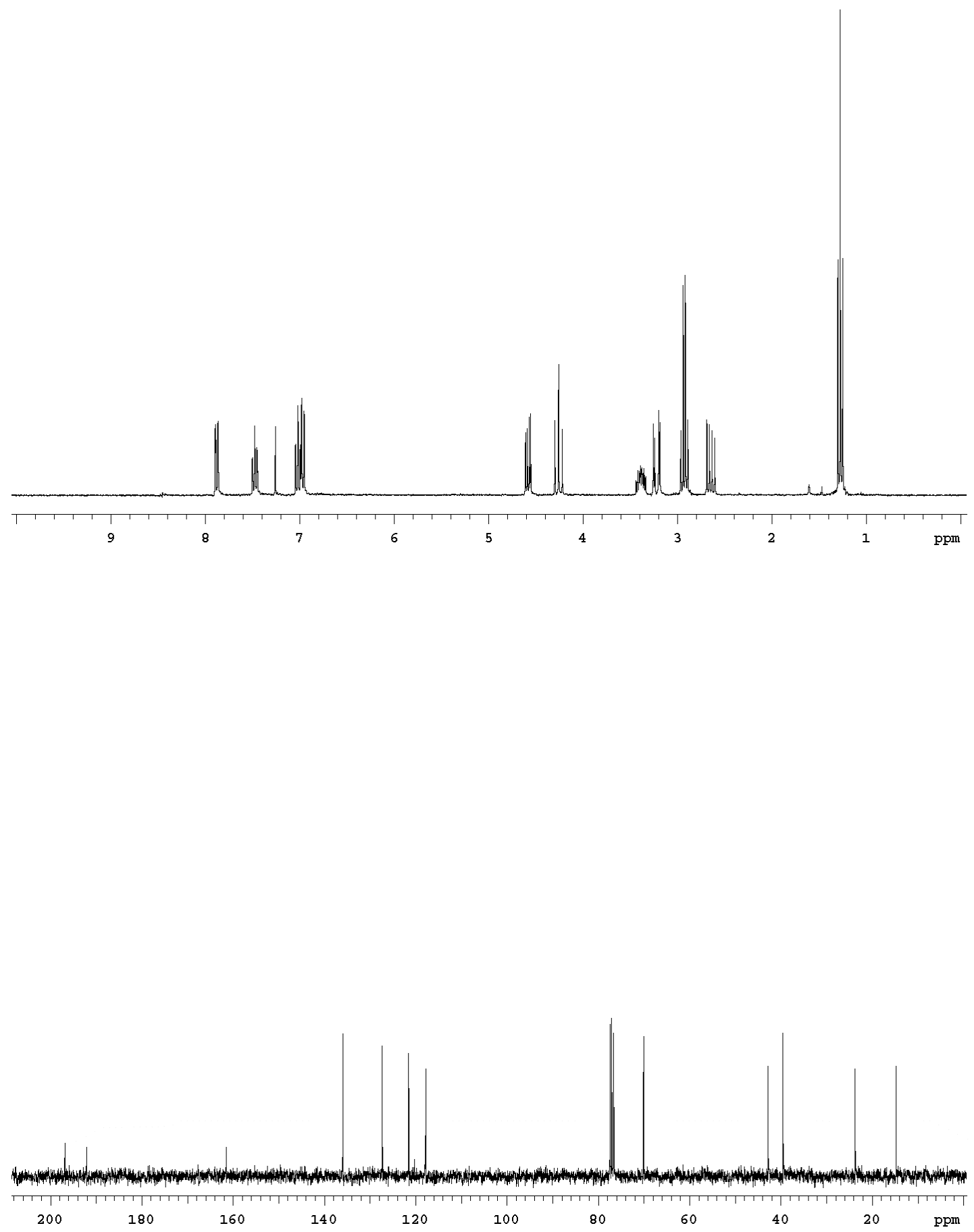

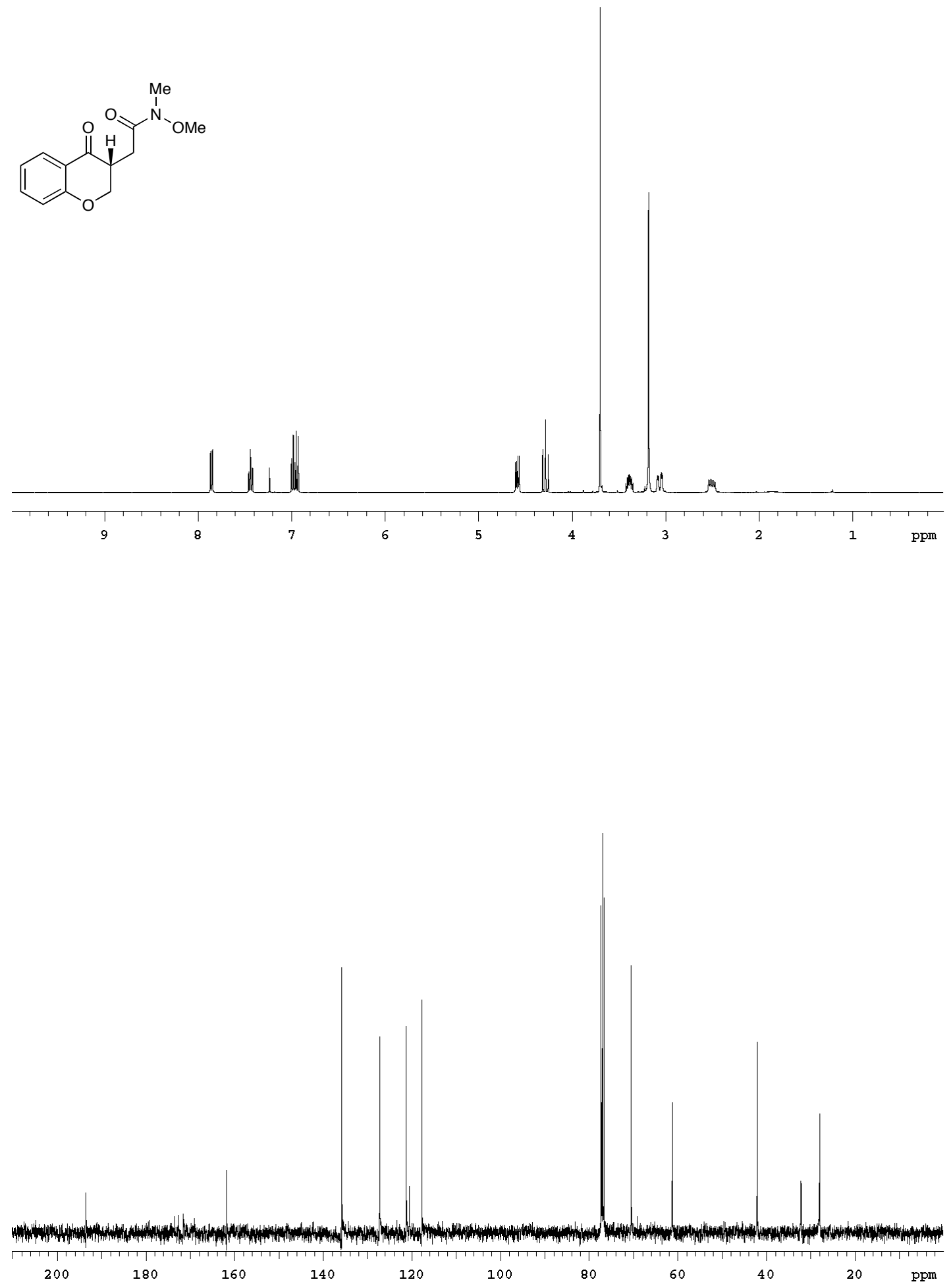
(59)<smiles>N#CCC1COc2ccccc2C1=O</smiles>
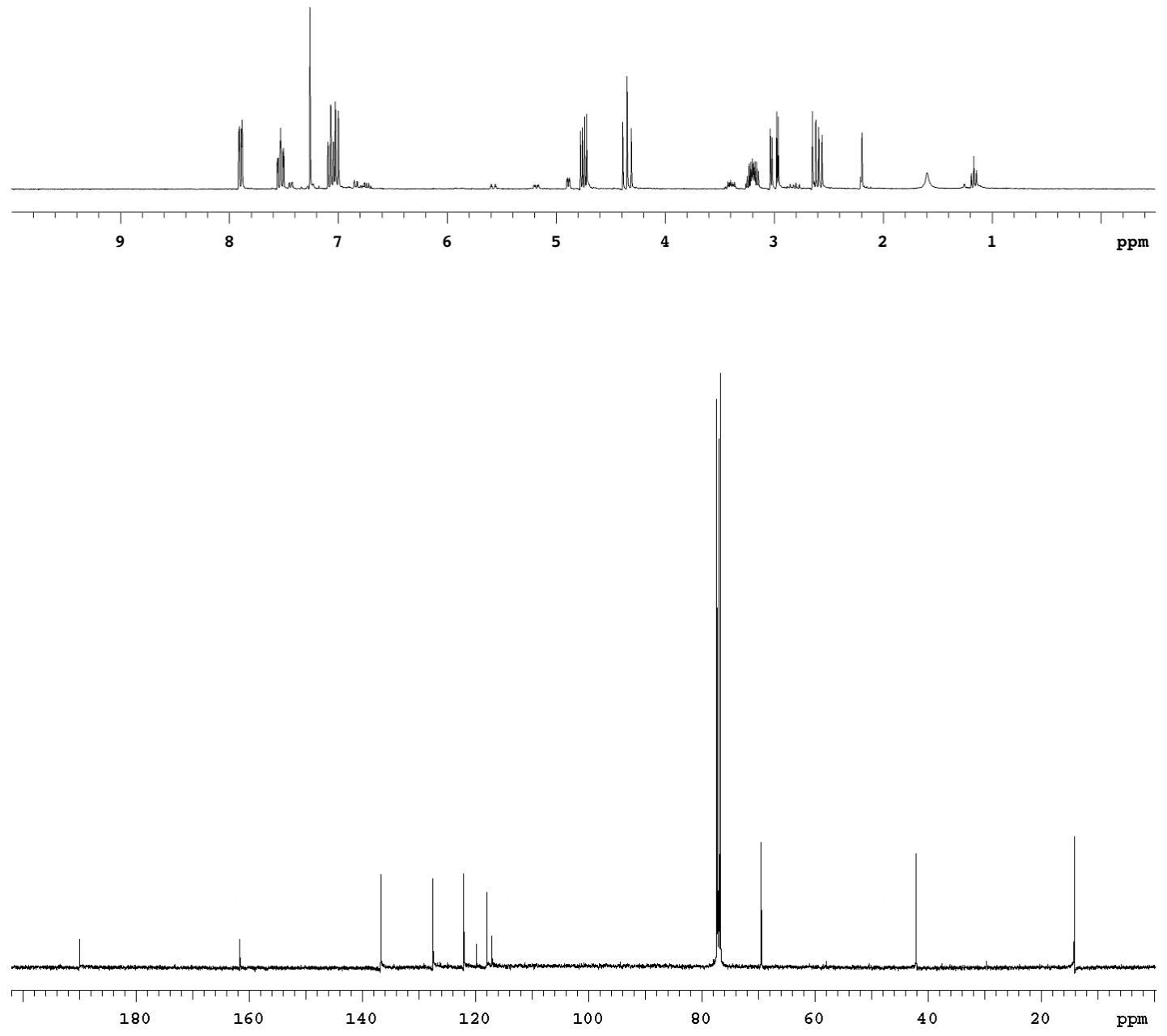

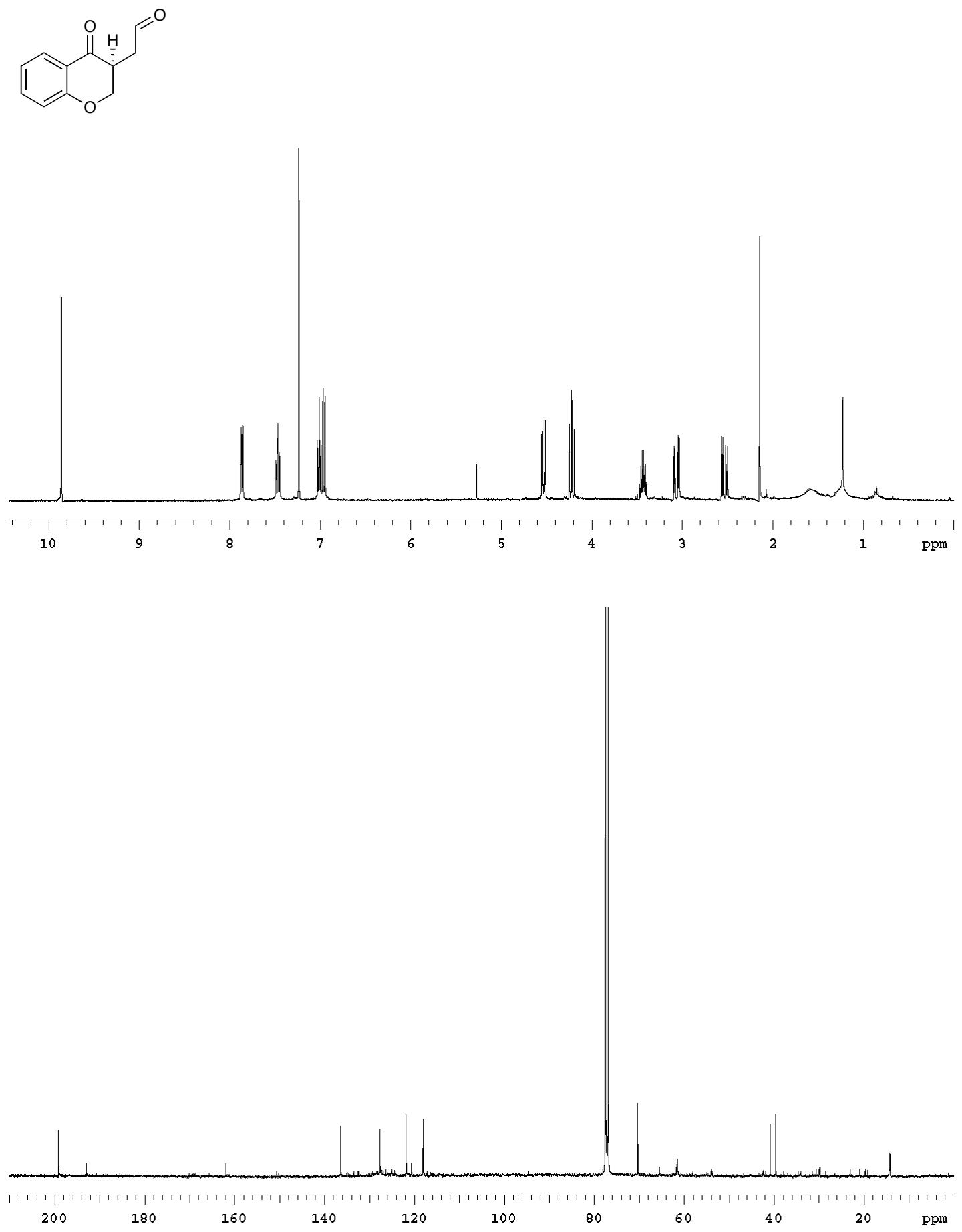
(64)
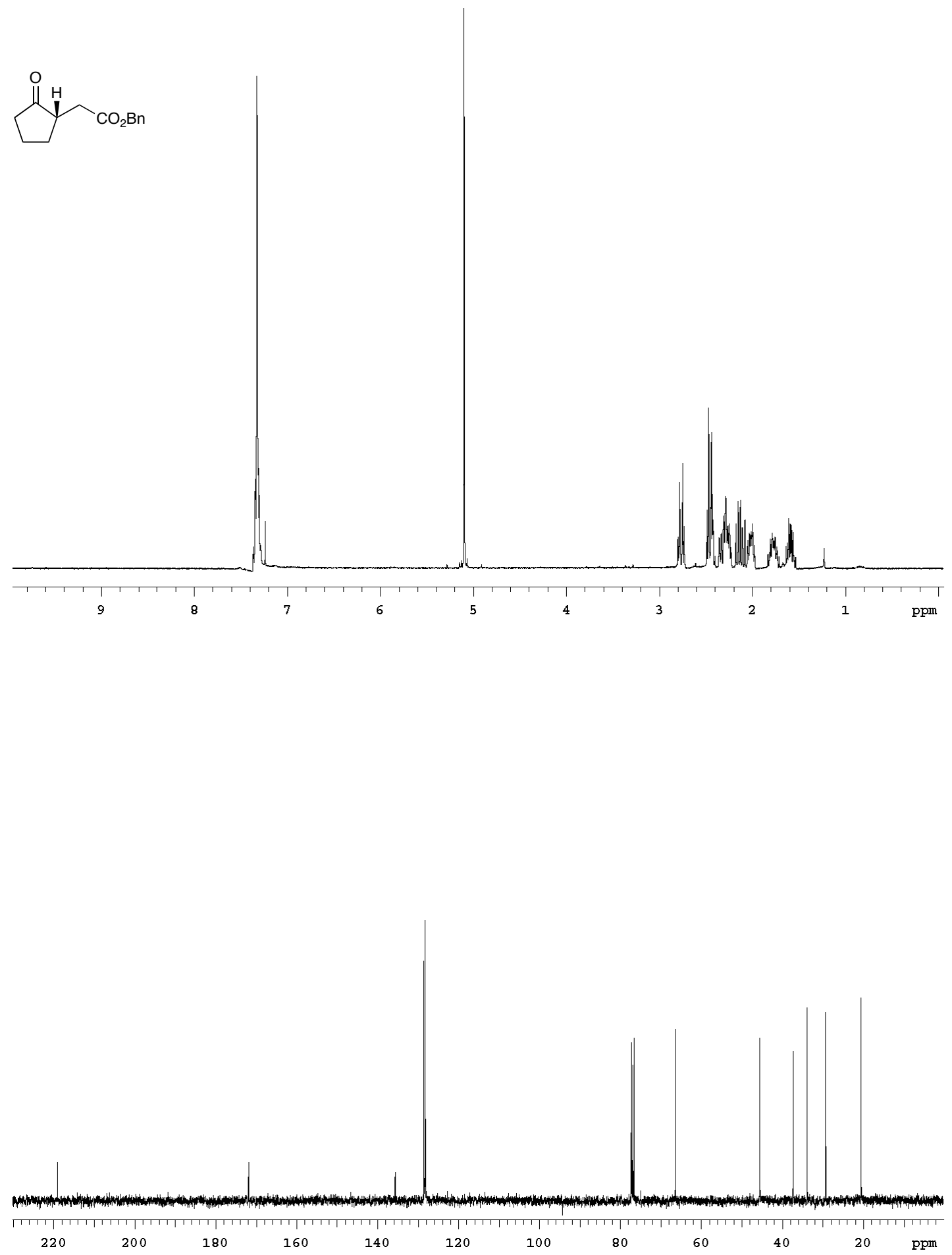
(66)<smiles>CCOCC1CN(Cl)CC1=O</smiles>
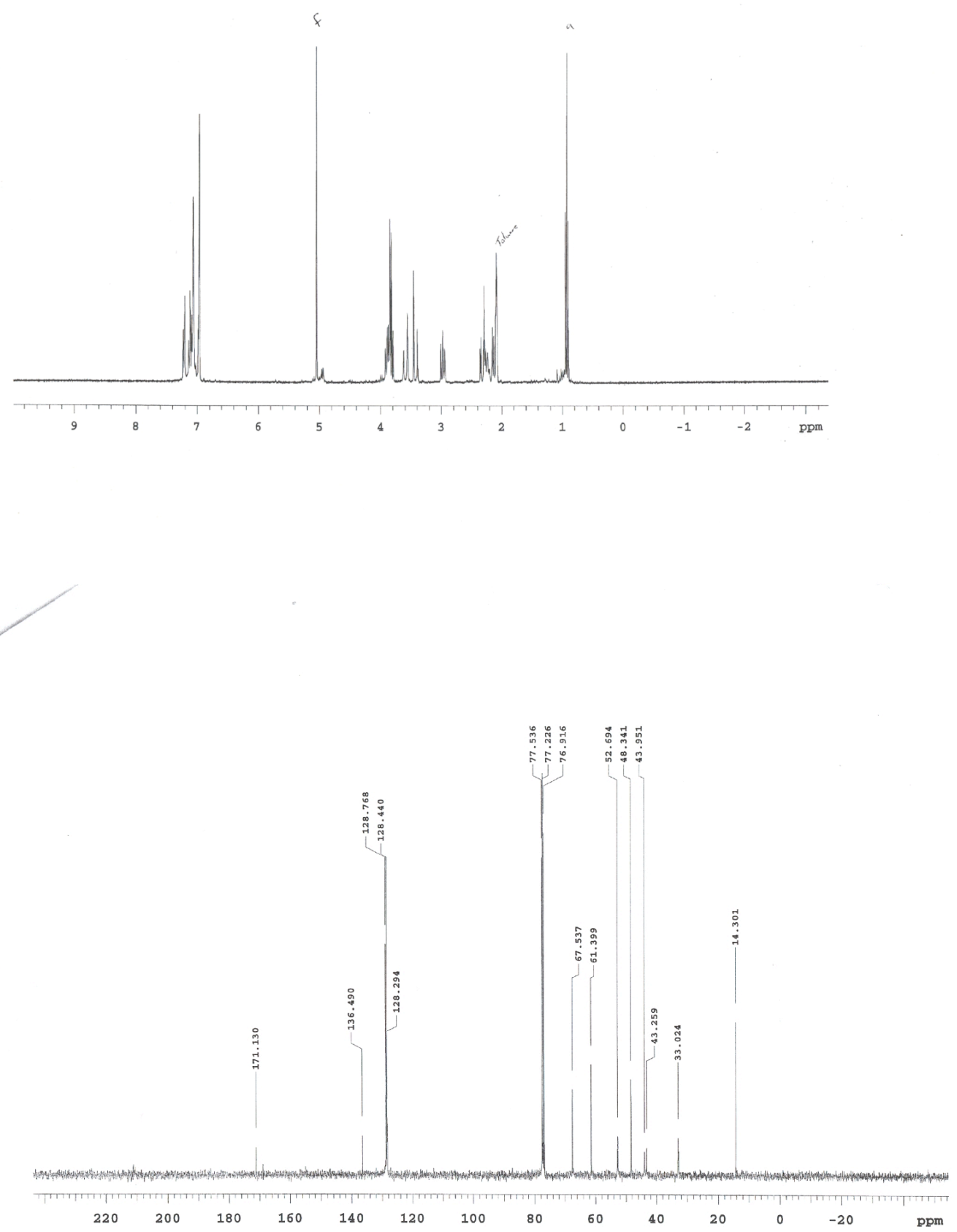
(70)

$\overbrace{\mathrm{COPh}}^{\mathrm{O}}$
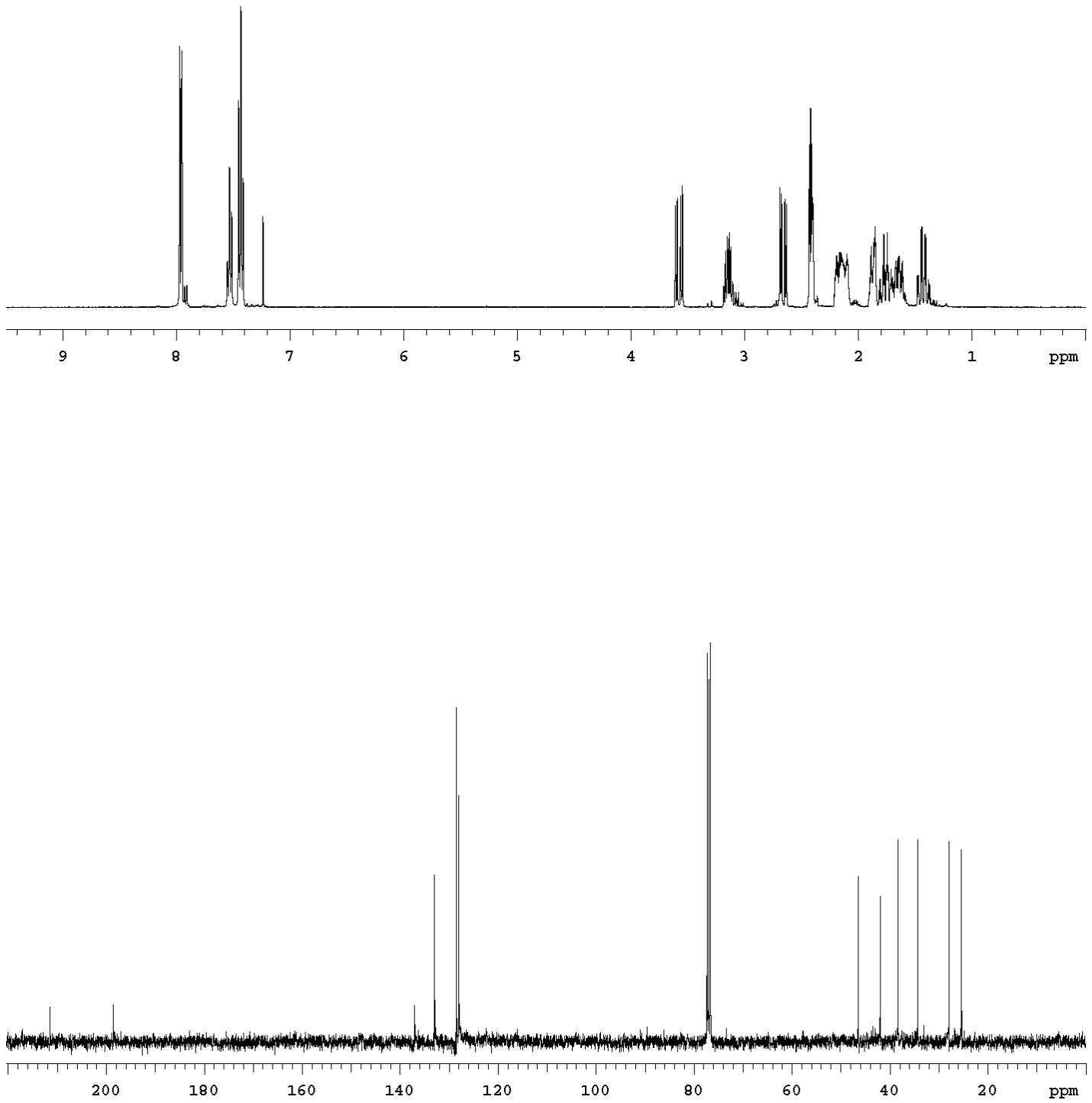

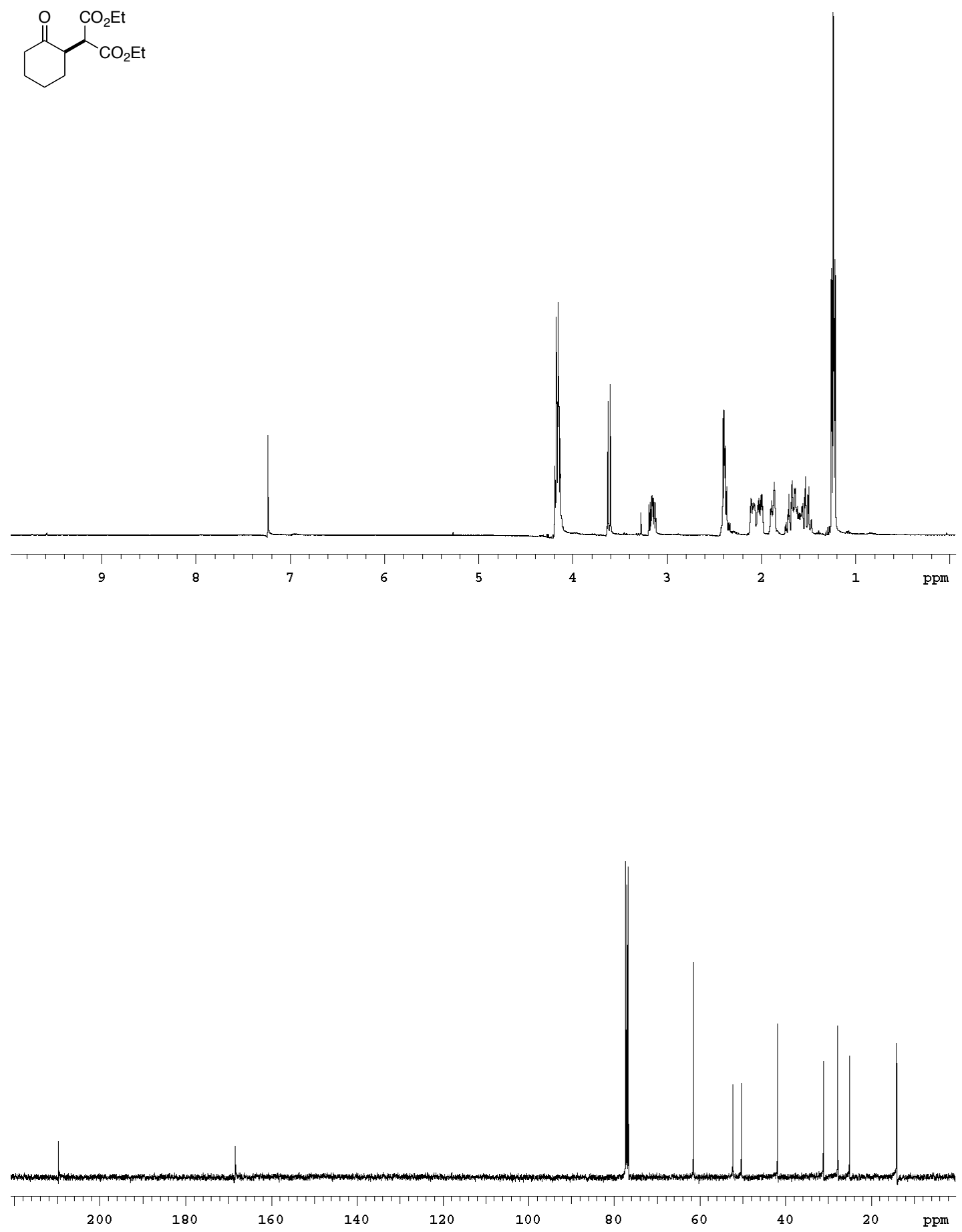


\section{References:}

${ }^{1}$ Ciganek, E. Synthesis 1995, 1311-1314.

${ }^{2}$ Kerr, M. S.; Read de Alaniz, J.; Rovis, T. J. Org. Chem. 2005, 70, 5725-5728.

${ }^{3}$ Kerr, M. S.; Read de Alaniz, J.; Rovis, T. J. Am. Chem. Soc. 2002, 124, 10298-10299.

${ }^{4}$ Enders, D.; Breuer, K.; Runsink, J. Helv. Chim. Acta. 1996, 79, 1899-1902.

${ }^{5}$ Wang, S.; Chen, G.; Kayser, M. M.; Iwaki, H.; Lau, P. C. K.; Hasegawa, Y. Can. J. Chem. 2002, 80, 613-621. 